\title{
Properties of the Photometric Components of Lenticular Galaxies
}

\author{
J. Alfonso L. Aguerri \\ Instituto de Astrofísica de Canarias, C/ Vía Láctea s/n, 38200 La Laguna, Spain \\ Correspondence should be addressed to J. Alfonso L. Aguerri, jalfonso@iac.es \\ Received 1 October 2011; Revised 12 February 2012; Accepted 31 March 2012 \\ Academic Editor: Elias Brinks
}

Copyright ( $\odot 2012$ J. Alfonso L. Aguerri. This is an open access article distributed under the Creative Commons Attribution License, which permits unrestricted use, distribution, and reproduction in any medium, provided the original work is properly cited.

\begin{abstract}
After almost one century since their definition, the origin of lenticular galaxies is still a matter of debate. Several formation processes have been proposed in order to explain the wide variety of their observational properties. These properties could indicate that the class of lenticulars is a family formed by galaxies with different formations and evolutions. Here I review the main observational properties of the photometric components of lenticular galaxies reported in recent decades. I revise the main processes proposed in order to explain their origin and evolution. I also explain the different properties of the lenticular galaxies that have evolved through each of these formation processes. A unique opportunity for understanding the origin of S0 galaxies will shortly be forthcoming. This is due to the morphological classifications of large galaxy samples that have recently been published. These classifications have given us our first ever opportunity to study large samples of lenticulars within a wide range of masses and located in a great variety of environments. These large samples will provide us with a real census of nearby lenticular galaxies and could be crucial in finally helping us to understand the origin and evolution of these galaxies.
\end{abstract}

\section{Introduction}

Lenticular galaxies were introduced by Hubble in 1936 in order to fill the gap between elliptical and spiral galaxies. Their observational properties have been analyzed for decades. Several studies have shown that lenticular galaxies exhibit a great diversity of properties, being similar to both elliptical and spiral galaxies. This makes their origin and evolution still a matter of debate, and several questions still need to be answered. Are lenticular galaxies a well-defined and homogeneous class of galaxies, or are they on the contrary a morphological class of galaxies containing galaxies with different origin and evolution?

Concerning their formation, are lenticulars formed by major mergers of galaxies, similarly to bright elliptical galaxies, or did they form through slow galaxy processes including minor satellite accretion or other secular evolution processes? Are they the final steps in the evolution of late-type galaxies due to environmental mechanisms? Some light can be shed on these questions by analyzing the different observational properties of the structural components present in lenticulars. This is the main aim of this paper. I have compiled information from the literature about the main observational properties of the photometric components of lenticulars and I review the different formation mechanisms proposed. There is a wide diversity of formation mechanisms of S0 galaxies that act on different time scales and galaxy environments. For each formation mechanism, I review the expected properties of the lenticular galaxies formed.

This paper is organized as follows. In Section 2, I have revised visual and automatic classifications of lenticular galaxies. Multicomponent surface brightness decompositions of lenticular galaxies are reviewed in Section 3. The observational properties of the structural components of S0 galaxies are given in Section 4. The special case of dwarf lenticular galaxies ( $\mathrm{dS} 0 \mathrm{~s}$ ) is reviewed in Section 5. The different formation mechanisms of S0 galaxies are described in Section 6. Finally, some conclusions and future perspectives are given in Section 7.

\section{Classification of Lenticular Galaxies: Visual versus Automatic}

2.1. Visual Galaxy Classification: Early Definitions of Lenticular Galaxies. Galaxy classification is a visual task. Several visual classifications have been proposed since the discovery of galaxies (extragalactic nebulae) at the beginning of the 


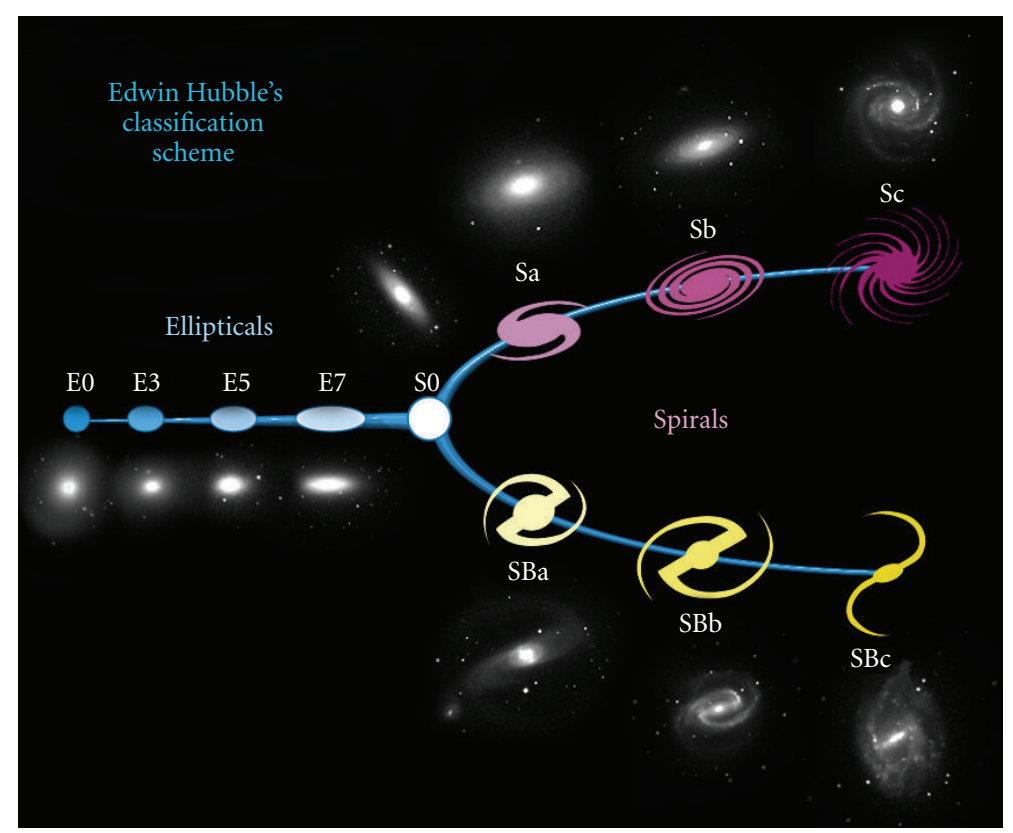

FIgURE 1: The Hubble tuning fork diagram. A schematic view of the Hubble galaxy classification. Credit: NASA and ESA.

20th century (see $[1,2]$ for good reviews on galaxy classifications). One of the pioneering classification systems was proposed by Reynolds [3]. He classified the spiral galaxies within a sequence of shapes that he defined as classes I to VII. Six years later, Hubble published the first version of his famous classification scheme. Galaxies were divided in two main classes: ellipticals (Es) and spirals. The elliptical galaxies were located in seven groups according to their flatness from E0 (round Es) to E7 (the most flattened Es). In addition, the spiral galaxies were put into adjacent classification groups depending on the bulge and the spiral arm relationships ( $\mathrm{Sa}$, $\mathrm{Sb}$, and Sc groups). Hubble established a sequence of shapes from E0 to Sc with continuity between E7 and Sa galaxies [4]. A decade later, Hubble published a new version of his classification system and introduced the type S0 (also called lenticulars by de Vaucouleurs [5]) to fill the gap between E7 and Sa galaxies [6]. Lenticular galaxies were thus those showing a prominent central spheroidal component, similar to Es, but also a disc-like structure, similar to spirals, but without spiral arms. The properties of S0 galaxies were later updated in several works (see [7-9]). The Hubble classification is usually represented in a schematic way by the so-called Hubble tuning fork diagram (see Figure 1)

Several galaxy catalogues show visual classifications of galaxies with a high level of detail. These collections contain examples of the different morphological types of galaxies, including lenticulars, and give the classification criteria for each galaxy type. Among the earlier generations of catalogues, we may mention the Hubble Atlas [10], the Revised Shapley-Ames Catalog [8], and the de Vaucouleurs Atlas $[11,12]$. Although the definition of S0 galaxies is well established in these catalogues, visual differentiation between Es and S0s is not an easy task. Unless typical discs structures such as bars or dust lanes are present in the photometrical images. Figure 2 shows examples of four nearby S0 and E galaxies. In this case, the two lenticular galaxies show strong dust lanes. In several papers, van den Bergh has argued that not all S0 galaxies are properly classified as intermediate between E and Sa galaxies (see, e.g., [13-16]). He found that the frequency distribution of galaxy luminosities for S0 galaxies is not intermediate between $\mathrm{E}$ and Sa objects. Lenticulars are about one magnitude fainter than E and Sa galaxies. He proposed that S0 galaxies are a class of galaxies that includes different types of objects that only have a morphological similarity. The misclassification of E and S0 galaxies has been also pointed out in several recent papers from the SAURON and ATLAS-3D groups (e.g., [17-19]), who have studied the 2D kinematics of a large sample of nearby galaxies visually classified as Es and S0s. van den Bergh [20] even proposed a new classification system of galaxies in which spirals and lenticulars formed parallel sequences. This has recently been shown by different groups of researchers (see, e.g., [18, 21, 22]).

These studies indicate how difficult the classification of $\mathrm{E}$ and S0 galaxies is and how difficult it is to select galaxy samples containing only E or S0 galaxies using only photometric information (see [23] and references therein). Fortunately, the number of large samples of S0 galaxies including morphological and/or kinematical properties have increased during the last decade. I mention here the recent near-IR S0galaxy atlas of S0 galaxies (NIRSOS) by Laurikainen et al. (see [24]). It includes detailed morphological information of a sample of 185 S0 galaxies. The SAURON and the ATLAS-3D projects (e.g., [17-19]) have also provided detailed kinematic studies of large samples of Es and S0 galaxies, which have been used for a new classification of early-type galaxies according to their kinematical properties. Figure 3 shows the new classification scheme proposed by the ATLAS ${ }^{3 \mathrm{D}}$ project. In this classification lenticular galaxies are fast rotators that form a parallel sequence to spiral galaxies. 


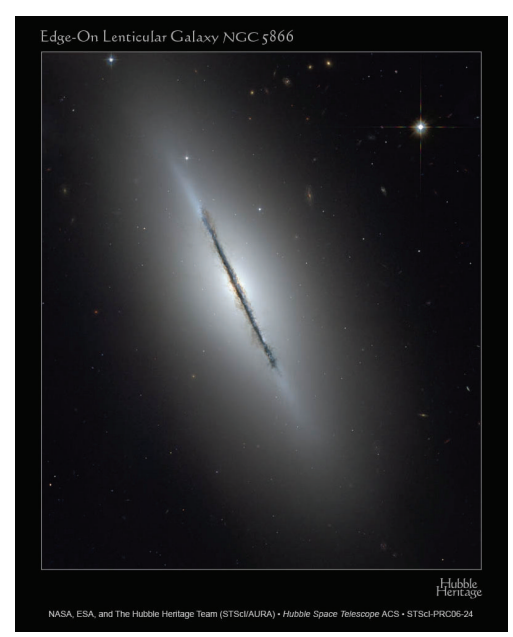

(a)

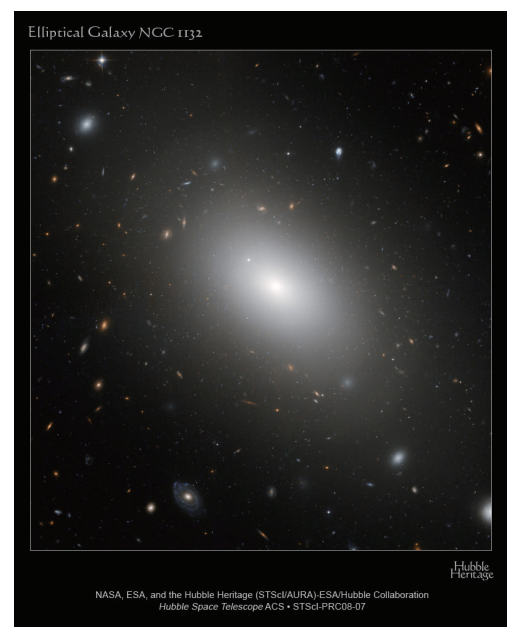

(c)

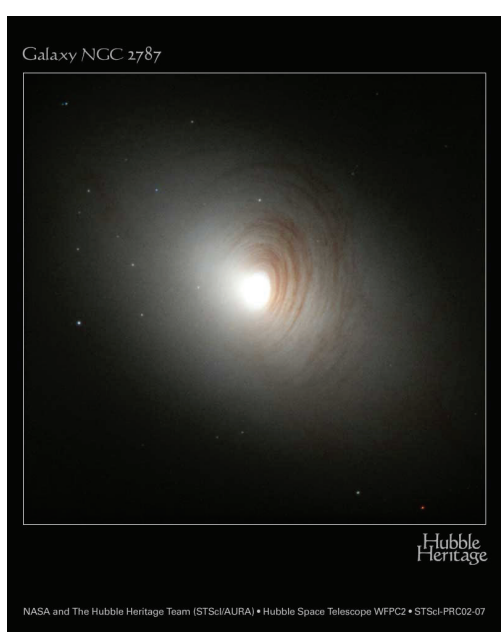

(b)

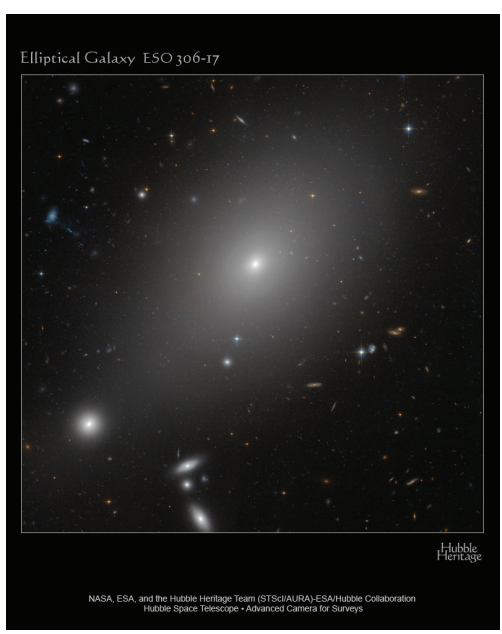

(d)

Figure 2: HST images of two S0 galaxies, NGC5866 (a) and NGC2787 (b), and two ellipticals, NGC1132 (c) and ESO306-17 (d). Credit: NASA, ESA, and The Hubble Heritage Team (STScI/AURA).

2.2. Automatic Galaxy Classifications. The main problem with visual galaxy classifications is that they are not reproducible. Although the classifications are done by specialists, visual galaxy classifications are not universal due to the lack of objectivity of the human eye. Automatic algorithms can provide reproducible galaxy classifications and give important clues to the origin and evolution of galaxies. Automatic classifications also have the advantage that biases can be fully understood and characterized based on extensive simulations of galaxies treated as real ones. Nevertheless, automated visual galaxy classifications can only provide broad galaxy classes. They are not as accurate as visual classifications made by trained observers.

Another problem with visual classifications is the time needed for detailed classifications of large galaxy samples. Present galaxy surveys such as Sloan Digital Sky Survey (SDSS; [25]) or Two Degree Field (2dF; [26]) have greatly increased the size of previous galaxy samples. Early galaxy catalogues contain several dozens, or even hundreds, of galaxies. In contrast, galaxy samples today can contain millions of galaxies. It is hard to give detailed morphological classifications of such large galaxy samples. Nevertheless, some contributions have been made in the last few years, and detailed visual morphological classifications of local galaxies are now available for small subsamples of the SDSS galaxy catalogue. Thus, Fukugita et al. [27] have visually classified a sample of 2253 galaxies, and Nair and Abraham [28] have also given detailed morphological types of 14034 nearby galaxies. Special mention should be made of the Galaxy Zoo project [29], which has provided visual classifications (not so detailed as the previously mentioned ones) for more than $4 \times 10^{7}$ galaxies by involving about $10^{5}$ classifiers $[29,30]$.

The amount of time needed for detailed visual classifications of galaxies makes the use of automated algorithms a must. Automatic classifications of galaxies can be divided into two broad groups: parametric and nonparametric. Parametric classifications reproduce galaxy measurements using parametric laws. One of the most extended parametric classifications classifies galaxies according to some properties of their structural parameters obtained through the fitting 


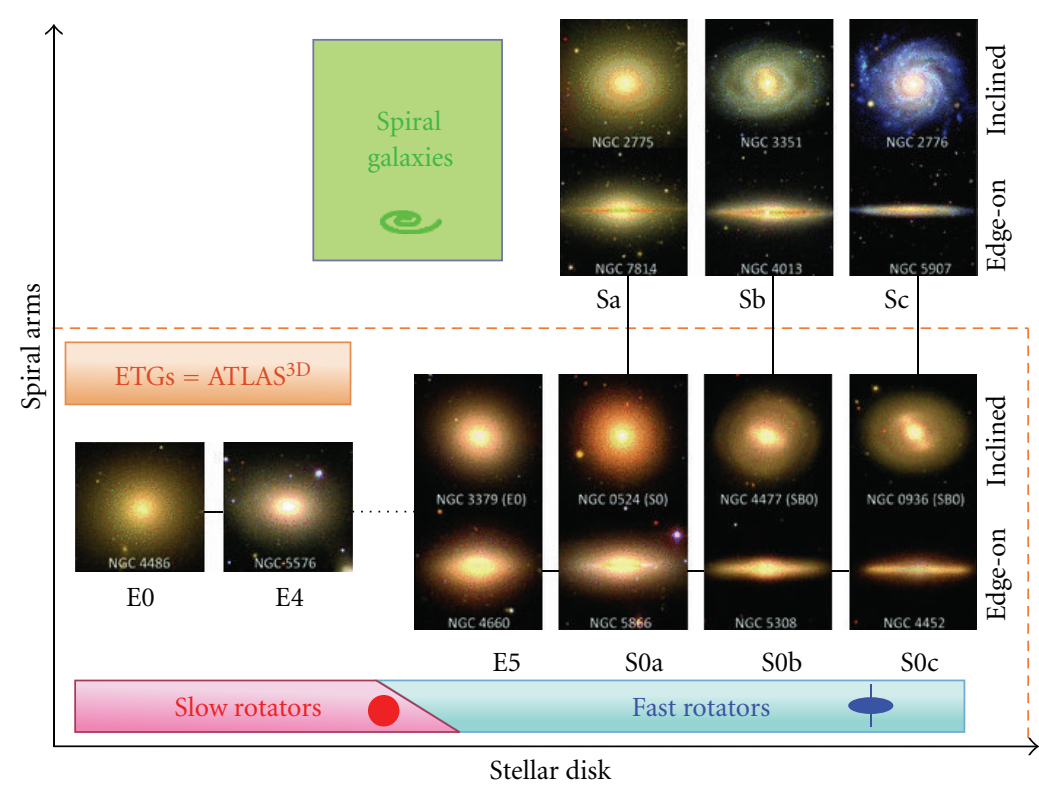

Figure 3: Galaxy classification scheme proposed by the ATLAS ${ }^{3 \mathrm{D}}$ project. Galaxies are classified as fast and slow rotators. Fast rotators form a parallel sequence to spiral galaxies. Credit: Cappellari et al. [18]. Reproduced by permission from WILEY.

of their surface brightness distribution. These algorithms assume that the luminosity distribution of a galaxy is the sum of the distributions of its individual components. The surface brightness of each component is modelled by a parametric law, which has to be strictly considered as an empirical fitting function (see the review of these algorithms in Section 3).

Nonparametric galaxy classifications are based on the measurements of a set of galaxy parameters that are correlated with the Hubble sequence. The advantage of this method is that it assumes no analytic models and can classify regular and irregular galaxies. Several galaxy parameters have been used for decades for galaxy classification. The colour of galaxies was one of the first parameters used in such classifications since colours are easy to obtain and correlate with galaxy morphological types. Thus, E and S0 galaxies show redder colours than spirals. This correlation is due to colours of galaxies reflecting the dominant constituent stellar population and this correlates with morphology. de Vaucouleurs [31] established the dependence of galaxy colour and morphological type. Colours have also recently been used for the morphological classification of large galaxy databases (e.g., [32] and references therein).

Spectra of galaxies also reflect their stellar populations and have been used for galaxy classification purposes. This property has been used in several spectroscopic galaxy classifications performed using certain features or full galaxy spectra in different wavelength and redshift ranges (e.g., [3343]). All these classifications show correlations between morphological and spectral classes. However, these correlations show wide scatter, particularly for E and S0 galaxies (see Figure 4 and [44] for the quantification of the scatter between morphological and spectral galaxy classes).

Galaxies can also be classified on the basis of their light distribution. Morgan $[45,46]$ used the central light concentration as a measure of the light distribution of galaxies.
Some years later, Fraser [47] defined light concentration indices as the ratio of the radii containing certain light percentages. These indices became popular and were used in several galaxy classifications (see [48-50]). Abraham et al. $[51,52]$ introduced the asymmetry of the galaxy light as another useful parameter in galaxy classification. They showed that different types of galaxies (early-type, latetype, and irregular) were located at different positions in the concentration-asymmetry (C-A) plane. Thus, early-type galaxies show a highly concentrated and symmetric light distribution. In contrast, the light distribution of irregular galaxies is less concentrated and homogeneous. A third parameter, smoothness (S), was proposed by Conselice et al. [53], given the CAS morphological classification system. Several other parameters such as the Gini coefficient (the Gini coefficient is a measures of the inequality of a distribution. This coefficient is a number between 0 for perfect equality and 1 for maximal inequality), galaxy ellipticity, or the M20 moment (the M20 moment is the second-order moment of the $20 \%$ brightest pixels of a galaxy.) have been included in nonparametric classification over the last decade [54-56]. All these parameters give some information about the galaxy shape.

It is very difficult to classify galaxies with classical approaches using more than three of the previous parameters. Several classification methods take into account the information contained in more than three parameters, but the final calibrations are done in three dimensions (e.g., [57]). This problem was recently solved by Huertas-Company et al. [58], who developed a generalization of the nonparametric classification that uses an unlimited number of dimensions. The method is based on a particular class of learning machines called support vector machines. The algorithm finds the optimal decision regions in a multidimensional space using a set of visually classified galaxies as a training 


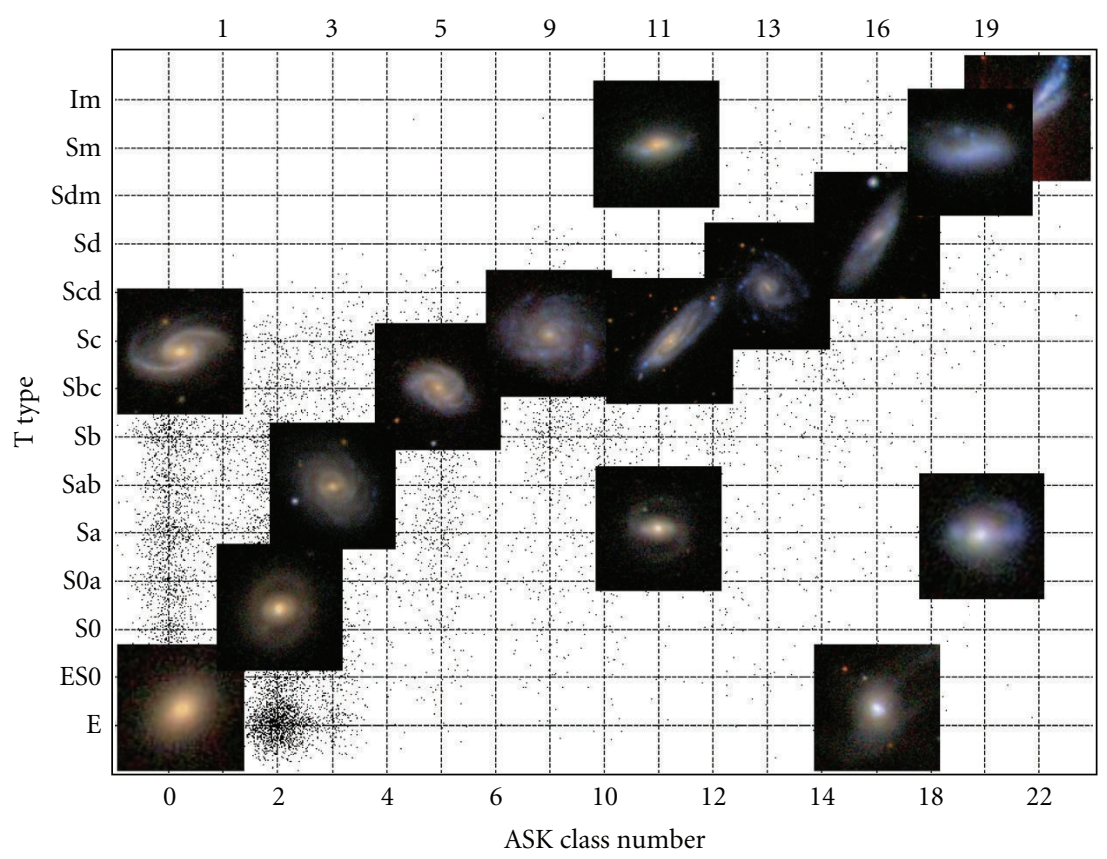

Figure 4: Scatter plot of Hubble versus spectral types. The sample of galaxies correspond to those galaxies classified visually by Nair and Abraham [28]. See more details in [44].

sample (see [59-61]). This classification algorithm has recently been applied to $\sim 700000$ galaxies from the SDSSDR7 spectroscopic galaxy catalogue [62]. All the galaxies were classified into four morphological classes: E, S0, Sab, and Scd. The novelty of this classification is that they assign to each galaxy a probability of being in the four morphological classes instead of assigning a single class. This Bayesian approach is better adapted to nature, where a continuous transition between different morphological types is observed. Good agreement was found between this automatic classification and visually based ones. Thus, good correlations were observed between the probability values given by this automatic classification and visual galaxy classes. For example, galaxies visually classified as ellipticals have on average a probability of $\sim 0.8$ of being $\mathrm{E}$ and $\sim 0.2$ of being S0. The other two probabilities of these galaxies turned to be almost zero (see [62]). This algorithm is one of the most appropriate for galaxy classifications of large galaxy samples due to the reasonable time required and the high level of agreement with other detailed visual classifications.

In the near future, all these new large galaxy samples will provide new insights in understanding the nature and evolution of lenticular and other morphological types of galaxies.

\section{Quantitative Morphology of Lenticular Galaxies}

From a structural point of view, galaxies can be simple systems formed by one main structural component, such as elliptical galaxies, or they can be more complex systems, such as spiral galaxies, showing structures such as spheroids, discs, bars, spiral arms, rings, and lenses. The luminosity distribution of a galaxy is the sum of the distributions of its individual components. The surface brightness distribution of the galaxies then provides information about their different structural components. It is assumed that each galaxy component can be modelled by a parametric law. Thus, the fit of the surface brightness distribution of galaxies by one or more components provides their structural parameters (size, scale, shape, and luminosity). Comparison of these structural parameters with different types of galaxies or galaxies of the same type but at different redshifts provides useful information for classifying galaxies and understanding their formation and evolution.

One of the basic problems of this quantitative morphology is prior knowledge, gained from solely photometrical information, of the number of structural components of the galaxies. This is crucial because the values of the resulting structural parameters of the galaxies depend on the number of structures used in the fit of their surface brightness distributions (e.g., [63]). This problem has special relevance in the case of S0 galaxies as they are objects between ellipticals and spirals. Usually, studies of large E and S0 datasets fit their surface brightness distributions by using only one component, obtaining global parameters such as scales, effective surface brightness, and the total luminosity of the galaxies. Nevertheless, S0 galaxies have at least two structural components (bulge and disc). Thus, their surface brightness should be fitted by using multicomponent models. The only way to solve this problem is through prior knowledge of the different structural components of the galaxies. This can be done by detailed studies of the photometric information of each galaxy before and/or after performing the decomposition of their surface brightness distribution. After the 
decompositions it should also be considered whether the parameters obtained are physically meaningful (see [64-66]).

Several multicomponent fitting algorithms have been developed for fitting the surface brightness distribution of galaxies (e.g., [64, 67-73]). Prieto et al. [64] developed one of these multicomponent techniques for fitting the surface brightness profiles of disc galaxies, including lenticulars. The process begins by analysing colour and colour-index images, which provides a qualitative idea of the numbers of components present in the galaxies. The scale lengths of these components were estimated taking into account the colourindex profiles of the galaxies. Confirmation of the assumed components was then obtained in the ellipticity and position angle radial profiles of the isophotes of the galaxies where the geometry of the different components, projected onto the sky plane, are well reflected. In this study and others using multicomponents fits, the surface brightness distribution of lenticular galaxies was fitted with a bulge, disc, bar, and lens. Below, I review the most popular mathematical laws used for fitting the surface brightness distribution of S0 galaxies. Figure 5 shows an example of 2D decompositions of the barred galaxy NGC 4643 using the multicomponent fitting method developed by [73].

3.1. Fitting Function of the Structural Components. Several mathematical functions have been used in order to fit the distribution of the stellar surface brightness of the different structural components of galaxies. These fitting functions include those for bulges, discs, lenses, and bars.

3.1.1. Bulge Fitting Functions. Historically, the first surface brightness models were proposed for fitting spheroidal galaxy components. The reason for this was that the first galaxies analysed were ellipticals. We can mention the surface-density models proposed by Reynolds [74], Hubble [75], King [76], Rood et al. [77], and Oemler [78]. For a good recent review of the mathematical laws proposed for fitting the surface brightness of galaxies see Graham [79].

One of the most popular fitting functions for describing the surface brightness of Es and bulges of S0s and spirals has been the de Vaucouleurs $r^{1 / 4}$ profile $[80,81]$. This model was proposed by de Vaucouleurs, who argued that it fitted the surface brightness of galaxies better than previous models for E galaxies. Indeed, since the work by de Vaucouleurs [82] for the bulge of M31, the $r^{1 / 4}$ law has been used for fitting the surface brightness of bulges of $\mathrm{S} 0$ and spiral galaxies. The radial variation of the surface intensity $\left(I_{d V}(r)\right)$ of this model is given by

$$
I_{d V}(r)=I_{d V, e} 10^{-7.669\left(\left(r / r_{e}\right)^{1 / 4}-1\right)},
$$

where $I_{d V, e}$ is the effective surface intensity of the galaxy and $r_{e}$ the effective radius, defined as the radius enclosing half of the total galaxy light. This model has been extensively used in surface brightness decompositions of ellipticals and bulges of S0 and spiral galaxies (e.g., [83-86]).

More recent studies have found deviations from the $r^{1 / 4}$ law of the surface brightness of the spheroidal components of individual galaxies (see [67, 87-89]), including the bulge of the Milky Way [90]. The universality of the $r^{1 / 4}$ profile for spheroidals began to be questioned after the work by Andredakis and Sanders [91], who found that bulges of latetype galaxies were better modelled by the Sérsic [92] law. This work was extended for a larger sample of galaxies of all morphological types, revealing that their bulges were accurately fitted by Sérsic profiles [93]. This was also confirmed by other researchers. Today, the Sérsic law is extensively used for modelling the surface brightness distribution of bulges in galaxies in the optical and near-infrared (e.g., [21, 63, 64, 94$105])$. The radial surface intensity $\left(I_{S}(r)\right)$ of the Sérsic law is given by

$$
I_{S}(r)=I_{S, e} e^{-b_{n}\left(\left(r / r_{e}\right)^{1 / n}-1\right)},
$$

where $I_{S, e}$ is the intensity at the effective radius $r_{e}$ that encloses half of the total light of the model. The parameter $n$ describes the shape of the profile, and the coefficient $b_{n}$ is given by $\approx 1.9992 n-0.327$ [106]. Notice that this profile is a generalization of the $r^{1 / 4}$ law. Thus, for $n=1$ the Sérsic profile is exponential, while $n=4$ is the de Vaucouleurs profile. Figure 6 shows the Sérsic radial profiles with different values of the $n$ parameter. For useful Sérsic reviews see Ciotti [107], Ciotti and Bertin [108], Simonneau and Prada [109], and Graham and Driver [110].

3.1.2. The Disc Component. Freeman [111] found that the surface brightness distribution of discs $\left(I_{D}(r)\right)$ in S0 and spiral galaxies could be modelled with an exponential function given by

$$
I_{D}(r)=I_{D, 0} e^{-r / h}
$$

where $I_{D, 0}$ is the central disc intensity and $h$ is the scale length of the profile. This exponential profile has been extensively used in surface brightness decompositions of S0 and spiral galaxies. Although some studies have used different fitting functions for the discs (e.g., [84]), they were not as popular as the exponential one.

Recently, detailed analyses of the external regions of disc galaxies have shown that not all galaxy discs show pure exponential surface brightness radial profiles. Most galaxies show truncations (downbending) or antitruncations (upbending) in their outermost regions. Thus, about $60 \%$ of galaxies have a break followed by downbending. Moreover, another $30 \%$ of galaxies have breaks followed by upbending. The shape of the surface brightness profiles of discs correlates with the Hubble type. Thus, downbending breaks are more frequent in late-type galaxies. In contrast, upbending breaks are more frequent in early-type galaxies (see [112]).

3.1.3. Bar Surface Brightness Laws. Bars are elliptical structures located in discs of galaxies. It is well known since pioneering morphological classifications of galaxies that strong bars are common in discs of S0 and spiral galaxies (see e.g., [12]). Early studies showed that the surface brightness profiles of bars in early-type galaxies are constant within the bar region while late-type galaxies show exponential-like bar profiles (see [113]). Nevertheless, it is still unclear which is 

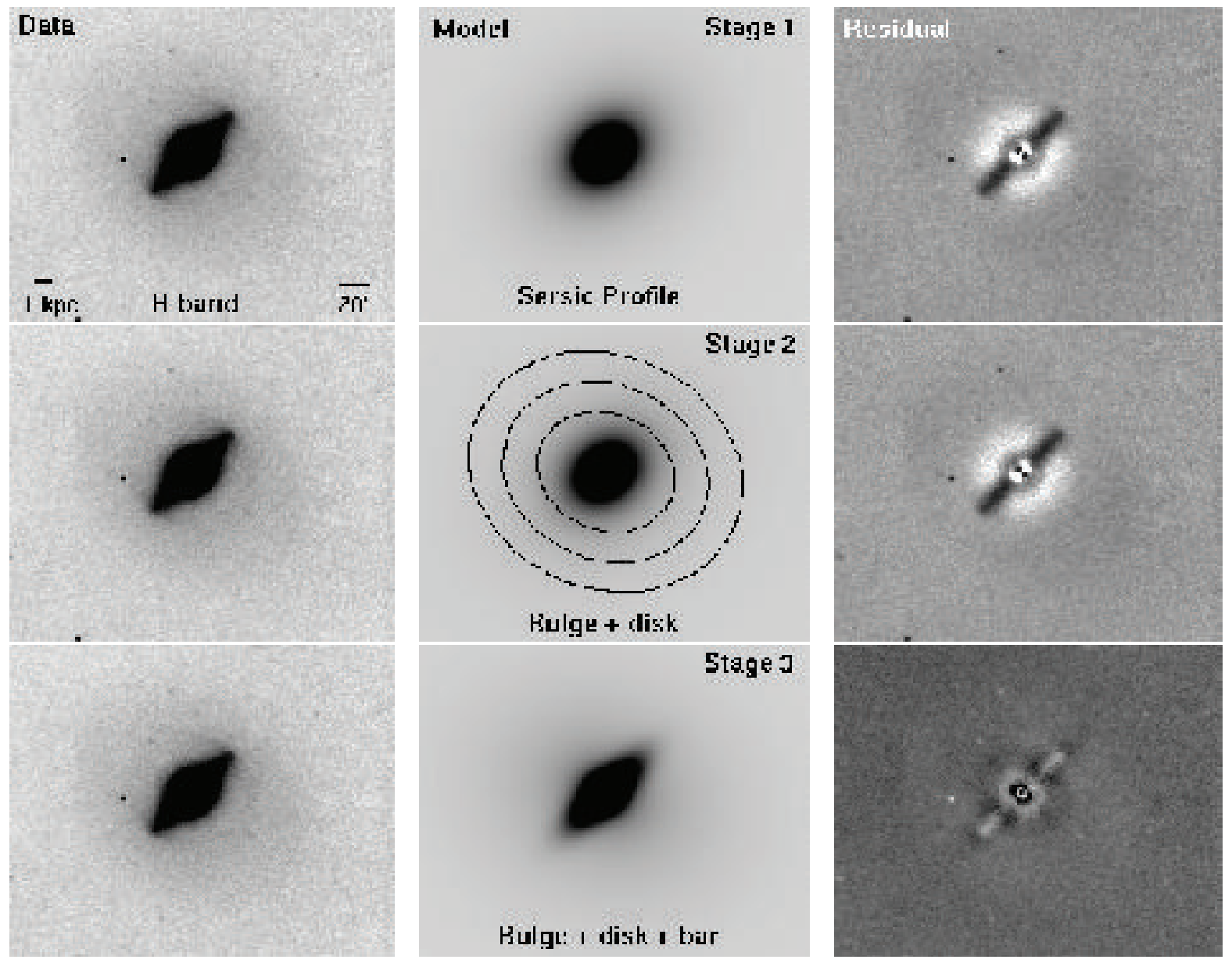

Figure 5: (left panels) r-band images the NGC4643. Sérsic (middle upper panel), Sérsic plus disc (middle middle panel), and Sérsic plus disc plus bar (middle-lower panel) models of the galaxy. (right panels) the corresponding residuals. Credit: Weinzirl et al. [73]. Reproduced by permission from the AAS.

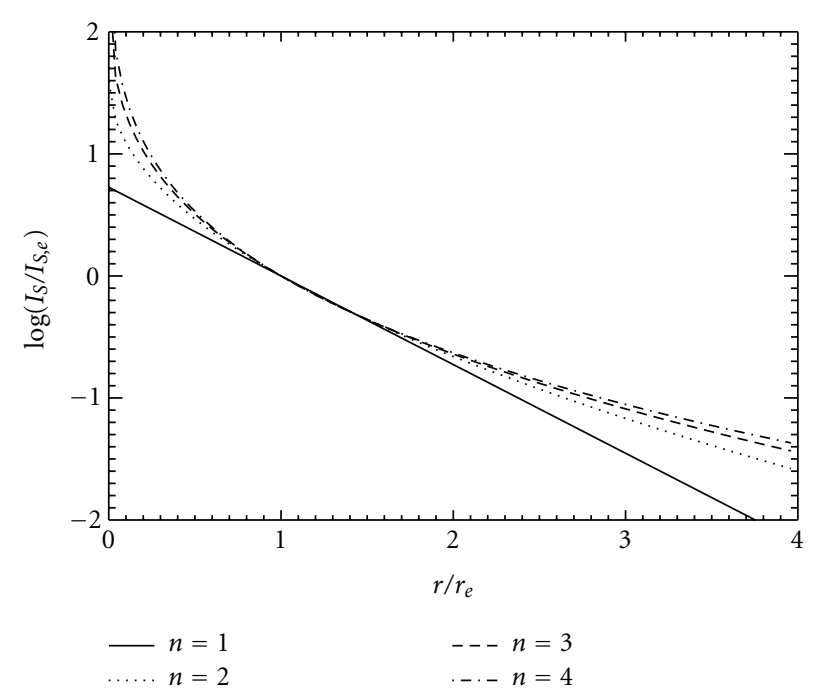

(a)

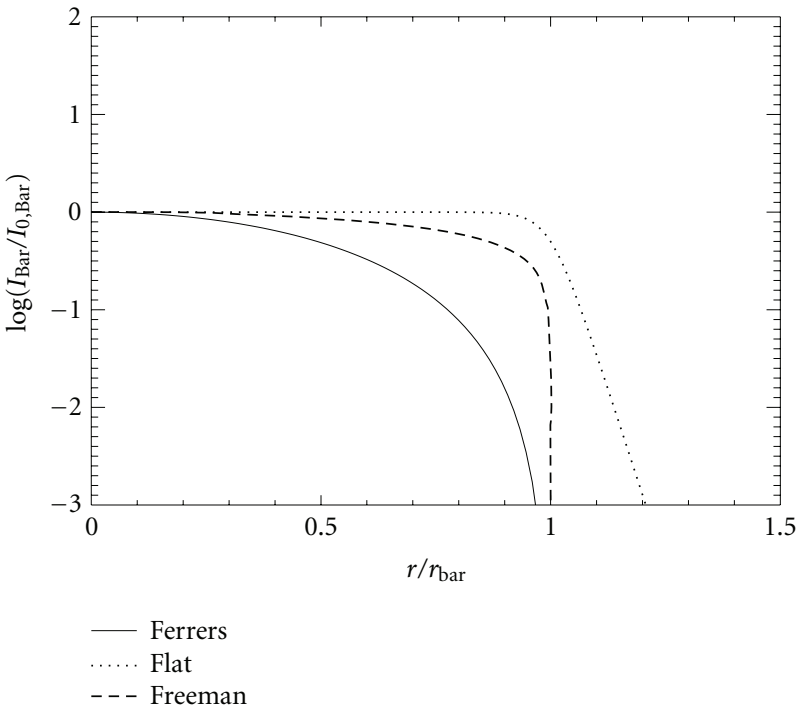

(b)

FIGURE 6: (a) Sérsic radial profiles with $n=1$ (full line), 2 (dotted line), 3 (dashed line), and 4 (dashed-dotted line). (b) Ferrers (full line), flat (dotted line), and Freeman (dashed line) radial profiles. 
the best mathematical fitting function describing the surface brightness radial profile of bars.

There are not many studies of the surface-brightness decomposition of barred galaxies. The large number of free parameters needed in these kind of fits makes them difficult. Fortunately, the number of studies including bars in the photometrical decomposition is increasing in the last years. Thus, some studies have used the Sérsic functions for fitting the observed surface brightness profile of bars (see [114]). Nevertheless, the most popular functions for fitting the observed surface brightness profiles of bars are Ferrers (see $[65,70]$ ), Freeman (see $[63,115]$ ), and flat (see $[63,64,115,116])$ profiles.

The radial surface intensity profile of a Ferrers ellipsoid $\left(I_{\text {Ferrers }}(r)\right)$ is given by

$$
I_{\text {Ferrers }}(r)=I_{F e, 0}\left(1-\left(\frac{r}{r_{\mathrm{bar}}}\right)^{2}\right)^{n_{\mathrm{bar}}+0.5},
$$

where $I_{F e, 0}, r_{\text {bar }}$, and $n_{\text {bar }}$ are the central surface intensity, length and a shape parameter of the bar, respectively. The total luminosity for a perfect ellipse is given by

$$
L_{\text {Ferrers }}=2 \pi I_{0} r_{\text {bar }}^{4} \int_{0}^{\infty} r\left(r_{\text {bar }}^{2}-r^{2}\right)^{n_{\text {bar }}+0.5} d r .
$$

In the case of $n_{\text {bar }}=2$, the previous integral has a primitive, and the total luminosity $\left(L_{\text {Ferrers }}\right)$ is given by

$$
L_{\mathrm{Ferrers}}=\pi I_{F e, 0}\left(1-\epsilon_{\mathrm{bar}}\right) r_{\mathrm{bar}}^{2} \frac{\Gamma(7 / 2)}{\Gamma(9 / 2)} .
$$

The radial surface intensity profile $\left(I_{\text {Freeman }}(r)\right)$ of a Freeman bar is given by

$$
I_{\text {Freeman }}(r)=I_{F r, 0} \sqrt{1-\left(\frac{r}{r_{\mathrm{bar}}}\right)^{2}} .
$$

$I_{F r, 0}$ and $r_{\text {bar }}$ are the central surface intensity and length of the bar, respectively (see [117]). The Freeman bar is a particular case of the Ferrers bar with $n_{\mathrm{bar}}=0$. In this case, the total luminosity for a Freeman bar is given by

$$
L_{\text {Freeman }}=\frac{2}{3} \pi\left(1-\epsilon_{\text {bar }}\right) I_{F r, 0} r_{\text {bar }}^{2} .
$$

The radial surface intensity profile of a flat bar $\left(I_{\text {Flat }}(r)\right)$ is

$$
I_{\text {Flat }}(r)=I_{F l, 0}\left(\frac{1}{1+e^{\left(r-r_{\text {aar }}\right) / r_{s}}}\right),
$$

where $I_{F l, 0}$ and $r_{\mathrm{bar}}$ are the central surface intensity and the length of the bar. For radii greater than $r_{\mathrm{bar}}$ the profile falls with a scale length $r_{s}$ [116]. In this case, the total luminosity of this bar is given by

$$
L_{\text {Flat }}=-2 \pi I_{F l, 0}\left(1-\epsilon_{\mathrm{bar}}\right) r_{s}^{2} L i_{2}\left(-e^{r_{\mathrm{bar}} / r_{s}}\right),
$$

where $L i_{2}$ is the dilogarithm function (also called the Jonquiere function).

The surface radial profiles of Ferrers, Freeman and flat bars with the same bar radius can be seen in Figure 6. It is not clear which of the previous functions better fit the observed surface brightness distribution of bars. Probably, the type of bar is correlated with the morphological type of the galaxy (see [118]). Nevertheless, more investigation is needed in this subject.

3.1.4. Lens Component. Lenses are photometric components commonly observed in lenticular and some early-type galaxies. In contrast, few late-type galaxies show lenses. Kormendy [119] found that $54 \%$ of SB0-SBa galaxies have lenses. A similar fraction of lenses has been observed in nonbarred S0 galaxies $[65,66,70]$. Their surface brightness radial intensity $\left(I_{l}(r)\right)$ is characterized by smooth luminosity gradients with a very sharp cut-off. Duval and Athanassoula [120] found a lens in the luminosity profile of the galaxy NGC 5383. This lens was fitted by the expression

$$
I_{l}(r)=I_{l, 0}\left(1-\left(\frac{r}{r_{l}}\right)^{2}\right), \quad \text { for } r<r_{l},
$$

where $I_{l, 0}$ is the surface intensity of the lens at the centre and $r_{l}$ is the size of the lens. This expression was also used by Prieto et al. [64] and Aguerri et al. [63] for fitting the surface brightness radial profiles for some lenticular galaxies. In multicomponent fits, lenses represent a small percent (less than 10\%) of the total light of the galaxies (see $[63,64])$. The origin and evolution of lenses are unclear. Nevertheless, Kormendy [119] has argued that lenses in lenticulars originate as bars. He suggested that bars evolve into lenses through some internal process.

\section{Properties of the Structural Components of Lenticular Galaxies}

The relations between the structural parameters have been extensively studied in the literature in order to understand the formation and evolution processes of different galaxy components (for a recent review see [79]). I review below some of the structural relations given in the literature for samples of galaxies including S0s. In this paper, I took into account galaxy samples with multicomponent surface brightness decompositions. Thus, the structural parameters of all galaxies have been obtained in a homogeneous way.

\subsection{Bulge Structural Parameters}

4.1.1. Correlations with Hubble Type. Andredakis et al. [93], using an extension of the model-independent decomposition method introduced by Kent [121], found correlations of certain structural parameters of bulges with galaxy Hubble type. In particular, using near-infrared images, they found that the bulges of early-type spiral galaxies had a Sérsic shape parameter of $n \approx 4$, while bulges from late-type galaxies showed $n \approx 1$. This continuous relation indicated a wide diversity concerning the shape parameter in galaxy bulges, similar to ellipticals (e.g., [122-124]). This diversity also indicated that the different types of bulges were not well fitted using a single law (such as the de Vaucouleurs law). This 
trend was later confirmed by other works (e.g., [94, 97, 125129]).

Recent surface brightness decomposition of large galaxy samples, including S0s, show that the correlation between $n$ and Hubble type is not so evident. For example, MendezAbreu et al. [103] found no correlations between the bulge parameters $\left(r_{e}, n\right.$, and $\left.\mu_{e}\right)$ and Hubble type for a sample of 148 unbarred S0-Sb bulges (see Figure 7). Laurikainen et al. [21] found that $n$ was almost constant from $\mathrm{SO}^{-}$to Sa bulges of galaxies and was only slightly larger for S0-Sa galaxies than for late-type bulges. Similar results have been found in other samples with few S0 galaxies (see $[63,130]$ ). All these studies indicate that there is a wide scatter of $n$ values for $\mathrm{S} 0$ galaxies. Thus, some bulges of S0 galaxies show $n$ values similar to those of Es $(n \approx 4)$. But there is also a significant fraction of bulges of S0 galaxies showing similar values of the shape parameter $(n<2)$ to those of late-type (see, e.g., $[63,65,73,131]$ and Figure 7$)$.

4.1.2. The Fundamental Planes and Their Projections. Dynamically hot systems are not scattered randomly in the parameter space defined by their effective surface brightness $\left(\mu_{e}\right)$, half-light radius $\left(r_{e}\right)$, and central velocity dispersion $\left(\sigma_{0}\right)$. On the contrary, they follow a tight correlation called the fundamental plane (FP; $[132,133])$. The bulges of S0 galaxies are located in the same FPs as E galaxies [63, 103] or with a slight offset [134]. No statistically significant difference has been observed between the FP defined by bulges of S0s or early-type spirals [103]. These studies have also shown that the FP of bulges of S0 galaxies are independent of their $n$ Sérsic values. Thus, So bulges with $n \approx 1$ and $n \approx 4$ are located in the same FP (see [63]).

Kormendy [84] found a strong correlation between the surface central brightness of $\mathrm{E}$ galaxies and their effective radius. This relation is also kept when the mean effective surface brightness $\left(\left\langle\mu_{e}\right\rangle\right)$ is used. This is the so-called Kormendy relation (KR) and is one of the projections of the FP. Several authors have shown that bulges of S0 galaxies follow a similar KR as E galaxies. In contrast, bulges of latetype galaxies show a large offset with respect to the KR of Es $[85,88,93,101,135]$. For a large sample of S0 galaxies, Laurikainen et al. [21] found that the slopes of the KR of bulges of S0 and E galaxies were similar. But, the zero points of the relations were not the same. They reported a small offset of about $0.3 \mathrm{mag}$. This offset was not observed when bulges brighter than $M_{K}=-22.7$ were considered (see Figure 8). Similarly, Barway et al. [136] also found that bright S0 galaxies $\left(M_{K}<-24.5\right)$ followed the same KR as Es in the Coma cluster, while the KR of faint S0s showed an offset with respect to E galaxies.

Another projection of the FP is the so-called FaberJackson (FJ) relation ([see 144]), which relates the luminosity $(L)$ of spheroidal systems to their central velocity dispersion $\left(\sigma_{0}\right)$. Several studies have found that this relation depends strongly on the luminosity of the galaxies (see [137] and references therein). Thus, Matković and Guzmán [138] found that bright elliptical galaxies show a tight FJ relation given by $L \propto \sigma_{0}^{4}$, while faint early-type galaxies follow a significantly shallower relation $\left(L \propto \sigma_{0}^{2.01}\right)$. Bulges of S0 and Sa galaxies follow a similar FJ relation to that of bright Es $\left(L \propto \sigma_{0}^{4.2} ;[103]\right)$. The deviation from the elliptical FJ relation is due to bulges fainter than $M_{B}=-20$ (see $[103,130]$ ).

4.1.3. Other Relations between Bulge Effective Radius, Luminosity, and Sérsic Shape Parameter. The Sérsic shape index $n$ has a correlation with the total luminosity of bulges $\left(M_{\text {bulge }}\right)$ for spiral galaxies (see $[21,96,97,123,139]$ ). Thus, brighter bulges show larger values of $n$. This correlation is also shown for E galaxies (e.g., [124]). In contrast, bulges of S0 galaxies do not show a statistically significant relation between $n$ and $M_{\text {bulge }}$ (see [21]).

The effective radius $\left(r_{e}\right)$ of bulges of early-type spirals and S0 correlates with the bulge luminosity (see [65, 130]). Nevertheless, bulges of spirals and lenticulars show a different $r_{e}-M_{\text {bulge }}$ relation from that for elliptical galaxies (see [140]). In particular, for a given bulge luminosity, bulges of S0 galaxies are more compact than those Es with the same luminosity. This difference was argued by Laurikainen et al. [21], who concluded that bulges of S0s galaxies were photometrically different from those of Es (see Figure 9).

Classical bulges can be distinguished from pseudobulges (Many bulges show disk-like features that do not resemble hot stellar systems. These kind of bulges are usually called pseudobulges (see [141]).) by their scaling relations (e.g., $[141,142])$. In a series of papers, Fisher and Drory $[131,143]$ have shown that classical bulges (defined as those with $n>2$ ) follow the same relations as E galaxies between the effective radius, luminosity and Sérsic shape parameter. In contrast, these relations are not followed for pseudobulges (those with $n<2$ ). They conclude that the Sérsic shape index can be used in order to distinguish between classical bulges and pseudobulges (see [141] for a definition of pseudobulge). This classification is independent of the Hubble type. Thus, their galaxy sample contains lenticular bulges, most of them being of the classical type. But a few of their lenticular bulges are catalogued as pseudobulges. This indicates the wide variety of bulges of lenticular galaxies.

4.1.4. The Intrinsic Shape of Bulges. In comparison with the large number of works investigating the correlations between the bulge parameters of $\mathrm{S} 0$ and spiral galaxies, little attention has been given to the three-dimensional shape of bulges (e.g., [144-148]). Independently of their internal structure, the bulges of lenticulars and spiral galaxies have been traditionally considered as ellipsoidal stellar systems located at the centre of galaxies. Bulges and discs are concentric with the galactic centre and share the same polar axis. In this framework, the twisting of bulge isophotes [144, 149] and misalignment between the major axes of the bulge and disk $[145,147]$ are not possible if the bulge and disk are both oblate. They were interpreted as a signature of bulge triaxiality. This idea has been supported by the presence of noncircular gas motions (e.g., [150-153]) and a velocity gradient along the galaxy minor axis [154-156].

In a series of papers, Méndez-Abreu et al. [103, 104] have studied the 3D shape of the bulges of a large sample of S0 and 


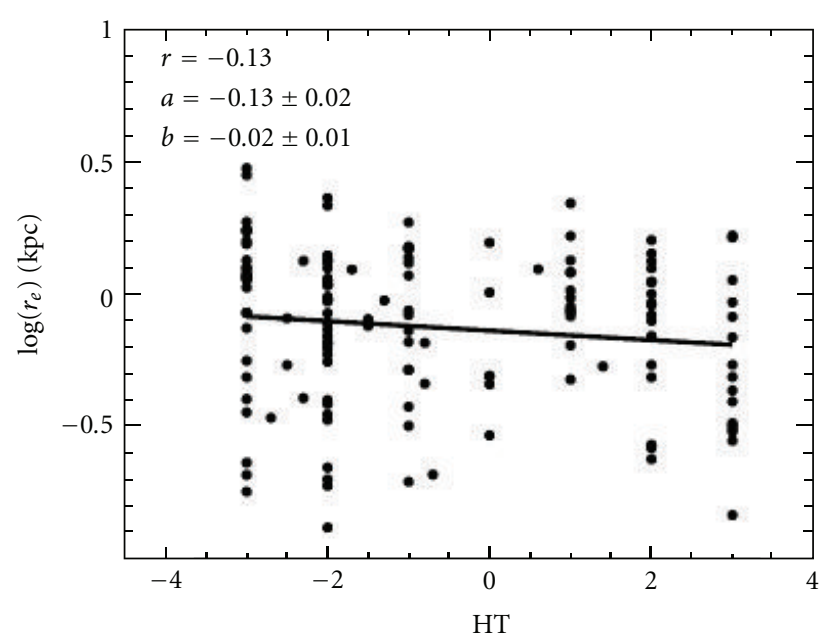

(a)

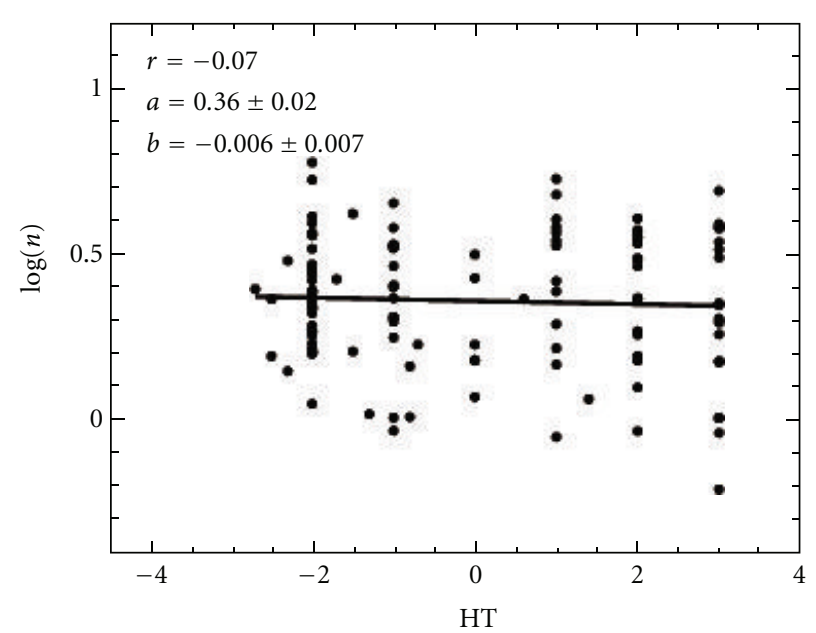

(c)

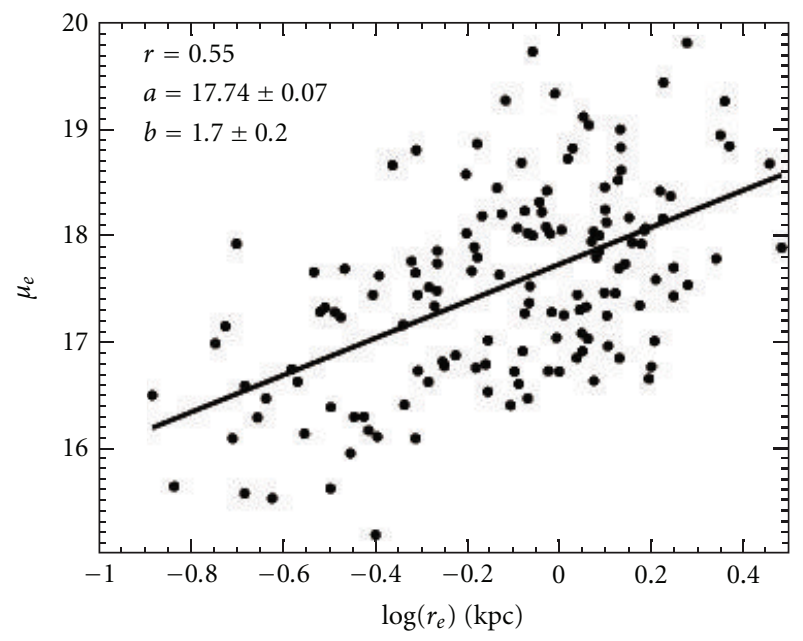

(e)

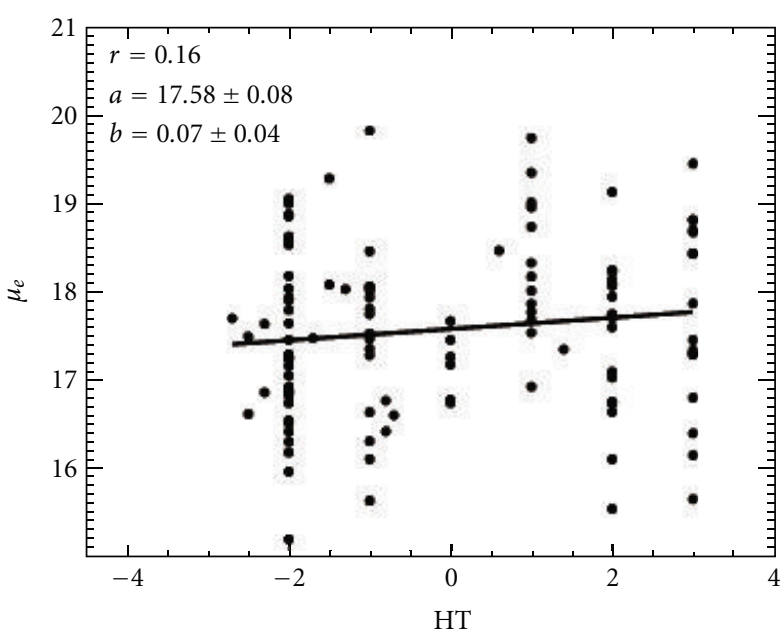

(b)

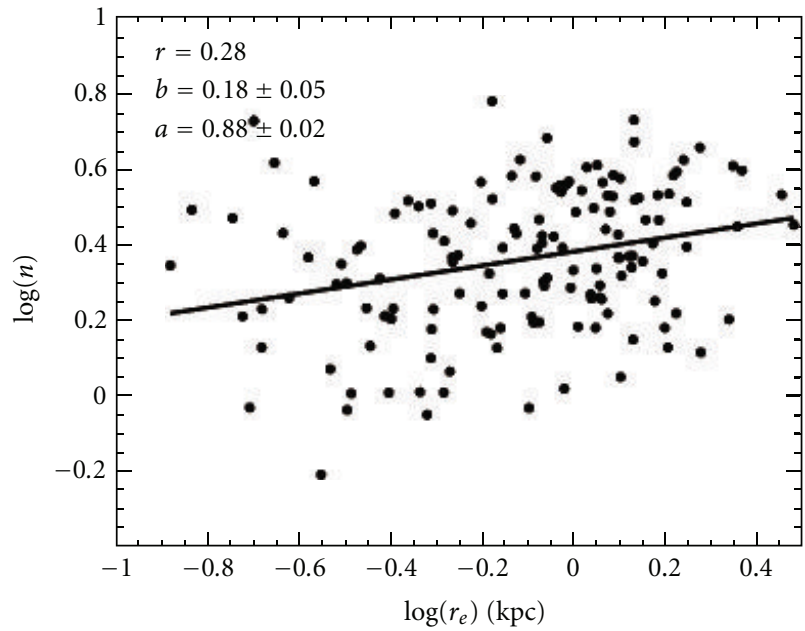

(d)

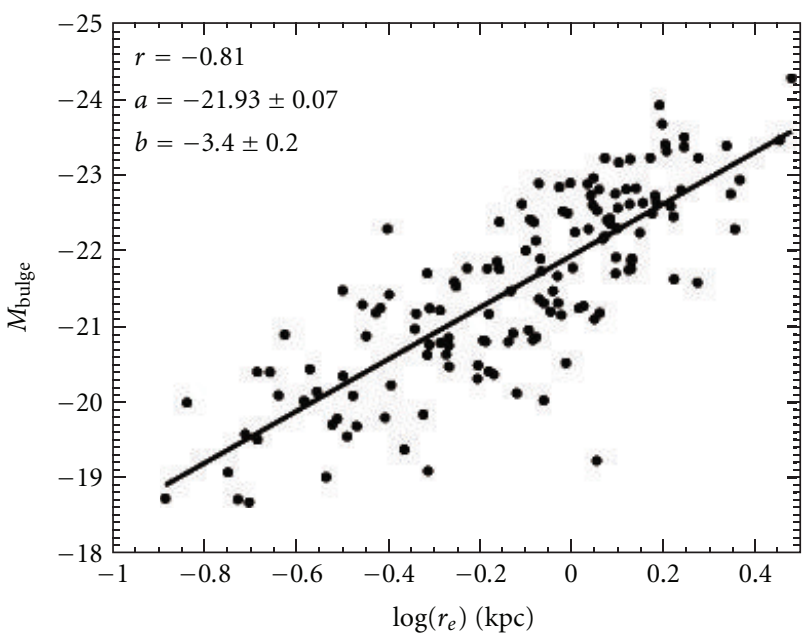

(f)

FIGURE 7: Correlations between the bulge parameters for a sample of lenticulars and early-type spirals. Correlation between the Hubble type and effective radius (a), effective surface brightness (b), and Sérsic parameter (c). Correlations between the effective radius of the bulge and the Sérsic parameter (d), effective surface brightness (e), and absolute magnitude (f). The full line represents the best linear fit. The Pearson correlation coefficient $(r)$ is also indicated. See [103] for more details. 


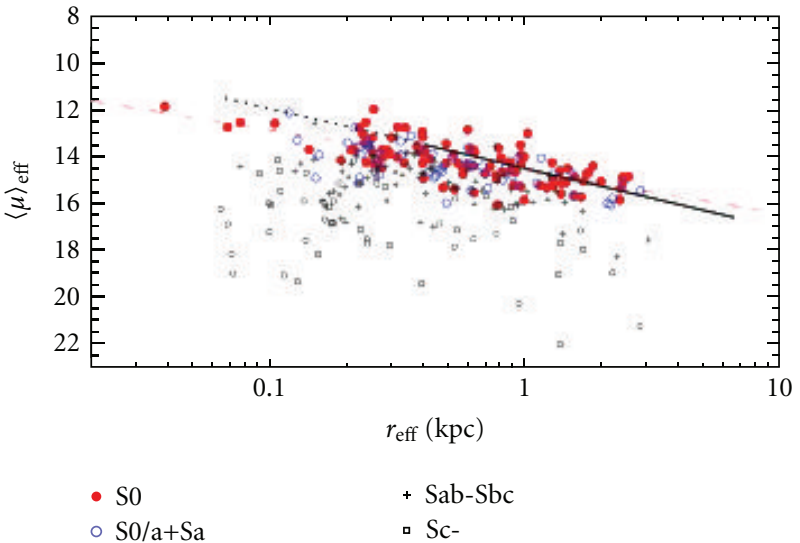

(a)

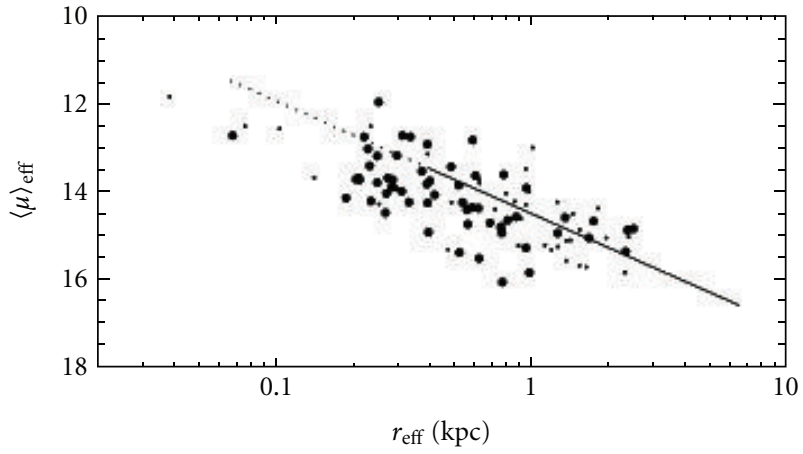

- S0: bar

- S0: non bar

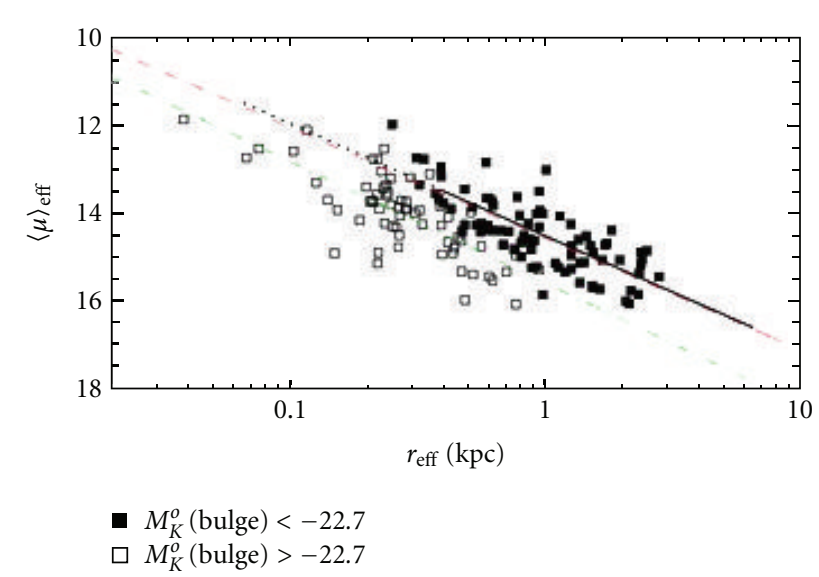

(b)

(c)

FIGURE 8: The Kormendy relation for bulges of S0, early-type and late-type spirals. In all panels the full line represents the KR of elliptical galaxies in the Coma cluster. The dotted black line corresponds to the extension of the KR of ellipticals towards galaxies with smaller effective radius. The red dashed line of the upper panel represents the KR for the S0 bulges. The red and green dashed lines in the lower panel show the KR for S0 bulges brighter and fainter than $M_{k}=-22.7$, respectively. Credit: Laurikainen et al. [21]. Reproduced by permision from WILEY.

early-type spiral galaxies. They found that $80 \%$ of bulges in unbarred lenticulars and early-type galaxies were not oblate but triaxial ellipsoids. Their mean axial ratio in the equatorial plane was $\langle B / A\rangle=0.85$. This was in agreement with early findings by Bertola et al. [145] and Fathi and Peletier [157]. In addition, Méndez-Abreu et al. [103] found no significant dependence on the probability distribution function of $B / A$ and other galaxy properties, such as morphological type, light concentration, or luminosity of bulges.

Méndez-Abreu et al. [104] have found that most of the bulges of S0 and early-type spirals are flattened along their polar axis. Only $18 \%$ of the bulges had a probability larger than $50 \%$ of being elongated along their polar axis. In this study, the triaxiality parameter $\left(T=\left(1-(B / A)^{2}\right) /(1-\right.$ $\left.(C / A)^{2}\right)$, where $A, B$, and $C$ were the lengths of the longest, intermediate and shortest semiaxes of the bulges; see [158]), was also analysed with the result that the triaxiality distribution of the bulges was strongly bimodal. This bimodality was driven by the Sérsic shape parameter and the $B / T$ ratio of the bulges. Thus, bulges with $n<2$ and $B / T<0.3$ exhibit a larger fraction of oblate axisymmetric bulges, a smaller fraction of triaxial bulges, and fewer prolate axisymmetric bulges with respect to bulges with $n>2$ and $B / T>0.3$ (see [104] and Figure 10). No correlation was found between the intrinsic shape of bulges and other galaxy properties, such as the total luminosity or central velocity dispersion.

\subsection{Disk Structural Parameters}

4.2.1. Correlations with Hubble Type. Several works found no correlation between the disc scale length and the Hubble type over a wide range of morphological types, including S0 discs (see [94, 97, 98, 103, 111, 129, 130]). Thus, the scale length of the discs was independent of the Hubble type of the galaxy. Freeman [111] discovered that the central surface brightness of the discs in spiral and S0 galaxies has a constant value of $\left(\mu_{0}=21.65 \mathrm{mag} / \mathrm{arcsec}^{2}\right)$ with only $0.3 \mathrm{mag} / \operatorname{arcsec}^{2}$ of standard deviation. Nevertheless, this was not confirmed in later studies. Thus, late-type spirals show fainter central surface brightness than early-type ones (see 


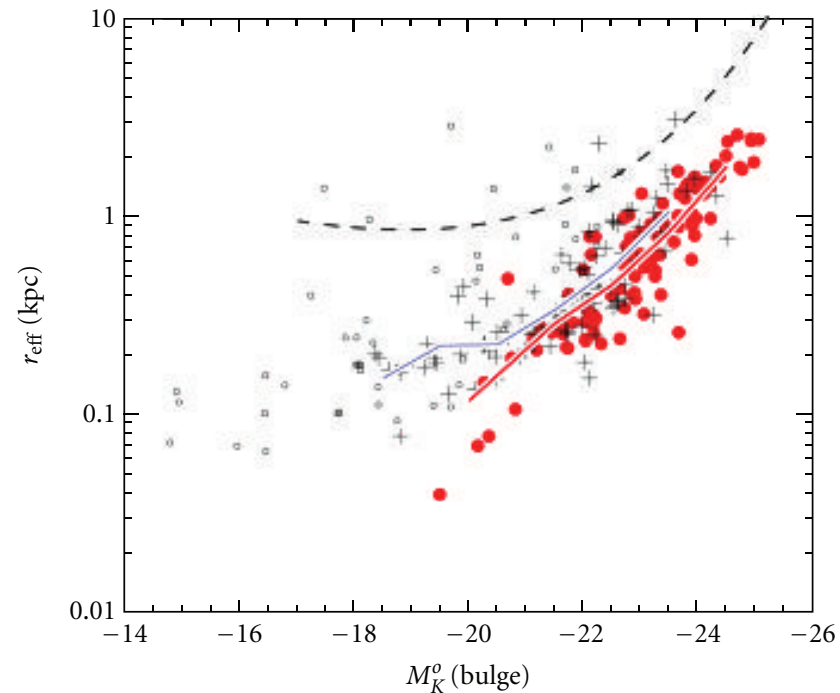

- S0

+ Sa-Sbc

- Sc-

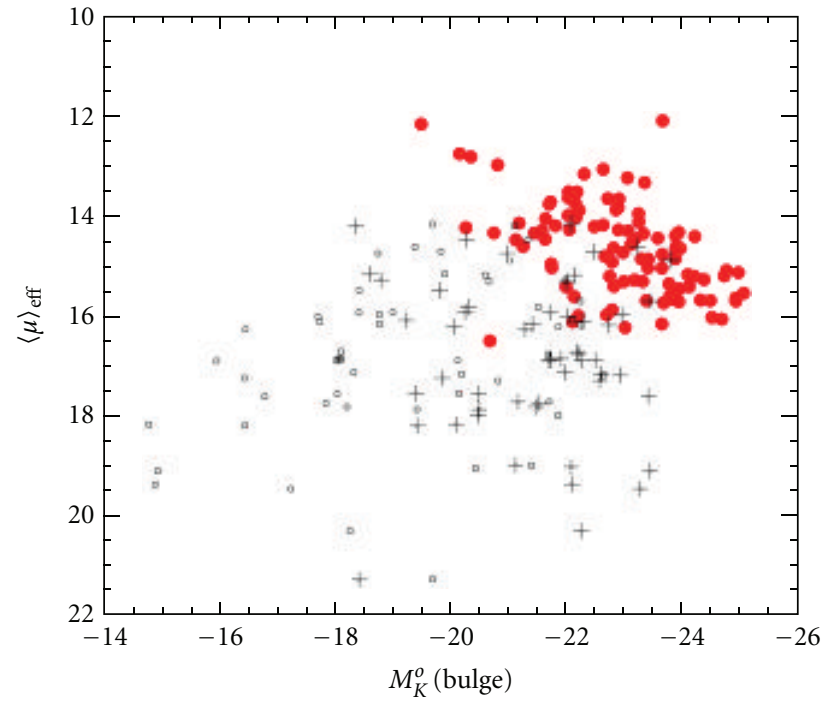

- S0

+ Sa-Sbc

- Sc-

(a)

(b)

FIGURE 9: Effective radius (a) and effective surface brightness (b) of bulges of S0 (red points), early-type (crosses), and late-type (squares) spirals as a function of the bulge absolute magnitude. The dashed line in the left panel corresponds to the relation followed by ellipticals galaxies from the Coma cluster. The continuous lines in the left panel correspond to the mean values of the data points in one- magnitude bins: S0s (red line) and Sa-Sbc (blue line). Credit: Laurikainen et al. [21]. Reproduced by permision from WILEY.

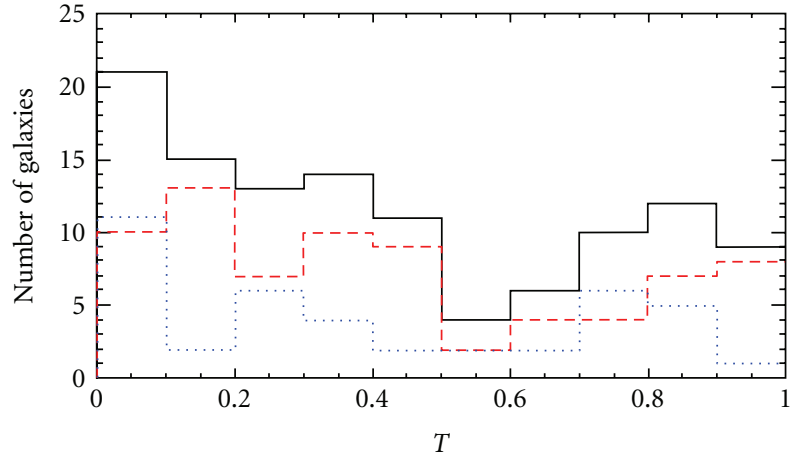

(a)

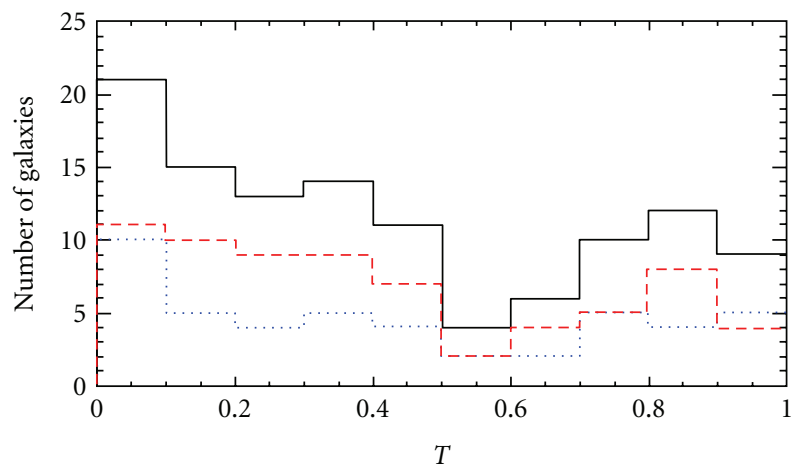

(b)

Figure 10: Distribution of the triaxiality parameter $T$ for the 115 bulges of $S 0$ and early-type galaxies. Values of $T=0$ indicate oblate bulges and $T=1$ prolate ones. The distributions of $T$ for bulges with $n<2$ (dotted line) and $n>2$ (dashed line) are shown in (a). (b) shows the $T$ distributions for bulges of galaxies with $B / T<0.3$ (dotted line) and $B / T>0.3$ (dashed line). See [104] for more details.

$[94,96,129,130])$. The correlations of the disc structural parameters for a large sample of bulges from S0 and earlyype galaxies are shown in Figure 11.

4.2.2. Correlations between $h, \mu_{0}$, and Disc Luminosity. The scale length of discs of spiral and lenticular galaxies correlates with the disc luminosity. Thus, larger discs are located in brighter ones (see $[21,103,130])$. The discs of S0 and spiral galaxies also show a correlation between $\mu_{0}$ and $h$ in the sense that larger discs have a fainter central surface brightness $[21,63,103,130]$. In both relations the discs of spiral galaxies and lenticulars are located in the same regions, indicating that they are similar (see [21] and Figure 11).
4.2.3. The Tully-Fisher Relation. The Tully-Fisher relation (TFR; [159]) is a correlation between the asymptotic circular velocity $\left(V_{c}\right)$ of disc galaxies and their integrated stellar luminosity $(L)$. It is well known that the scatter and the zero point of this relation depend on the Hubble type. Thus, late-type spirals show a tight TFR with low dispersion $(\sigma \approx$ $0.3)$. In contrast, $\mathrm{Sa}$ and $\mathrm{Sb}$ galaxies show TFRs with wider scatter and different zero points from those of Sc ones (e.g., $[160,161])$.

The TFR of S0 galaxies has been less investigated in the literature owing to the difficulty in obtaining the asymptotic circular velocity of these gas-poor galaxies. Nevertheless, several studies indicate that the TFR of S0 galaxies shows a 


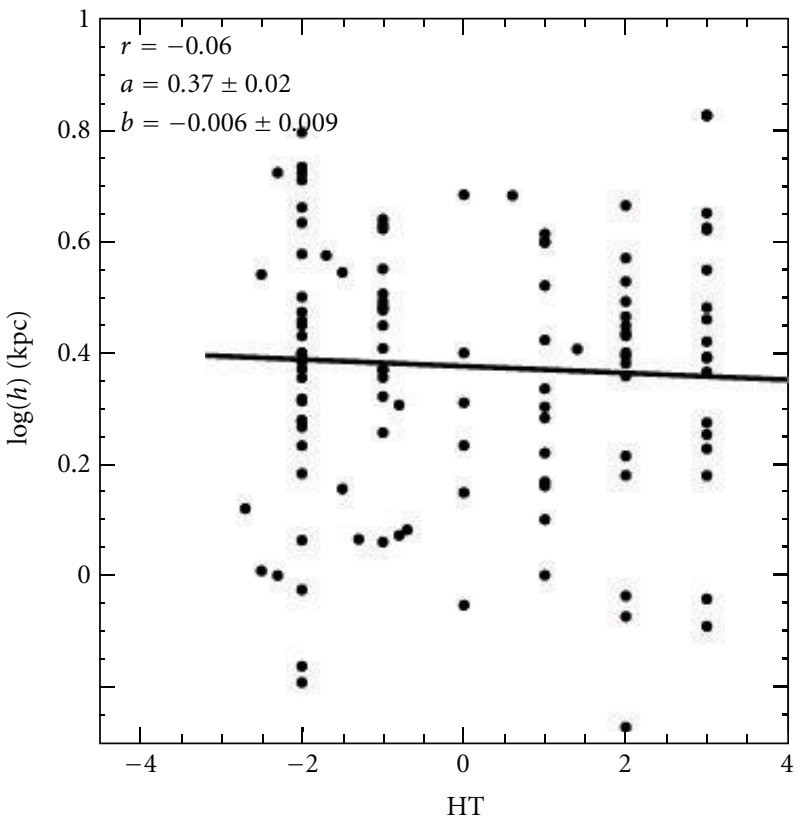

(a)

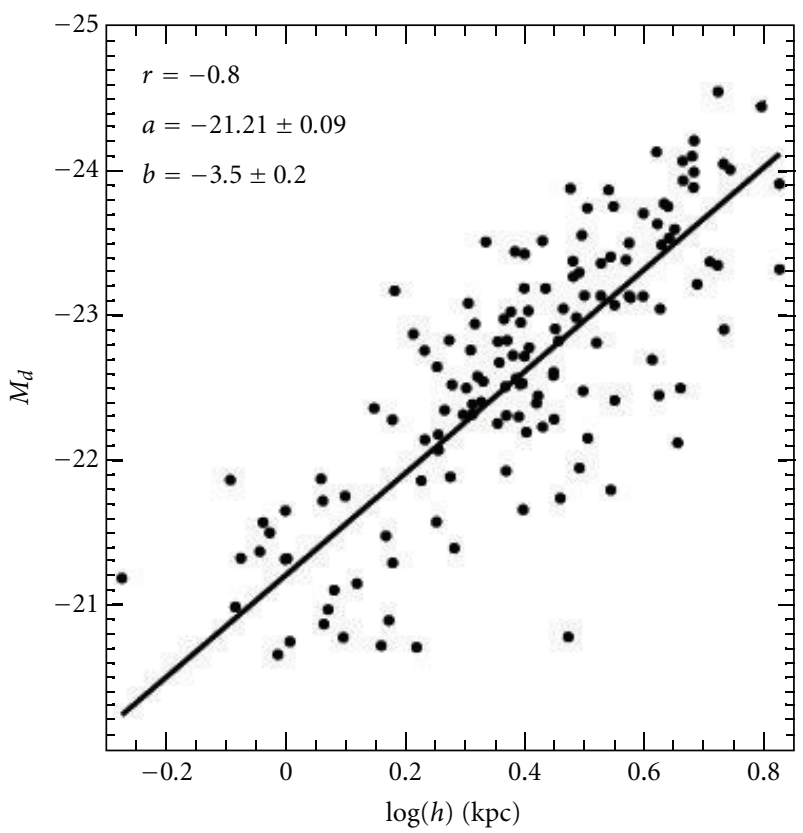

(c)

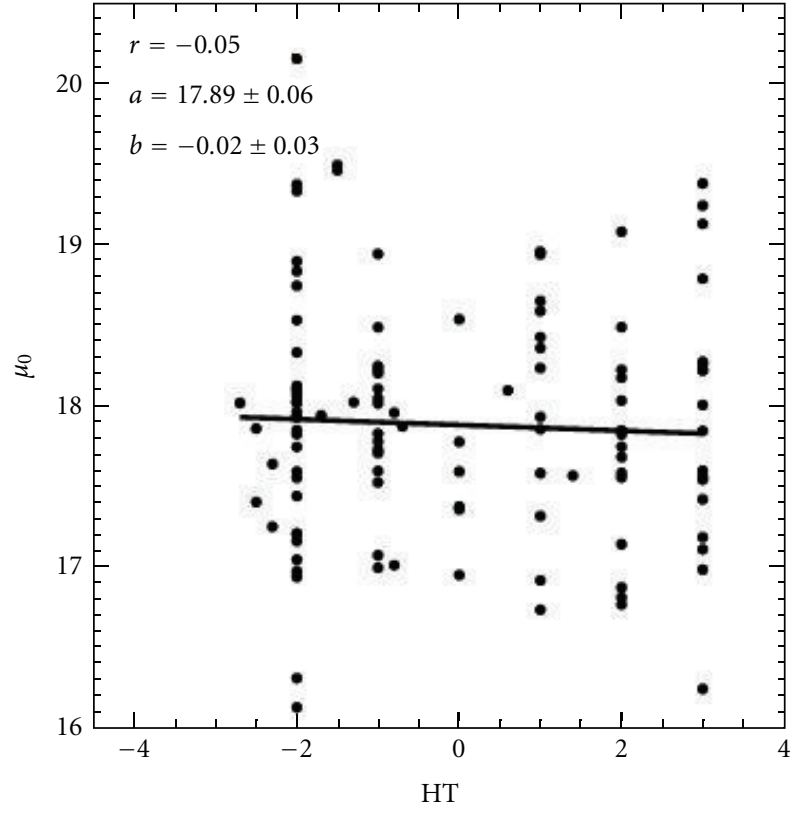

(b)

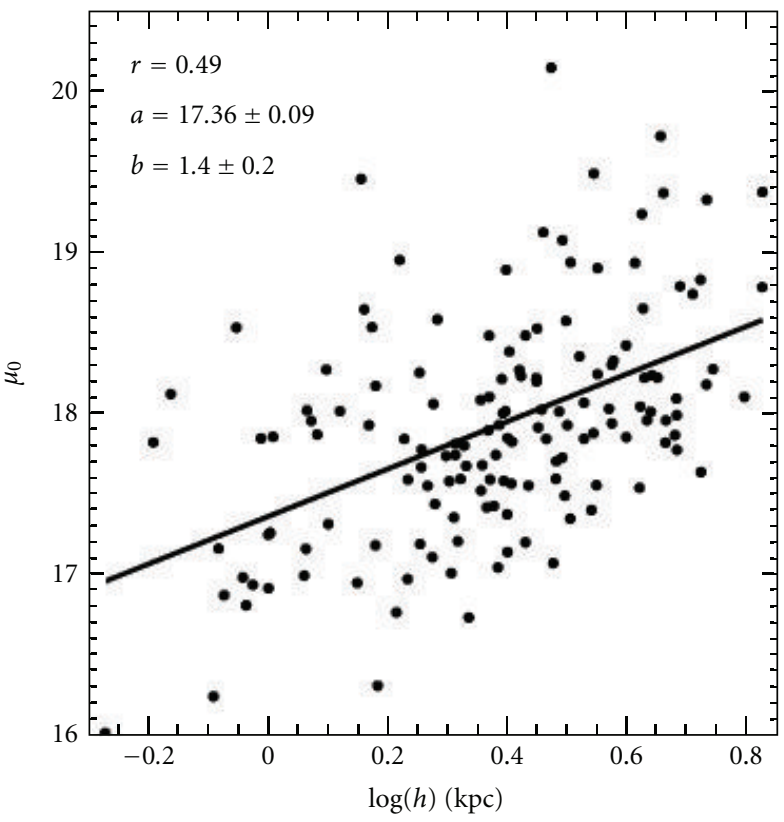

(d)

Figure 11: Correlations between the disc parameters for a sample of lenticulars and early-type spirals. Correlation between the Hubble type and disc scale length (a) and the central surface brightness (b). Correlations between the disc scale length and the absolute magnitude of the disc (c) and the central surface brightness (d). The full line represents the best linear fit $(y=a+b x)$. The Pearson correlation coefficient $(r)$ is also indicated. See [103] for more details.

similar slope to that of late-type ones but with a different zero point (see Figure 12). This offset indicates that, for a given circular velocity, S0 galaxies are fainter than late-type ones (see [162-167]). The offset is the expected one if the progenitors of present $\mathrm{S} 0$ galaxies were late-type galaxies that have stopped their star formation a few Gyr ago.
4.2.4. The Rotational Velocity-Size Plane. The distribution of disc galaxies in the rotational velocity-size $\left(V_{c}-R\right)$ plane provides information about galaxy formation. These two parameters are related with the total angular momentum $(J)$ and mass $(M)$ of galaxies, which have been repeatedly invoked as the fundamental parameters in galaxy formation. 


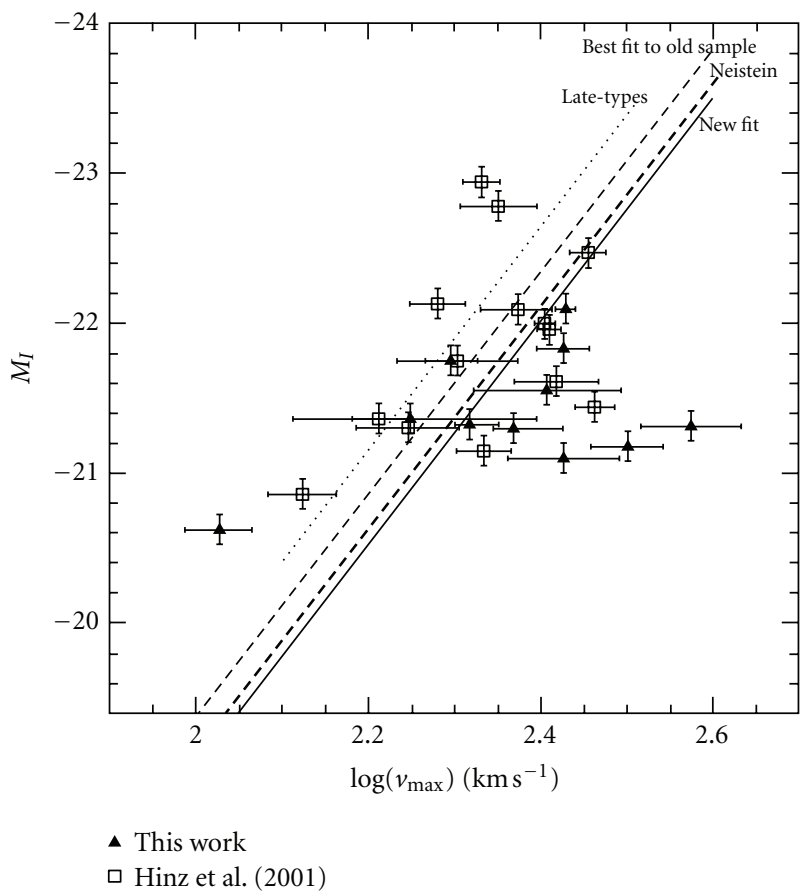

FIgURE 12: Tully-Fisher diagram for S0 Coma galaxies in I-band. The dotted line indicates the TF for late-type spirals and the full line shows the TF of S0 galaxies from the Coma cluster. Credit: Hinz et al. [162]. Reproduced by permission from the AAS.

The location of galaxies in the $V_{c}-R$ plane was analysed by Prieto et al. [168] and Campos-Aguilar et al. [169] for a sample of spiral and S0 galaxies. They found that for a given mass Sc galaxies showed larger angular momentum than Sa galaxies. In contrast, lenticular and irregular galaxies were located at the same position as spiral galaxies in the V-R plane. This result indicates that, dynamically, S0 galaxies are no different from spirals of any type. This could point toward the old idea from van den Bergh [20] that lenticulars and spirals form parallel sequences as shown lately by different groups of researchers [18, 21, 22].

\subsection{Bulge and Disc Interplay}

4.3.1. The Prominence of the Bulge in the Hubble Sequence. Traditionally, the bulge-to-disc-luminosity ratio $(B / D)$ and the bulge-to-total-luminosity ratio $(B / T)$ of galaxies have been used for morphological galaxy classifications. The early work by Burstein [88] shows that for S0 galaxies $B / D>0.3$ (or $B / T>0.75$ ). Some years later, Simien and de Vaucouleurs [86] found that the Hubble classification sequence was mainly driven by the decreasing $B / D$ ratio. In this picture, $\mathrm{S} 0$ galaxies were located between the Es and spirals, as Hubble claimed. Several researchers have used the previous results and have classified their galaxies according to the values of the $B / D$ or $B / T$ parameters. Thus, $\mathrm{S} 0$ galaxies have been considered as those disc galaxies dominated by the bulge component $(B / T>0.6-0.7)$.

Recent surface brightness decomposition of samples of galaxies including $\mathrm{S} 0 \mathrm{~s}$ challenge the previous results. In particular, Balcells et al. [130], using a small galaxy sample (17) including only $4 \mathrm{~S} 0$ s, found no correlation between $B / D$ and the Hubble type but that S0 galaxies has a mean value of $B / D \approx 0.25$. The large $B / T$ dispersion of $S 0$ galaxies have also been observed in other larger samples. Thus, Laurikainen et al. [65] and Weinzirl et al. [73] show that $B / T$ increases from late to early-type galaxies, but that, nevertheless, S0/a galaxies have similar $B / T$ values $(\approx 0.2-0.3)$ to Sa ones. A similar result was obtained by Laurikainen et al. [21], who shows that $\mathrm{S} 0$ galaxies have a wide spread of $B / T$ values. Thus, $13 \%$ of their S0 galaxies have $B / T$ values similar to those for late-type galaxies $(B / T<0.15)$. All these new results show that some galaxies visually classified as S0 have small bulges, as do late-type ones. These galaxies were classified as S0s due to their smooth extended disc, in spite of their bulge prominence. Notice also that galaxy classifications using $B / D$ or $B / T$ could have been misclassified a significant fraction of S0 galaxies.

The large spread found in the $B / T$ values of S0 galaxies could also be in agreement with other results suggesting that spiral and S0 galaxies formed parallel sequences within which early- and late-type galaxies are distinguished by their $B / T$ values (see e.g., [18, 20-22].

4.3.2. Correlation between $r_{e}$ and $h$. The correlation between the bulge effective radius and disc scale length has been extensively studied in the literature (see $[66,94,95,97,103$, 126]). These studies show that larger bulges reside in larger discs. This correlation is shown in galaxy samples including (or not including) S0 galaxies. It has been argued that this relation indicates a link between bulge and disc formation. Barway et al. [170] found that for S0 galaxies the correlation between $r_{e}$ and $h$ depends on the galaxy luminosity. Thus, faint lenticulars show positive correlation between $r_{e}$ and $h$ while this correlation was not present for bright S0s. Nevertheless, this dichotomy was not confirmed by other researches in larger galaxy samples (see [66]).

The relation between the ratio of the bulge effective radius to disk scale length $\left(r_{e} / h\right)$ and the Hubble type has also been studied. Several investigations have found a lack of correlation between those two quantities [94, 95, 130], indicating that the Hubble sequence is scale-free. Thus, Graham and Worley [140] and McArthur et al. [126] found a mean value $r_{e} / h \approx 0.2$ for spirals. In the case of S0 galaxies, the mean values of $r_{e} / h$ reported in the literature goes from 0.2 (see [21]) to 0.36 (see [103]).

4.4. Bar Parameters. The presence of bars in disc galaxies has been extensively studied in the literature. Bars have been identified through visual inspection (e.g., [12, 28, 171, 172]), by analysing the shape and orientation of the galaxy isophotes [118, 173-176], by studying the Fourier modes of the light distribution [113, 177-179]) and by decomposing the surface brightness of the galaxies into their structural components including bars $[21,63,64,70,73,114,116,179,180]$. These studies indicate that bars are observed in optical images of roughly half of all the nearby disc galaxies. This fraction rises to about $70 \%$ when near-infrared images are analysed. 
The fraction of barred galaxies is not constant with the Hubble type. Neither is there agreement on whether the bar fraction is larger in S0 galaxies or in late-type ones. Thus, Aguerri et al. [118] found a bar in $29 \%$ of lenticular galaxies and $\sim 55 \%$ in early- and late-type spirals. In addition, the bar fraction in galaxy discs is higher in those galaxies with bulges with $n<2$ and $B / T<0.2$ (see [73]), suggesting that bars avoid galaxies with large and prominent bulges (see [118]; but see also [171]). This is consistent with the findings by Laurikainen et al. [65] who found that $B / T$ in barred galaxies was smaller than in nonbarred ones. A smaller fraction of bars in lenticulars than in spirals was also found by Laurikainen et al. [66] for a low redshift sample and by Nair and Abraham [28] for a sample of galaxies located at $z<0.1$. Similarly, Barway et al. [181] also found a small bar fraction in lenticulars (15\%), this fraction being a function of galaxy luminosity. Thus, less luminous galaxies have a higher fraction of bars in their sample. Giordano et al. [182] also observed a smaller fraction of bars in S0s than in spirals but, in contrast, less massive galaxies show smaller bar fractions. This was also found by Méndez-Abreu et al. [104] for a sample of bars in the Coma cluster spanning a large mass range. Larger fraction of bars in S0 galaxies, similar to those reported for late-type ones, has been found in other studies (see e.g., [183]).

There are three main observational parameters of bars: length, strength, and pattern speed. I review below the most important results concerning these three parameters for S0 galaxies.

4.4.1. The Bar Length. The bar length has been obtained by eye estimates from galaxy images $[119,184]$, by locating the maximum ellipticity of the galaxy isophotes $[173,175,176]$, by looking for variations in the isophotal position angle $[118,185,186]$ or in the phase angle of the Fourier modes of galaxy light distributions $[115,187]$, or by photometric decomposition of surface brightness distributions [21, 63, $64,70,73,114,116]$. These studies have reported a typical bar length of 3-4 kpc correlated with the disc scale length or galaxy size $[63,173,180]$. The bar length also depends on other galaxy properties such as colour or prominence of the bulge [188].

The bar length normalized by galaxy size depends on the Hubble type. Thus, lenticular galaxies show larger bars than late-type ones $[113,118,186,189]$. Laurikainen et al. [65] also found that the ratio between the bar radius and the disc scale depends on the Hubble type, being larger for S0 galaxies than for late-types.

4.4.2. The Bar Strength. The bar strength represents the contribution of the bar to the total potential of the galaxy. Several methods have been developed to measure it. The most commonly used is the $Q_{g}$ parameter. This parameter estimates the bar torque and was suggested by Combes and Sanders [191]. It has been calculated using different methods (see, [187, 192-194]). This parameter can be accurately estimated by using near-infrared galaxies (see $[65,195$, 196]). Other bar strength measurements are related to the bar ellipticity. This method assumes that bar strength is a function of their ellipticity. Thus, more elliptical bars are stronger than rounder ones (see [197]).

The two methods give similar strength measurements of the bars due to the fact that bar strength computed with bar ellipticities correlates with $Q_{g}$ (e.g.,[198]). Note that bar ellipticity and $Q_{g}$ do not give similar strengths as a function of the Hubble type (e.g., [65]). Nevertheless, Laurikainen et al. [65] showed that S0 bars are weaker than late-type ones using the $Q_{g}$ parameter or the bar ellipticity for measuring the bar strength. In this sense, some studies have shown that bars in lenticular galaxies are weaker than in late-type spirals measuring the bar strength by the $Q_{g}$ parameter (see $[65,190]$ and Figure 13) or by the bar ellipticity [118].

4.4.3. The Bar Pattern Speed. Bar pattern speed is the main kinematic observable in barred galaxies and measures the tumbling pattern of the bar. The pattern speed is related to the so-called corotation radius of the galaxy. This is the radius at which the angular velocity of the disc is equal to the pattern speed of the bar. Barred galaxies are classified according to the distance-independent ratio $R=D_{L} / a_{B}$, where $D_{L}$ is the corotation radius of the bar and $a_{B}$ is the bar semi-axis length. Self-consistent bars cannot exist with $R<1$ (e.g., [199]). Bars showing $1.0<R<1.4$ are called fast bars and those with $R>1.4$ are slow bars.

Several methods have been developed for measuring the pattern speed of bars, including hydrodynamic simulations [201-208] and the shape of dust lanes in the bar regions of some barred galaxies [209, 210]. However, the best way to measure the bar pattern speed of galaxies is through the model-independent method developed by Tremaine and Weinberg ([211]; hereafter the TW method).

The TW method has already been applied to a large sample of S0 barred galaxies [115, 154, 212-216]. All these measurements show that bars of S0 galaxies are fast. According to numerical simulations of Debattista and Sellwood $[217,218]$, strong fast bars are located in galaxies with maximal discs. This means that inside the bar region the baryonic matter dominates. Otherwise, the bar dynamical friction with the dark matter halo of the galaxy slows down the bar efficiently and would produce a slow bar. Other researches claim that the bar pattern speed does not only depend on the mass concentration (see, e.g., [219]). Figure 14 shows the corotation radius versus the bar radius for all galaxies with pattern speed measured using the TW method. This figure shows that, within the error bars, all bars are compatible with being fast (i.e., $R_{\mathrm{CR}} / a_{\mathrm{bar}}<1.4$ ).

The dependence of the bar pattern speed on the Hubble type is not well understood yet. Few measurements of bar pattern speeds for late-type galaxies are available in the literature. Nevertheless, there are some hints that S0 and latetype galaxies show different pattern speed. Thus, Aguerri et al. [178] found a weak correlation between the parameter $R$ and the Hubble type for a sample of ten barred galaxies. They showed that lenticular galaxies have smaller values of $R$ than late-type ones. Recently, Rautiainen et al. [208] have found that all the early-type galaxies of their sample show fast bars, 


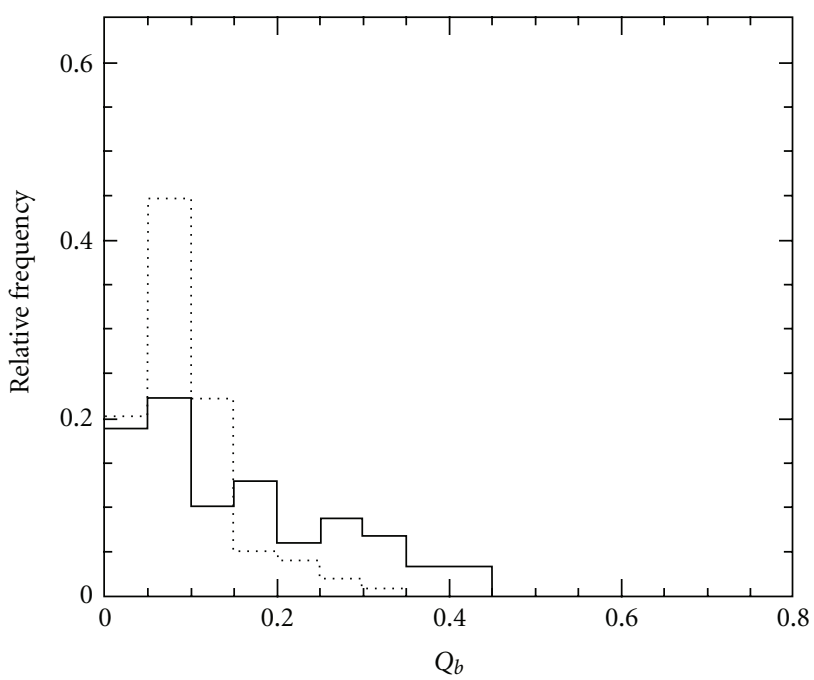

(a)

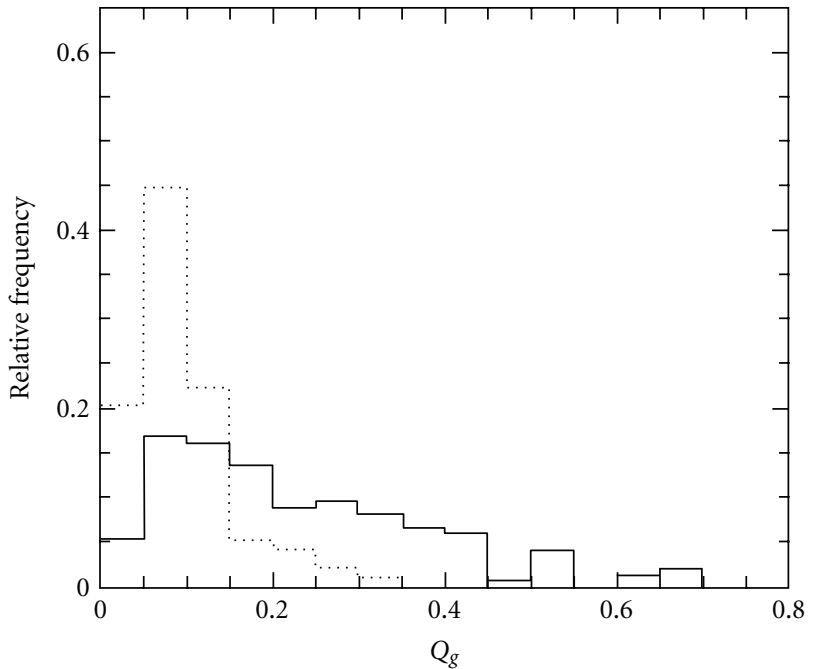

98 NIRSOS S0s - 147 OSUBGS S0/a-Sm

(c)

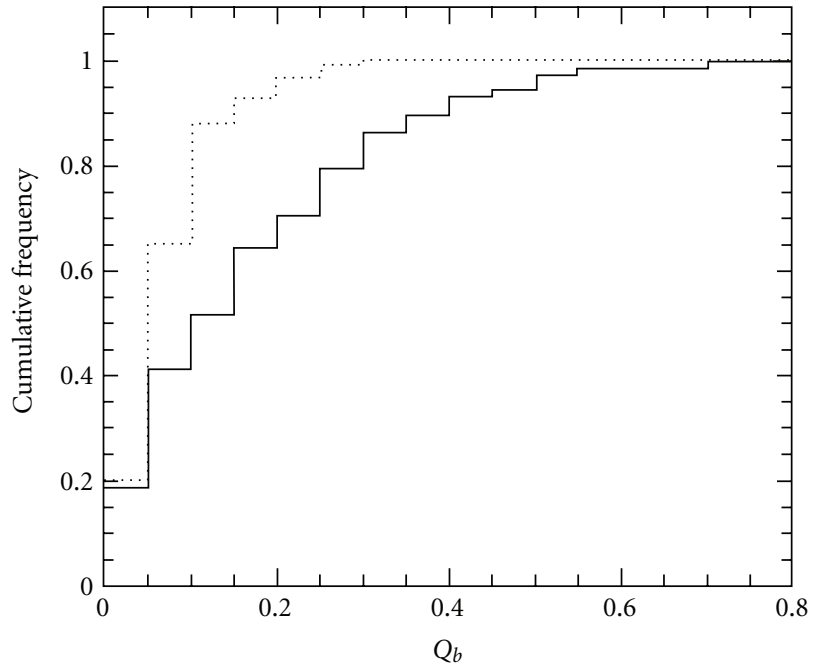

(b)

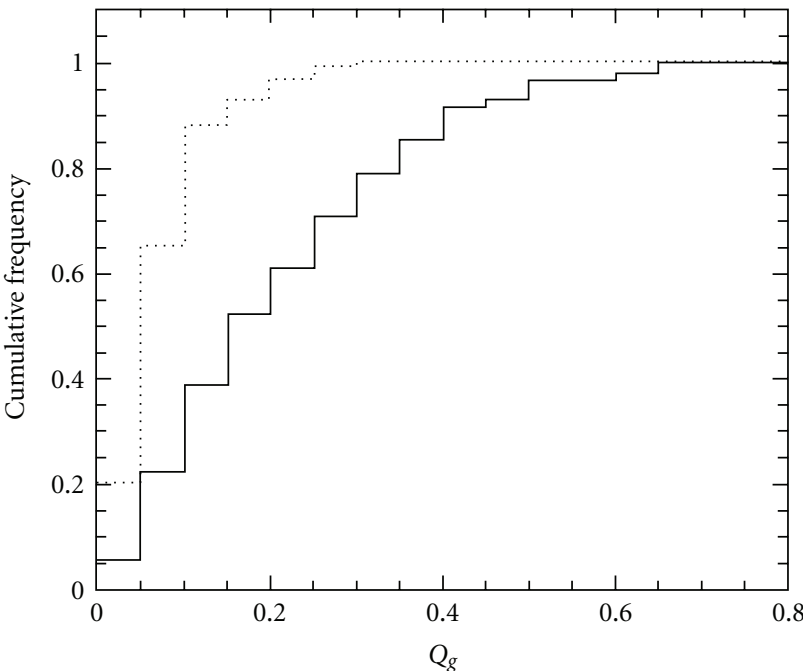

98 NIRSOS S0s

— 147 OSUBGS S0/a-Sm

Figure 13: Histograms of the distribution (a) and (c) and cumulative distribution (b) and (d) of bar strengths in S0 (dotted line) and spiral galaxies (full line). This figure shows that bars are weaker in S0 than in spiral galaxies. Credit: Buta et al. [190]. Reproduced by permission from the AAS.

whereas bars of the late-type galaxies included both fast and slow rotators (see Figure 15).

It is important to notice that all these results concerning bars indicate that discs of late-type and lenticular galaxies are different. Thus, lenticulars show a smaller fraction of bars than late-type ones. In addition, bars in lenticular galaxies are larger, weaker, and faster than late-type bars.

\section{The Case of dSO Galaxies}

Dwarf galaxies are the most numerous type of galaxies in the universe. They are defined as those with low central surface brightness and luminosities below $M_{B}=-18$. According to their colours we can distinguish two main classes of dwarf galaxies: dwarf ellipticals (dEs) and dwarf irregulars (dIs). In general, their surface-brightness profiles can be well fitted by the Sérsic profiles with small shape parameter values $n \approx 1$ $2[102,124,220]$. Nevertheless, there are some dE galaxies with surface brightness profiles that are not well fitted by a single component. Sandage and Binggeli [221] noticed this in some dE galaxies in the Virgo Cluster. Later, Binggeli and Cameron [122] fitted an exponential profile to the outermost regions of the surface brightness of these galaxies, but this profile clearly cannot fit their surface brightness at all radii. These results indicate that these objects have two structural components. These types of dwarf galaxies were called dwarf lenticulars (dS0s). These galaxies are also gas-poor systems like dEs and have evolved with old stellar components but 


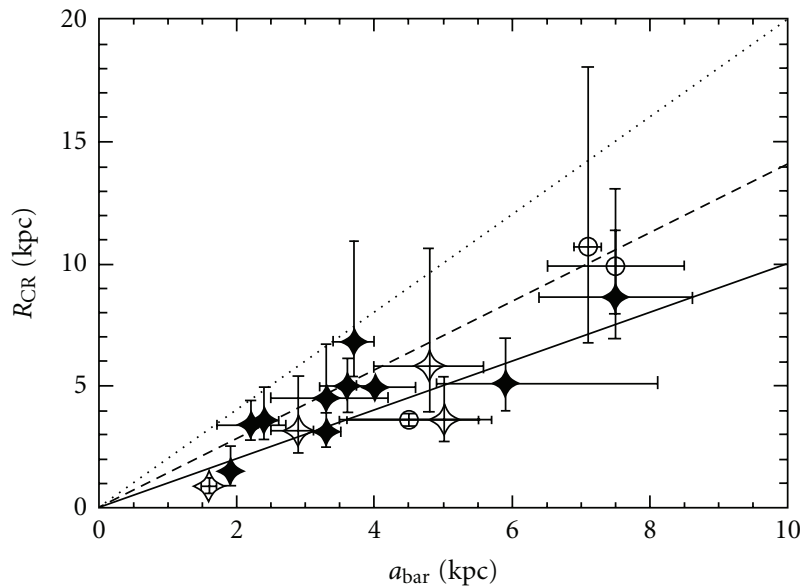

FIGURE 14: The corotation radius $\left(R_{\mathrm{CR}}\right)$ versus the bar radius $\left(a_{\text {bar }}\right)$ for barred galaxies from the literature with bar pattern speed measured by applying the TW method. Full and open stars refer to barred and unbarred lenticular galaxies, respectively. Open circles show spiral barred galaxies. The full, dashed, and dotted lines represent $R_{\mathrm{CR}} / a_{\mathrm{bar}}=1.0,1.4$, and 2.0 , respectively. Fast bars are usually defined as those with $R_{\mathrm{CR}} / a_{\mathrm{bar}}<1.4$ (see text for more details). The data for this figure was taken from [200].

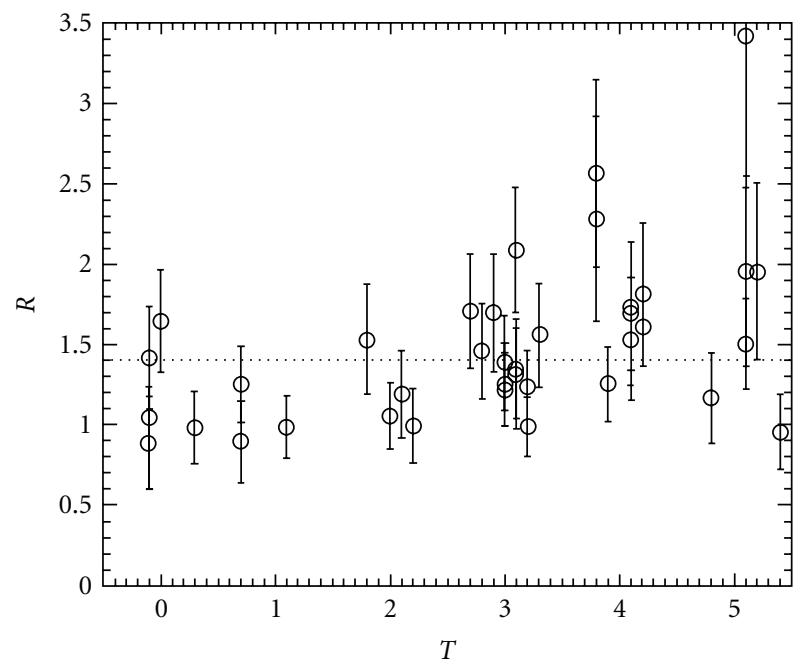

FIGURE 15: The $R=R_{\mathrm{CR}} / a_{\text {bar }}$ parameter as a function of the Hubble type. The horizontal line $(R=1.4)$ represents the separation between fast and slow bars. Credit: Rautiainen et al. [208]. Reproduced by permision from WILEY.

they have higher flattening [222]. Some of these galaxies in the Virgo cluster have disc-like structures due to the presence of bars and/or spiral arms patterns [223-228].

In the Coma cluster, $56 \%$ of the dwarf galaxies in the magnitude interval $-16<M_{B}<-18$ show surface brightness radial profiles well fitted by only one structural component. In contrast, the surface brightness radial profiles of $29 \%$ of the dwarfs in the same magnitude interval cannot be fitted by a single Sérsic component (see [102]).

The structural relations of dwarf galaxies in the Coma cluster were analysed by Aguerri et al. [102], who found that the dS0 population in the Coma cluster show similar structural parameters to those of the dS0s located in the Virgo cluster. In addition, the structural parameters of the disc components in the $\mathrm{dS} 0$ galaxies showed similar relations to the discs of bright spirals. The scale length of the bulges of dS0 galaxies was similar to the scale length of bulges of latetype galaxies. Nevertheless, the mean scale of dEs was found to be higher than that of the bulges of all morphological types of bright galaxies. These results indicate that dSO galaxies are similar to late-type spirals. Thus, dS0s seem to be low-luminosity late-type spiral galaxies (see Figure 16). The dichotomy in the formation between elliptical and spheroidal galaxies has been recently pointed out by other authors (see e.g., [229]).

\section{Formation of Bright and Dwarf Lenticular Galaxies: Different Formation Scenarios}

In the previous sections I have reviewed the properties of the structural parameters of lenticular and spiral galaxies. It should be noticed that the scaling relations shown before give a wide variety of results. Thus, some results indicate that S0 bulges are similar to E galaxies (e.g., same FP, FJ, and size-mass relations, large $n$ Sérsic values), while other scaling relations show that $\mathrm{S} 0$ galaxies are similar to late-type ones (e.g., small values of the $n$ parameter, similar slope of the TF relation but different zero point). This could reinforce the idea of S. van den Bergh that the class of S0 galaxies is formed by galaxies with different origins and evolutions that only share a similar morphological shape. In this section I review the different formation scenarios proposed for lenticular galaxies: I indicate which S0s could be formed by each of these scenarios according to their properties.

6.1. Major Merger Formation. According to the current paradigm of galaxy formation, spheroidal systems (ellipticals and bulges of galaxies) have been formed by major or minor galaxy mergers. In contrast, discs do not survive after major mergers. They were formed by cooling of gas in rotating dark matter haloes. In this paradigm the central bulge of disc galaxies forms prior to the disc as a result of early merging (see the reviews by $[141,230]$ ). In contrast to the bulge, the thin disc forms later from surrounding gas (e.g., [231]).

Galaxy major mergers produce remnants with similar shape and scaling relations to those observed in bulges of S0 galaxies. In particular, the gas content of the progenitors determines the shape and structural parameters of the remnants. Thus, dissipationless numerical simulations of major mergers produce triaxial remnants with a tendency to become prolate systems. In contrast, dissipational remnants are weakly triaxial, but close to oblate [232-236]. Dissipationless numerical simulations of bulge + disc galaxy mergers with different mass ratios produce disc galaxies with $B / T>0.2$ and bulges with a Sérsic shape parameter mainly in the interval $3<n<4$ [237]. In addition, Hopkins et al. [238] has found that high $B / T$ disc galaxies tend to form in major mergers, whereas low $B / T$ objects tend to form from minor mergers. The remnants produced by galaxy 


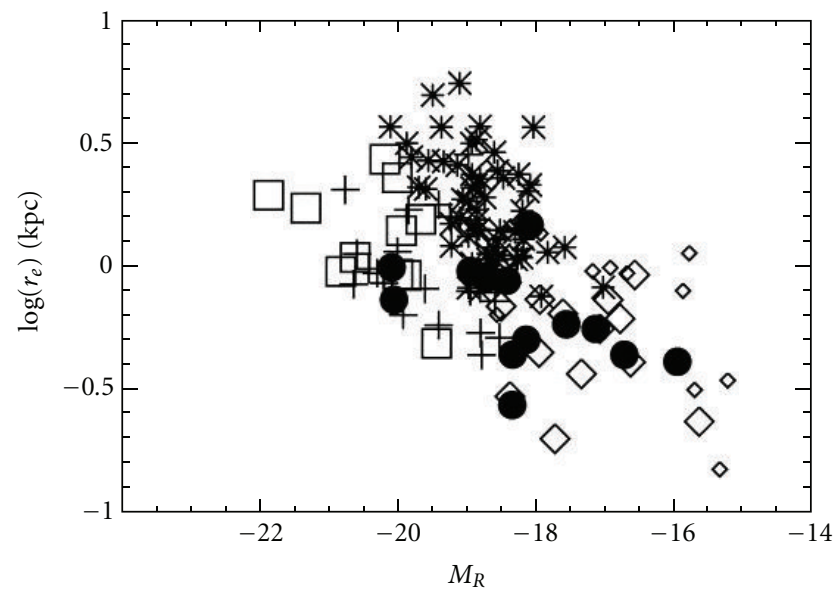

(a)

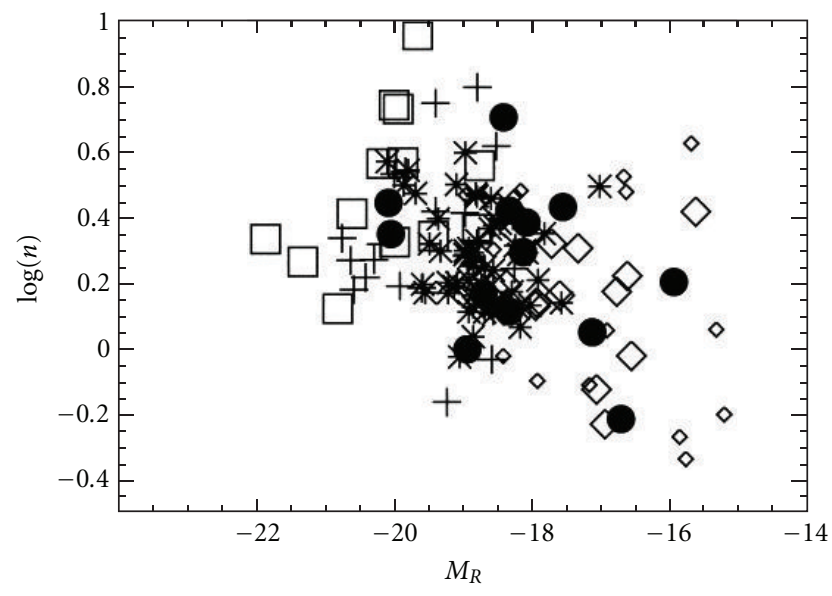

(b)

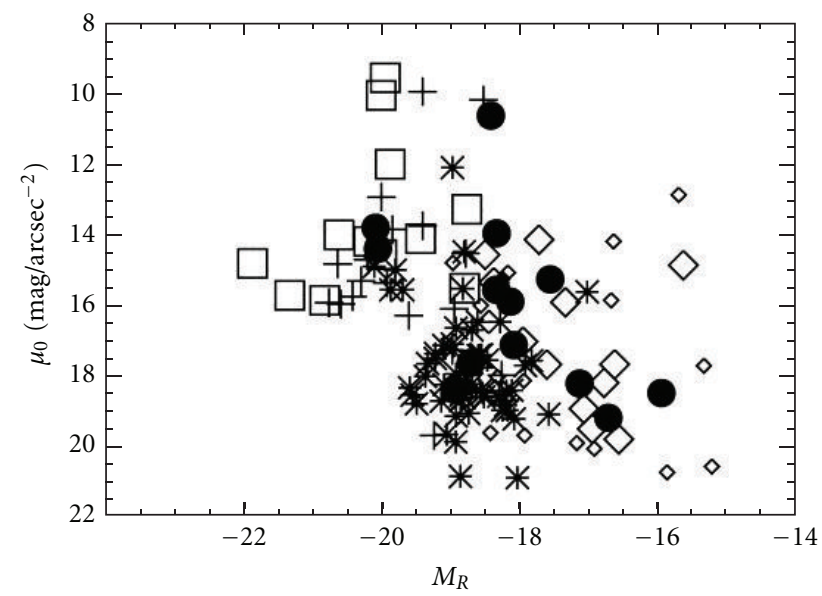

(c)

FIGURE 16: Effective radius (a), Sérsic shape parameter (b), and central surface brightness (c) as functions of the R-band absolute magnitude of dE (asterisks) and bulges of dS0 (diamonds), late-type spirals (circles), early-type spirals (crosses), and S0 galaxies (squares) in the Coma cluster. See for more details [102].

major mergers also follow typical scaling relations to those of elliptical galaxies (see [239]).

The major merger scenario could be applicable to some of the S0 galaxies, especially the most luminous and with prominent bulges $(n>2$ and $B / T>0.3)$. The scaling relations previously shown indicate that the bulges of these S0 galaxies follow the typical scaling relations of elliptical galaxies (fundamental plane, Kormendy relation, size-luminosity relation). In addition, the observed bimodal distribution of the triaxiality parameter shown in a large sample of bulges of S0-Sa galaxies has been compared with the predictions of numerical simulations of spheroid formation (see [104]). In this context, the variety of intrinsic shapes of bulges of S0Sa galaxies with $n>2$ and $B / T>0.3$ required both major dissipational and dissipationless mergers [104].

Recently, it was discovered that a small fraction of nearby E/S0 galaxies show blue colours. These galaxies, called blue ellipticals, constitute only about $10 \%$ of the total population of nearby E/S0 galaxies (e.g., [61, 240-242]). This fraction changes with redshift, constituting up to $30 \%$ at $z \approx 1$ (see [61]). Blue ellipticals with small masses could be the results of merger remnants that triggered the central star formation and built a central bulge component. The gas surrounding this bulge could build a disc on a longer time scale (see [61]). These small blue ellipticals observed at high redshift could be the progenitors of some nearby S0 galaxies.

Major mergers are rare in rich galaxy clusters. They are more frequent in field or galaxy groups. Thus, S0 galaxies formed by this mechanism should be located preferentially in less dense galaxy environments.

6.2. Formation of SO by Satellite Accretion. Satellite accretion must have occurred several times in a disc galaxy over a Hubble time. Mergers of galaxies with similar mass fully destroy the disc and leave remnants similar to E galaxies. In contrast, minor mergers damage the disc less and can produce bulge growth. The growth of the central bulge of the main galaxy depends on the density and relative mass of the accreted satellite. The remnants of these kind of mergers retain significant amounts of rotation (see [244, 245]). Nevertheless, the resulting disc is heated by the 


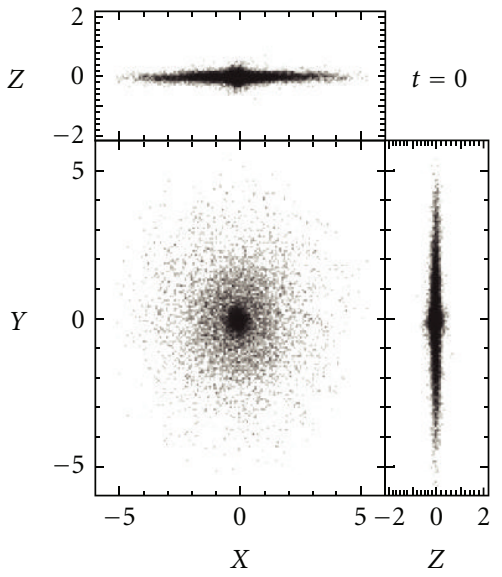

(a)

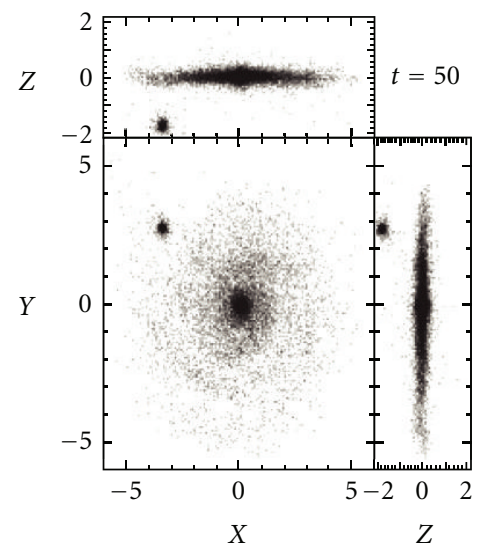

(d)

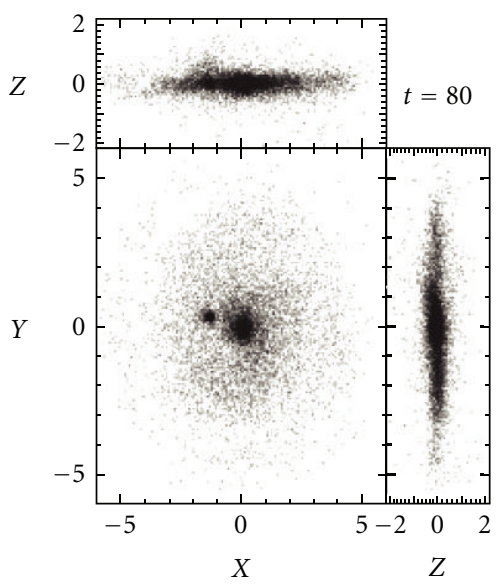

(g)

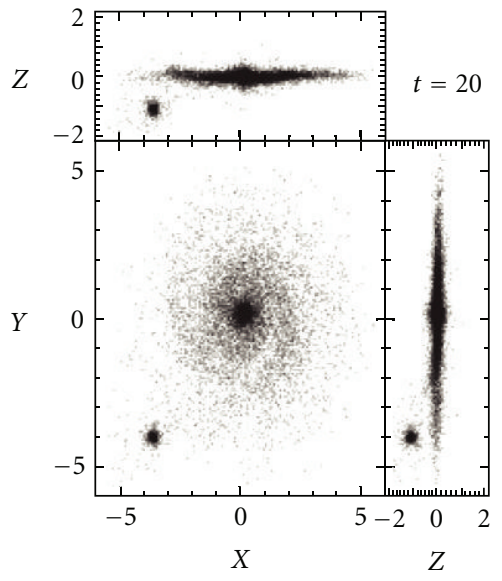

(b)

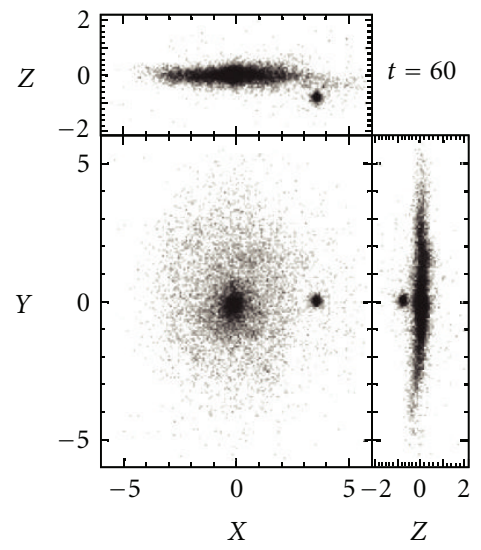

(e)

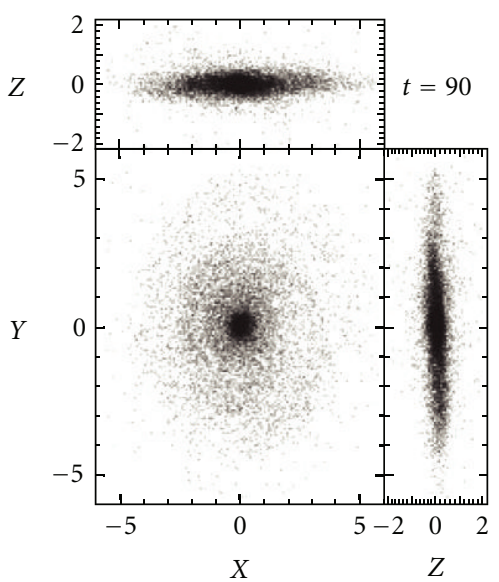

(h)

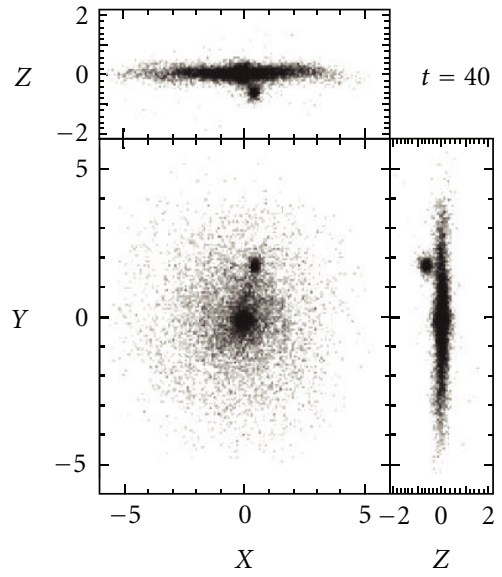

(c)

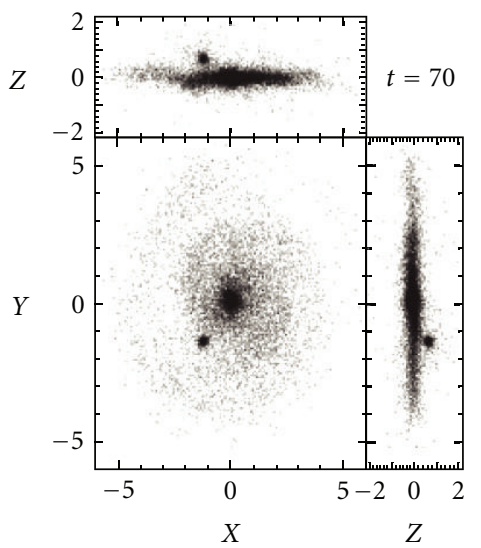

(f)

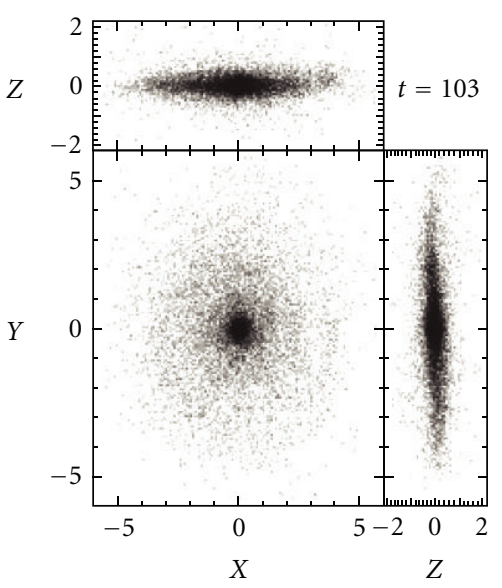

(i)

FIGURE 17: The time evolution of the luminous matter of a minor merger (see details in [243]).

tidal interactions between the satellite and the main galaxy ([246-248]). The final disc is similar to S0 galaxies ([249]). Figure 17 shows the time evolution of the luminous matter of simulated minor mergers. It can be seen that after the merger the main galaxy retains a thick disc (see [243]). The transformation of late-type galaxies by minor mergers was analysed in a series of papers by Aguerri et al. [243] and Eliche-Moral et al. [250].
The most important galaxy transformations were produced by the accretion of dense satellites (see [243]). Dissipationless simulations of minor mergers with different mass ratios and dense satellites show that the accreted satellite can reach the galaxy centre and drive significant growth of the central bulge. The initial conditions of the main galaxy in these simulations were bulge + disc galaxies with an exponential bulge, similar to observed bulges of 


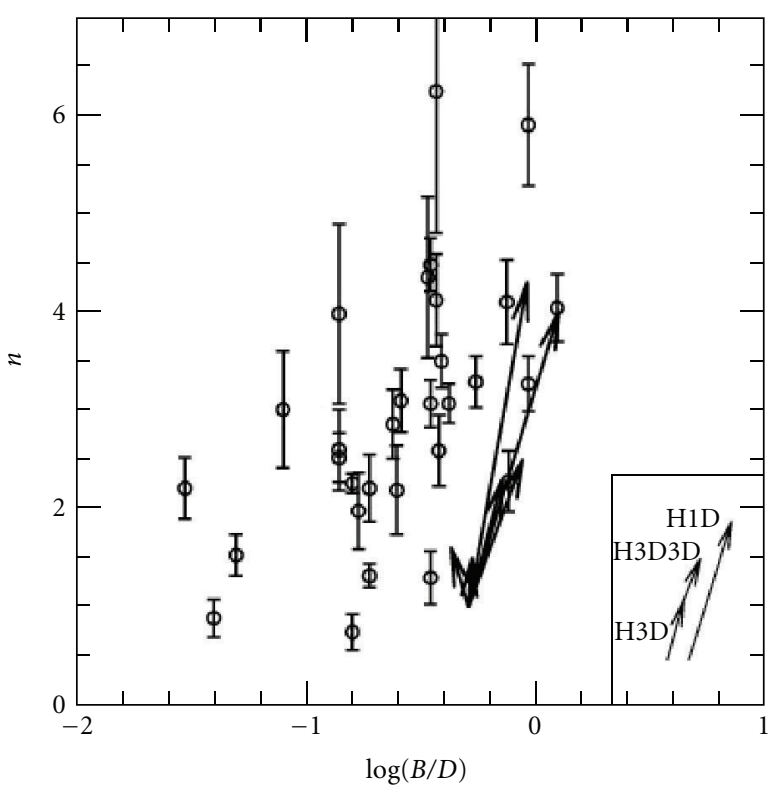

FIGURE 18: Growth vectors of the bulges in the $n-\log (B / D)$ plane for the accretion models by [243]. Each arrow starts at the location of the original model and ends at the $n$ and $B / D$ derived from the twocomponent fit of the surface mass density profile of the models. The points represent the distribution of $n$ versus $\log (B / D)$ for bulges of galaxies from [93]. See more details is [243].

late-type galaxies. After the accretion of these types of satellites the Sérsic shape parameter can rise from 1 to 4 dependent on the mass merger rate. Moreover, the $B / D$ ratio of the final galaxy was in all cases higher than 0.43 , similar to the $B / D$ ratios observed in some $\mathrm{S} 0$ and $\mathrm{Sa}$ galaxies. These simulations show that the efficiency of the process suggests that present day bulges of late-type spirals showing exponential profiles cannot have grown significantly by collisionless mergers. Figure 18 shows the evolution of $n$ and $B / D$ due to the accretion of dense satellites of different masses (see [243]). These simulations also show that S0 galaxies with bulges with large $n$ Sérsic values $n>2-3$ and large $B / D$ ratios can be formed by the transformation of latetype galaxies through minor mergers (see [243]).

The accretion of less dense satellites was studied by Eliche-Moral et al. [250]. The primary and secondary galaxies were spiral galaxies with their particles distributed in bulge, disc, and halo components. Both galaxies follow the Tully-Fisher relation similar to real ones. This scaling means that the material of the satellites did not reach the galaxy centre but was instead distributed through the disc of the main galaxy. Thus, particles from the satellite disc deposit their mass onto the primary disc. In contrast, particles from the satellite bulge settle into a rather cold inner disc or ring (see also Eliche-Moral et al. [251]). In addition, an inward flow of material from the disc to the bulge region is produced during the satellite accretion due to the presence of transitory nonasymmetric distortions in the main disc. The satellite accretion drives the initial main galaxy towards an earliertype secular evolution. In particular, the primary galaxy evolves toward higher $B / D, n$, central velocity dispersion, scale length of the disc, and lower central surface brightness of the disc. In particular, $n$ increases by a factor of $\approx 2.3$ and $B / D$ doubles its initial value (see Figure 19 ).

In both cases the accretion of high- and low-density satellites hits the thin disc of the primary galaxy. This vertical heating of the disc depends on the orbit and mass of the satellite. The thick disc created by these types of mergers is reminiscent of the thick discs seen in S0 and early-type galaxies. Bekki [252] and Bournaud et al. [253] also showed that unequal-mass gas-rich spirals (mass ratio $\approx 0.3$ ) can also produce remnants similar to S0 galaxies. The mergers produce an enhanced star formation, thicken the disc of the main galaxy, and produce a subsequent transformation of the main galaxy into an S0 type galaxy. This formation mechanism has been proposed in order to explain the formation of some S0 galaxies [254, 255].

The minor accretion models proposed by Aguerri et al. [243] and Eliche-Moral et al. [250] have certain limitations and caveats that should be discussed. Neither model includes gas hydrodynamics or star formation. We may expect some changes in the dynamics of the remnants due to gas concentration in the galaxy nucleus. This could produce changes in the $M / L$ of the central regions of the galaxies and changes in some structural relations. It is also important to notice that dense satellites easily produce counterrotating bulges in the primary galaxy when the satellite has retrograde orbits. This is an important limitation because counterrotating bulges are not very common in galaxies (see [256]). This is not the case in the simulations with less dense satellites [250]. Nevertheless, in this case the Sérsic shape parameter only grows from $n=1$ to $n \approx 2-2.5$. Thus, lenticular galaxies with $n \approx 4$ and large $B / D$ cannot be explained.

6.3. Evolution of Galaxies in High-Density Environments. Since the first observations of galaxies in clusters it has been well known that the galaxies located in high-density environments have different properties from those located in the field [257-259]. Early studies claimed that galaxies in clusters and the field were different because they were born differently. Today, it is thought that these differences could be due to the evolution of galaxies being dependent on the environment where galaxies live (e.g., [260] But, see also [18]).

One of the differences between galaxies in clusters and the field is morphology. In dense environments the fraction of ellipticals and S0 galaxies is larger than in the field. In contrast, the fraction of spiral and irregular galaxies is higher in less dense environments. This is the so-called morphologydensity relation observed in nearby $[261,262]$ and high redshift [263] clusters. Figure 20 shows the morphologydensity relation for a sample of nearby galaxy clusters (see [262]). This morphological mixing is not constant with redshift. Thus, it was observed that the S0 population of galaxies is smaller in more distant clusters. This decrement is compensated by an increase in the spiral population (see $[263,264])$. The morphology-density relation has been one of the strongest arguments in favour of the existence of an evolutionary trend between spiral and lenticular galaxies. In 


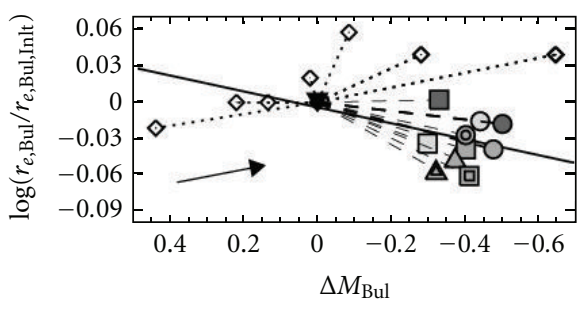

(a) NS

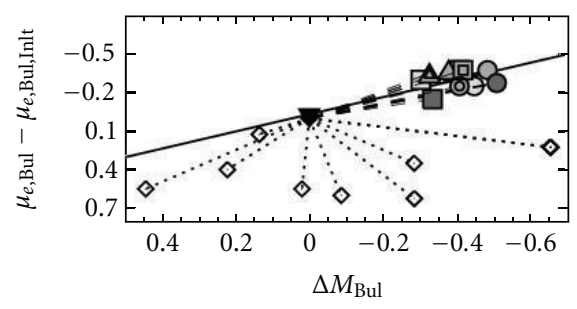

(b) NS

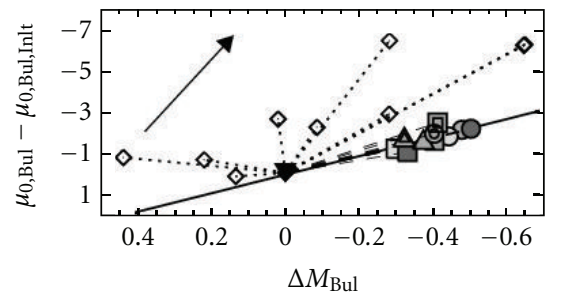

(c) $\mathrm{S}$

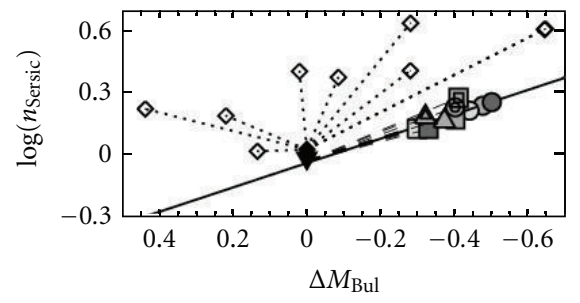

(f) $\mathrm{S}$

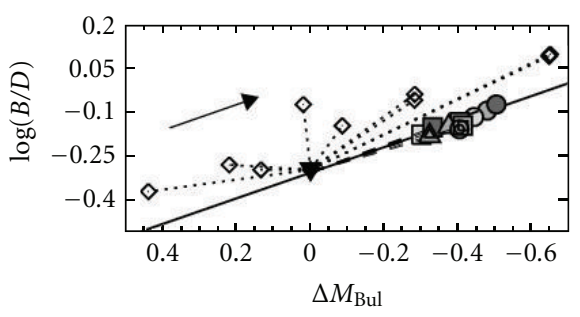

(i) $\mathrm{S}$

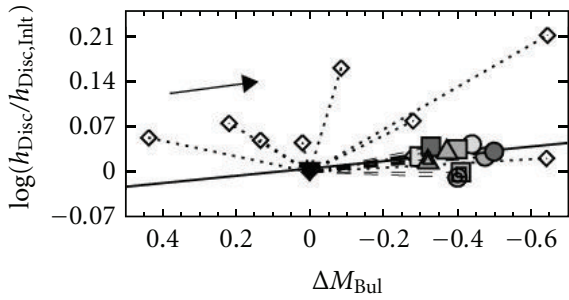

(d) NS

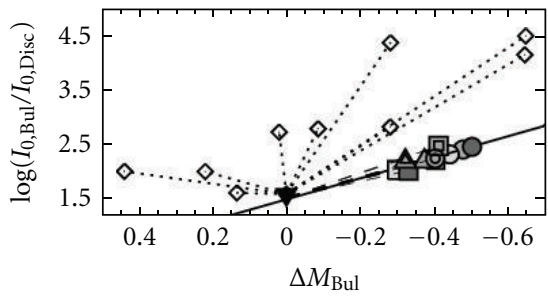

(g) $\mathrm{S}$

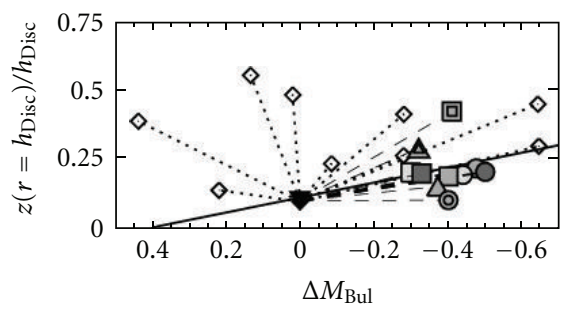

(j) NS

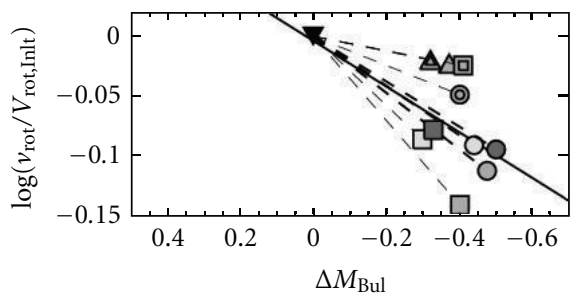

(1) NS

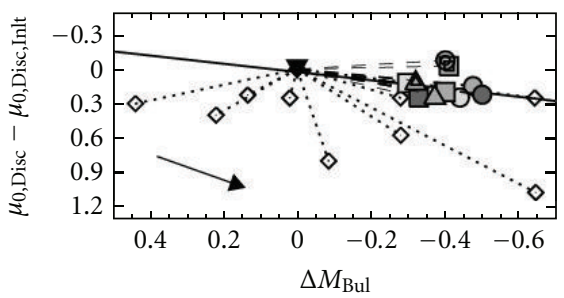

(e) NS

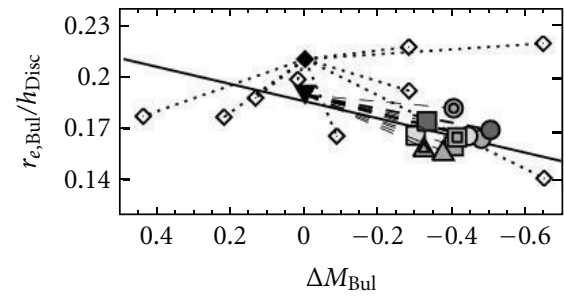

(h) NS

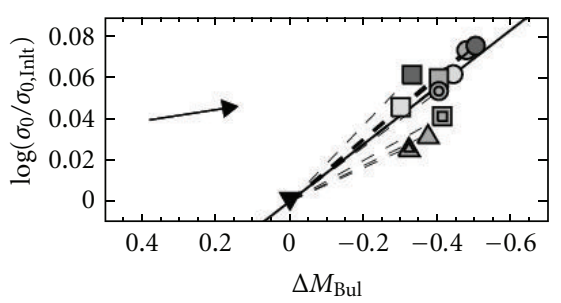

(k) $\mathrm{S}$

Figure 19: The dependence of bulge and disc parameters, and $B / D$ ratio, on the increment of the bulge magnitude (see details in [250]).

this picture, S0 galaxies are descendent from spiral galaxies that have stopped their star formation due to environmental processes.

Galaxies in high-density environments evolve through many different physical processes. We may mention harassment [260], ram pressure stripping [265-267], tidal effects and mergers [268, 269], or starvation [267, 270]. I review below the morphological galaxy transformations produced by these environmental processes.
6.3.1. Gas Stripping. X-ray observations of galaxy clusters reveal the presence of a large amount of hot gas $\left(T \approx 10^{7}-\right.$ $10^{8} \mathrm{~K}$ ) in the intracluster medium. This hot gas contains most of the baryons of galaxy clusters. The motion of the galaxies through this hot intracluster medium produces a ram pressure that can strip a significant fraction of the gas content of galaxies in clusters (see $[265,266])$. This ram pressure is proportional to $\rho_{\mathrm{ICM}} v_{\text {gal }}^{2}$, where $\rho_{\mathrm{ICM}}$ and $v_{\text {gal }}^{2}$ are the gas density of the intracluster medium (ICM) and the 


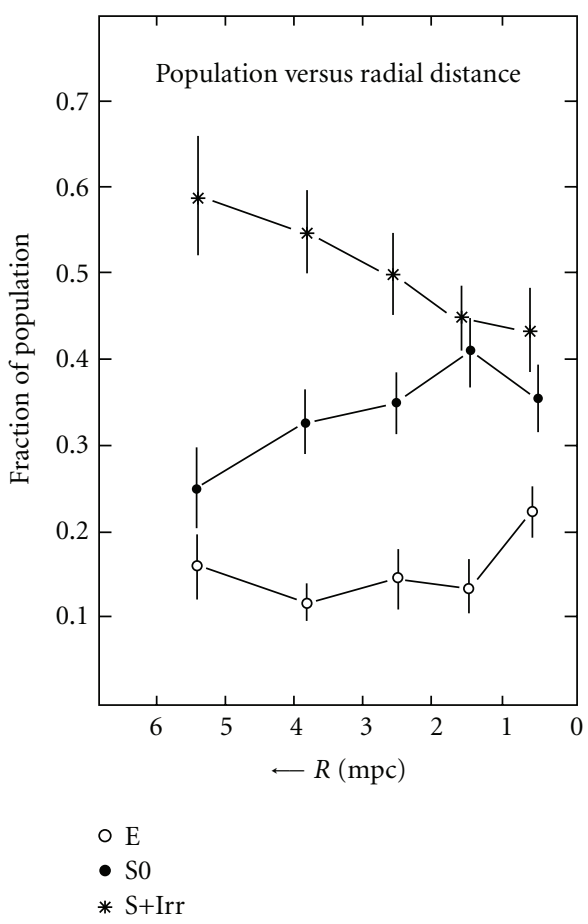

(a)

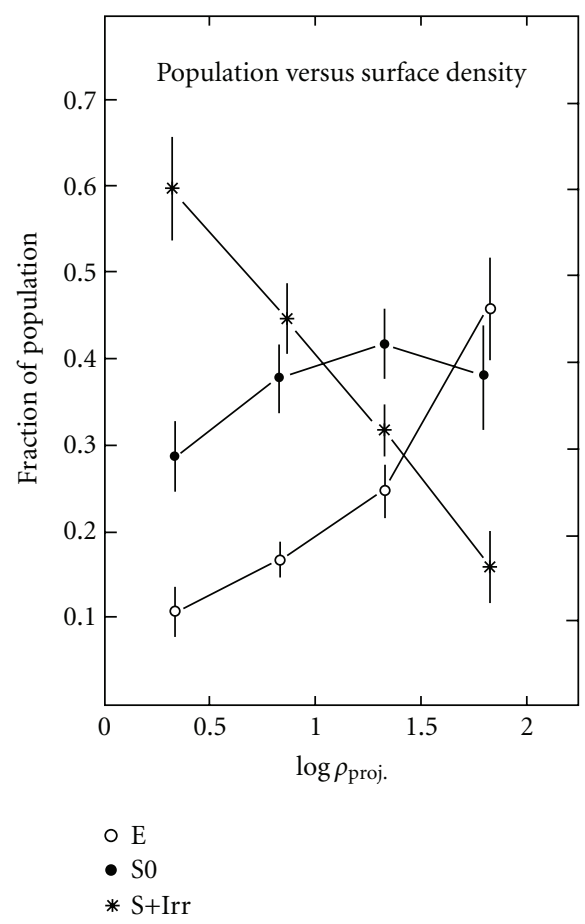

(b)

FIGURE 20: Galaxy population gradients in galaxy clusters as a function of radial distance from the cluster center (a) and local galaxy density (b) Credit: Dressler [262]. Reproduced by permission from the AAS.

relative velocity between the galaxy and the ICM. This effect is thus stronger in the central regions of massive clusters where the gas density and the relative velocity of the galaxies are higher.

The efficiency of the stripping depends on several properties of clusters and galaxies, such as the location of the stripped gas in the galaxy, the masses of the host cluster and galaxies, and the density of the gas. The gas in galaxies can be found as cold or hot gas. The cold gas is located inside galaxy discs, while the hot gas is located in the galaxy halo. Both types of gas can be stripped from galaxies. The gas from galaxy haloes can be stripped more easily than gas from the discs. The required relative velocity between the galaxy and cluster and the gas density of the intracluster gas are thus significantly much lower than those from disc stripping (see [267]). The gas stripping also depends on the mass of the host cluster and the mass of the galaxy. Stripping is more efficient in more massive clusters and in less luminous galaxies [267, 271, 272]. The stripping also depends on the density of the gas inside galaxies. Thus, low-density gas is more quickly stripped at all galaxy radii than high-density gas.

Gas stripping has been observed in several bright dwarf galaxies located in nearby galaxy clusters (e.g.,[273-279]). It is also supported by the HI deficiency observed in some spiral galaxies in clusters [280-284]. Both disc and halo gas stripping produce similar effects. This is the truncation of the star formation and reddening of galaxy discs producing strong morphological transformations. Thus, late-type spirals can be transformed into lenticular ones (see [270] and
Figure 21). The gas stripping and the stopping of the gas accretion onto the disc of the galaxies also stop the formation of bars in galaxies. In addition, it is also expected that gas stripping does not produce significant disk heating. This mechanism could explain several observational properties of lenticular galaxies such as the observed low values of $n$ and $B / T$ for some lenticular galaxies, the lower fraction of bars in S0 galaxies, the different Tully-Fisher relation shown by $\mathrm{S} 0$ galaxies, and the specific number of globular clusters observed in S0 galaxies (e.g., [21, 22, 118, 167, 285]).

6.3.2. Galaxy Interactions. Galaxies in high-density environments like galaxy clusters can suffer several gravitational interactions with a wide variety of impact parameters, relative velocity between galaxies and mass ratios. Interactions in rich galaxy clusters are expected to be more abundant and faster than in small galaxy groups. The harassment scenario proposes that the cumulative effects of fast tidal encounters between galaxies and with the gravitational potential can produce dramatic morphological transformations in galaxies. Thus, bright galaxies can lose a significant fraction of their stellar and gas content. The fraction of mass lost can be high enough in order to transform bright galaxies into dwarfs ([260, 286-290]).

Observational indications of this mass loss have been found for the bright spiral galaxies located in the Coma cluster. Thus, Aguerri et al. [101] and Gutierrez et al. [100] found that the scale lengths of bright spiral galaxies in the Coma cluster were significantly smaller than those for spirals with similar luminosities located in less dense environments. 


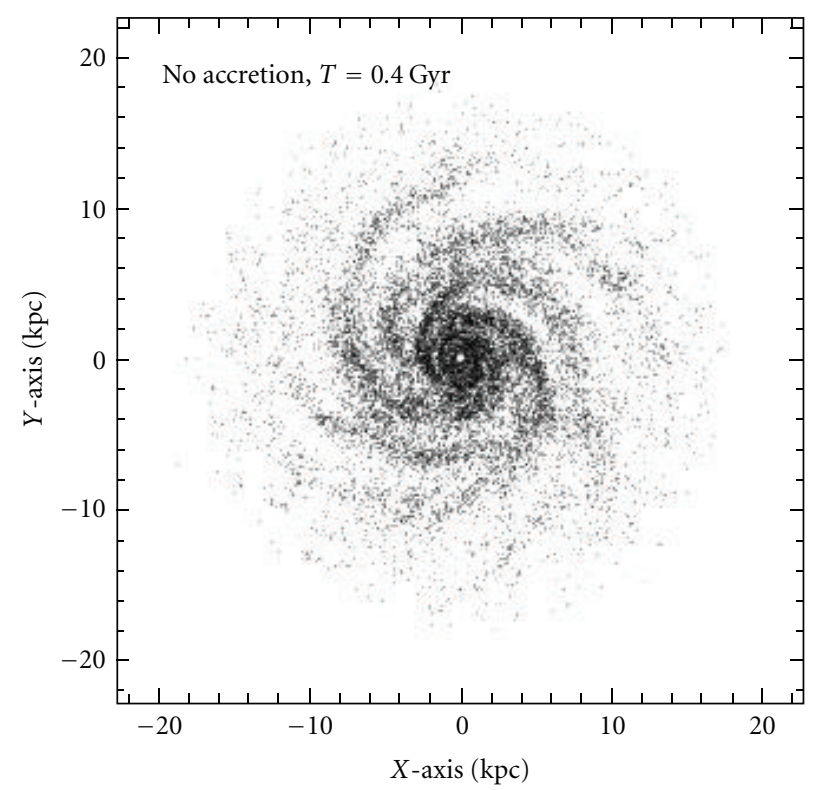

(a)

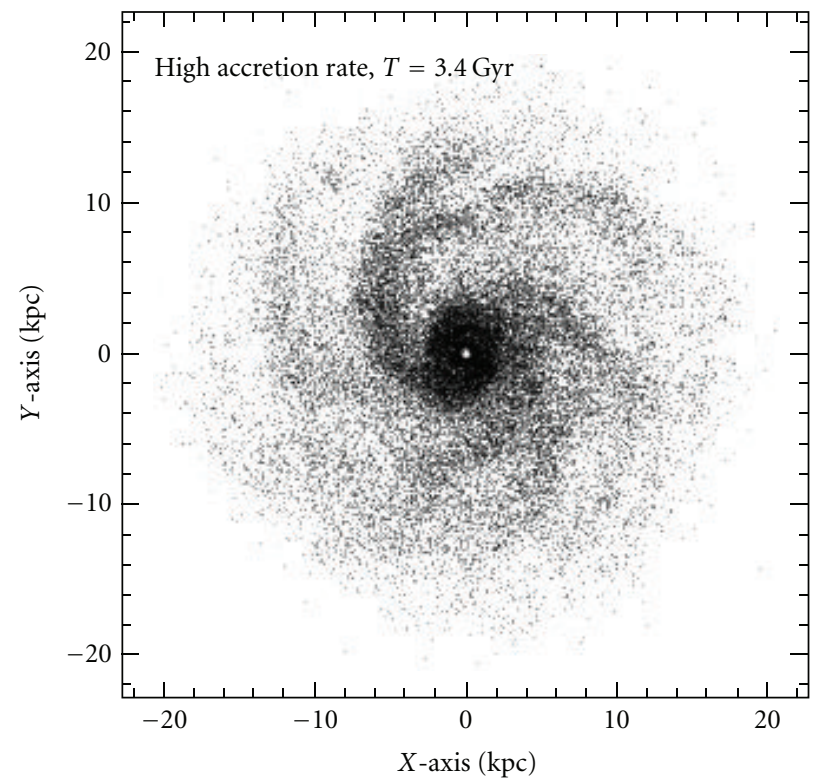

(c)

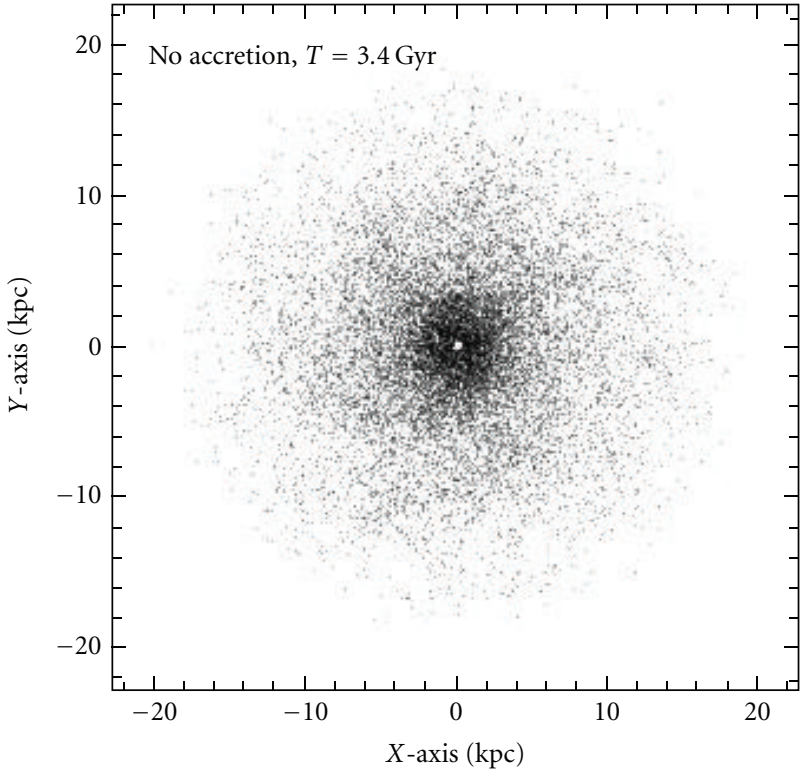

(b)

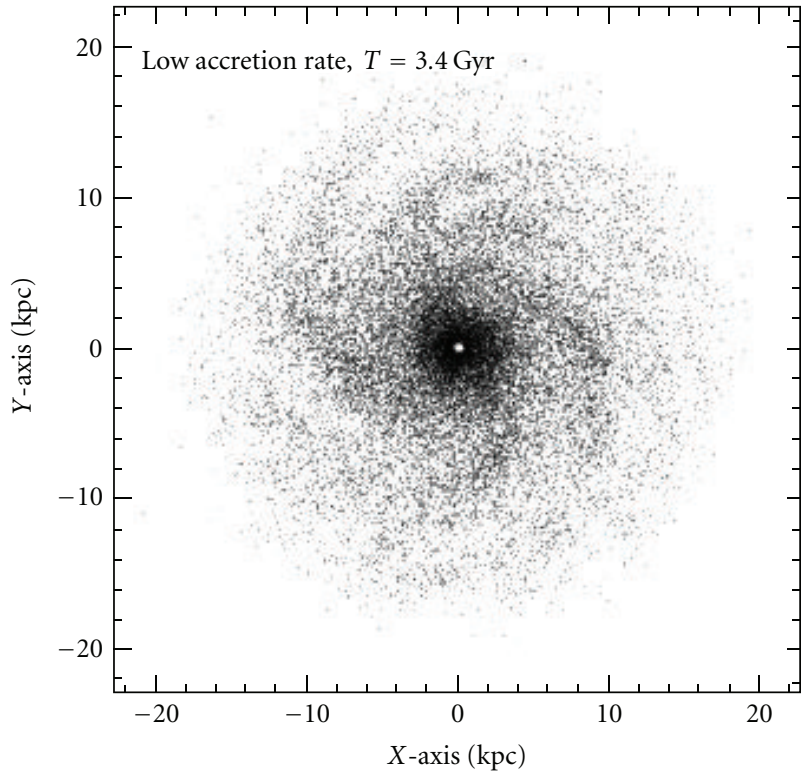

(d)

FIgURE 21: Morphological properties for different models from [270] with no gas accretion at $T=0.4$ Gyr (a) and $T=3.4$ Gyr (b) Models with high (c) and low (d) gas accretion rates. Credit: Bekki et al. [270]. Reproduced by permission from the AAS.

The disrupted stars could form part of the so-called diffuse light detected in some nearby galaxy clusters such as Virgo [291-296], Coma [297, 298], and Fornax [299].

Mastropietro et al. [288] and Aguerri and GonzálezGarcia [289] have studied the properties of the remnants after several fast tidal encounters. The initial conditions of the simulations done by Aguerri and González-Garca [289] consist of disc galaxies with different $B / T$ ratios. These initial systems undergo several tidal interactions, transforming the initial spiral galaxy into a dwarf-like system. The simulations show that fast tidal interactions are very efficient mechanisms for removing stars and dark matter particles from galaxies, especially from the disc and halo components. In contrast, the bulge particles were not removed. After four or five tidal interactions, the initial galaxy has lost up to $80 \%$ of its total mass (see Figure 22). The kinematic analysis of the remnants shows that some of them still conserve an external rotating disc. In contrast, other remnants are systems supported by pressure. Nevertheless, the scale length of the resulting disks is about $40-50 \%$ shorter than the initial ones. In addition, the tidal interaction easily creates tidal features in the discs such as stable bars.

The final position of the resulting galaxies in the fundamental plane depends on the initial conditions of the simulations. Thus, models with initially large $B / D$ ratios are closer to the face-on view of the fundamental plane defined 


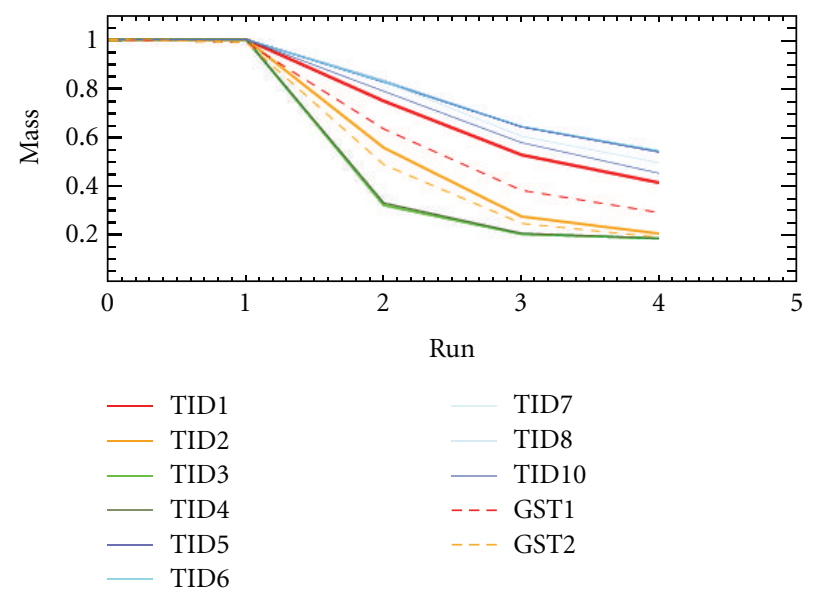

(a)

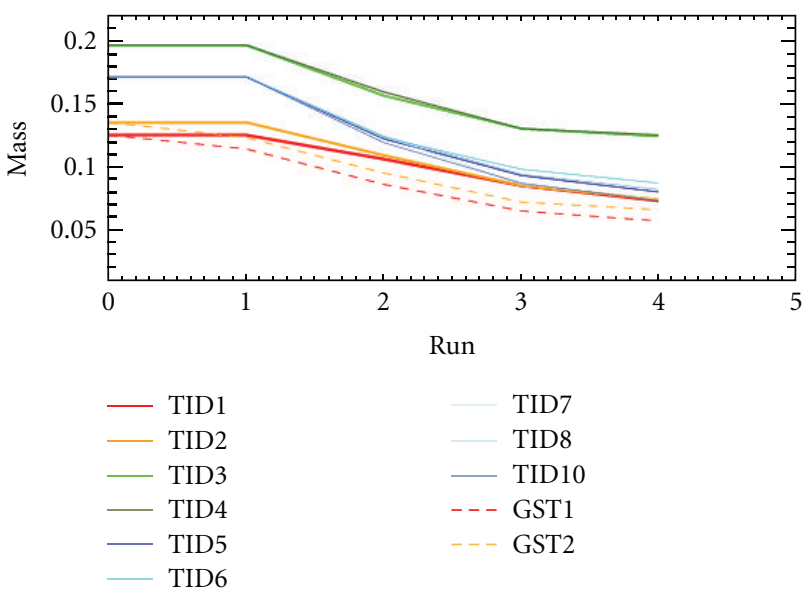

(b)

FIgURE 22: Change of total (a) and luminous (b) mass of simulated galaxies after different fast tidal events (see details in [289]).

by bright Es and bulges of early-type galaxies. In contrast, galaxies with initially small $B / D$ ratios are located at the position of the dwarf galaxies in the face-on view of the FP. These simulations show that $\mathrm{dE}$ and $\mathrm{dS} 0$ galaxies can be formed by the tidal disruption of late-type spirals. In contrast, the tidal disruption of early-type galaxies would produce a final galaxy similar to elliptical or S0 galaxies.

One of the most prominent features that tidal interactions induce in the discs of galaxies is the presence of stable and prominent bars (see $[288,289]$ ). Although the properties of these bars are similar to those observed in some dwarf galaxies, bars are not common in dwarf galaxies (see [172]). This bar formation is one of the caveats of these simulations and could be related to their initial conditions. Other initial conditions of these models should be taken into account, in particular initial models with thicker discs, which could reduce the bar formation (see [300]).

Aguerri and González-Garcia [289] have analysed the correlations between certain structural parameters of the remnants. In particular, they analysed the correlation between Sérsic parameters $r_{e} / h$ and $n$ observed in spiral galaxies (e.g., [94, 103, 125, 126, 128, 129]). Thus, those bulges from spiral galaxies with larger values of $r_{e} / h$ also show higher $n$ values. The simulated dwarf objects formed by fast tidal interactions do not follow the trend followed by real galaxies. Thus, for a fixed value of $n$, tidally formed dwarf galaxies have larger $r_{e} / h$ ratios. This is because discs were strongly truncated due to tidal encounters. In contrast, the effective radius of the bulges does not change. The search for outliers in the $r_{e} / h$ versus $n$ relation could provide dwarf systems (dS0s) formed through the fast interactions of disc galaxies.

It is well known that early-type galaxies follow the luminosity-metallicity and velocity dispersion-metallicity relations (e.g., [301]). Thus, more metal-rich galaxies have higher central velocity dispersions and are more luminous. Tidal stripping should not change the metallicity of the galaxies. This would imply that low-mass systems formed by tidal stripping of bright disc galaxies could well deviate from the luminosity-metallicity relations displayed by bright galaxies. This point is still not well analysed in the literature. Nevertheless, some approaches have recently been undertaken. For example, it has recently been observed that some dwarf galaxies are located outside to the metallicityluminosity relation (e.g., [302]).

6.3.3. Cluster Tides. Galaxies in high-density environments do not only interact with one another but also interact with the cluster gravitational potential. Thus, cluster and/or group tides can also produce strong morphological transformations in spiral galaxies. The tidal field of the clusters as a whole can trigger cluster spiral, nuclear, and disc activities. This effect is more important near the centre of clusters or groups. A higher fraction of starburst galaxies might be expected in the cores of clusters or groups of galaxies. The long-term effects of this activity would be to clear the gas from disc spirals and changing them into S0 galaxies [303, 304]. Semianalytic models suggest that tidal fields can produce a segregation of galaxies by mass in clusters $[305,306]$. The evolution of the galaxies inside clusters is determined by their trajectories and due to the substructure presented in galaxy clusters. These tidal interactions are insufficient to transform a spiral galaxy into an elliptical one. Nevertheless, they can produce the transformation of spiral galaxies into S0s [307].

Evidence for the morphological transformation of galaxies by the cluster environment has been observed in the S0 population of galaxies in the Coma cluster. Poggianti et al. [308] studied the stellar populations of elliptical and S0 galaxies in the Coma cluster. They found that a large fraction of the $\mathrm{S} 0$ population has undergone star formation during the last $5 \mathrm{Gyr}$, not observed in elliptical galaxies. They concluded that the $\mathrm{S} 0$ galaxies with recent star formation are the descendants of star-forming spirals whose star formation has been halted due to the effect of the cluster environment. Nevertheless, the Coma cluster could be an exception to the fact that most of the morphological transformations in clusters since $z=1$ is not confined to massive clusters. On the contrary, it was most pronounced in low-mass clusters (see [309]). 
6.3.4. Environmental Tides. The picture of galaxies falling into static galaxy clusters is very simplistic and far removed from reality. In the hierarchical structure formation theory dark matter haloes grow through the accretion of smaller units. This formation is scale-free. Thus, clusters and groups of galaxies grow through the accretion of smaller groups. Optical and X-ray studies of galaxy clusters show that a large fraction of them have recently accreted other groups or clusters [310-314]. This can be seen in the substructure detected in some nearby galaxy clusters (see [315] and references therein).

Mergers of galaxy clusters could enhance star formation, as suggested by numerical simulations (e.g., [316]) and is claimed to have been seen in some observational studies [317, 318]. These mergers therefore produce strong gravitational tides acting across galaxies and transforming spiral galaxies into S0s [307, 319]. Nevertheless, it is not easy to observe a cluster merger. The transformation of galaxies due to cluster mergers could depend, among other considerations, on the phase in which the merger is observed, or on the viewing angle. There are very few well-identified examples of cluster mergers in the literature. They have been identified by a clear segregation between the dark matter and the intracluster gas. These mergers are the bullet cluster [320, 321], Abell 520 [322], and MACS J0025.4-1225 [323]. This last cluster has recently been studied by Ma et al. [324], who found a large fraction of $\mathrm{S} 0$ galaxies and interpreted this result as a transformation of spiral galaxies into S0s due to ram pressure and/or tidal forces during the cluster merger.

6.4. Passive Evolution of Spiral Galaxies. The distribution of galaxies in colour-magnitude and colour-mass diagrams shows that they are located in two main regions: the blue cloud and the red sequence. Study of the colour-magnitude diagram of galaxies at different redshifts indicates that galaxies stop their star formation and pass from the blue cloud to the red sequence. Moreover, this migration is strongly mass dependent. Thus, more massive galaxies reach the red sequence at higher redshift than less massive ones (e.g., $[325,326])$. The fact that galaxies are not homogeneously distributed throughout the colour-magnitude diagram indicates that galaxies pass from the blue cloud to the red sequence on short time scales.

The reddening of spiral galaxies could produce S0s by fading the spiral arms of the progenitors. This reddening could be due to processes related to the galaxy environment, or they could be processes related only to the internal galaxy evolution. One of the internal processes that could regulate the star formation in galaxies is feedback processes heating the cold gas in galaxies and stopping their star formation. Dekel and Silk [327] proposed that feedback from supernovae could stop the star formation in galaxies and drive their evolution. This feedback scenario could drive the star formation history and evolution of dwarf galaxies, but it cannot heat the gas of massive haloes and stop their star formation. Such additional heating could come from feedback of active galactic nuclei (AGN; see [328]). Thus, outflows and jets from the central supermassive black holes of galaxies are expected to heat the cold gas and stop further star formation in the galaxies (e.g., [329-331]). In a series of papers, Schawinski et al. [332, 333] proposed that AGN feedback is mainly responsible for stopping the star formation in star-forming early-type galaxies. This process would transform early-type spiral galaxies located in the blue cloud into galaxies located close to or in the red sequence. These transformed early-type galaxies could be the progenitors of later S0 galaxies. This mechanism would produce S0 galaxies with similar properties to those of earlytype spiral galaxies.

The red sequence is not only formed by ellipticals and early-type galaxies. It has recently been discovered that a small fraction of late-type spirals (about 6\%) show red colour and are located in the red sequence (see [334]). The red colours of these late-type galaxies is not due to large dust extinction. In addition, these galaxies are mainly located in intermediate galaxy density environments. This implies that the environment alone has not produced the reddening of these galaxies. Masters et al. [334] have also demonstrated that these red spiral galaxies are not recent poststarbursts. Rather, their star formation has ceased gradually. They found that most red spirals (about 70\%) exhibit bars. They argued that the cessation of the star formation in these galaxies should be related to the presence of bars. These passive spirals could be precursors of some S0 galaxies (see also [335]).

These two internal processes (AGN feedback and passive evolution) could explain the similarities of SOs and spiral galaxies. The S0 galaxies formed by these mechanisms should be in intermediate or less dense galaxy environments. However, these internal processes could not explain the different bar properties observed between spiral and lenticular galaxies: different bar fractions (e.g, $[66,118,182]$ ) or pattern speed (e.g., $[178,194,208])$.

\section{Conclusions}

In this paper I have reviewed the observational properties of the photometric components of lenticular galaxies. I have selected samples of galaxies containing S0s and with structural parameters obtained in a homogeneous way by multicomponent surface brightness decompositions. The properties of the different components show that some lenticular galaxies are similar to ellipticals whereas others show similar relations to spiral galaxies. In particular, the most luminous S0s with $B / T>0.3$ and $n>2$ follow similar relations to elliptical galaxies. In contrast, less luminous lenticulars with $B / T<0.3$ and $n<2$ have structural relations closer to spirals. These properties could indicate that the class of lenticular galaxies could be formed by galaxies with different origins and evolutions with only the shape as the common link.

I have reviewed the different formation mechanisms proposed in the literature for understanding the formation of lenticular galaxies. There is a large variety of formation mechanisms that produce S0s over very different time scales. Each of these mechanisms produces S0 galaxies with different properties. Thus, lenticular galaxies with high $n$ and $B / T$ 
values should be produced by one major or several minor mergers or passive evolution of early-type galaxies. Those S0 galaxies with low $n$ and $B / T$ values should be produced by transformation of late-type galaxies within high-density environments or by passive evolution of late-type spirals.

The morphological classifications of large galaxy samples that have recently appeared in the literature will in the near future provide important clues to understanding the formation and evolution of galaxies, including lenticulars. These classifications provide a unique opportunity for selecting large samples of lenticular galaxies located in a large diversity of environments in a homogeneous way, from field to galaxy clusters. These large samples will permit us to solve the biases of present small galaxy samples. Detailed analysis of the structural parameters, together with the analysis of the environments where lenticulars live, will in the near future provide new and important constrains to the formation and evolution of lenticular galaxies.

\section{Acknowledgment}

The authors would like to thank the anonymous referees for helping him to improve both the content and the presentation of the paper. This work was supported by the projects AYA2010-21887-C04-04 and by the ConsoliderIngenio 2010 Program grant CSD2006-00070.

\section{References}

[1] A. Sandage, "The classification of galaxies: early history and ongoing developments," Annual Review of Astronomy and Astrophysics, vol. 43, pp. 581-624, 2005.

[2] R. Buta, "Galaxy morphology," Astrophysics, in press, http:// arxiv.org/abs/1102.0550.

[3] J. H. Reynolds, "Nebula, Photometric measures of the nuclei of some typical spiral nebul\&aelig," Monthly Notices of the Royal Astronomical Society, vol. 80, p. 746, 1920.

[4] E. P. Hubble, "Extragalactic nebulae," Astrophysical Journal, vol. 64, pp. 321-369, 1926.

[5] G. de Vaucouleurs, "Classification and morphology of external galaxies," Handbuch der Physik, vol. 53, p. 275, 1959.

[6] E. P. Hubble, Realm of the Nebulae, Yale University Press, New Haven, Conn, USA, 1936.

[7] A. Sandage, "The stellar and gaseous content of galaxies varies systematically along the morphological sequence from E to Sm. Variations along the spirals from Sa to Sd," in Galaxies and the Universe, A. Sandage, M. Sandage, and J. Kristian, Eds., vol. 9 of Stars and stellar Systems, p. 1, University of Chicago Press, Chicago, Ill, USA, 1975.

[8] A. Sandage and G. A. Tammann, A Revised Shapley-Ames Catalog of Bright Galaxies, Carnegie Institute of Washington, Washington, DC, USA, 1981.

[9] A. Sandage and J. Bedke, The Carnegie Atlas of Galaxies, Carnegie Institute of Washington, Washington, DC, USA, 1994.

[10] A. Sandage, The Hubble Atlas of Galaxies, Carnegie Institute of Washington, Washington, DC, USA, 1961.

[11] G. de Vaucouleurs, "Revised classification of 1500 bright galaxies," Astrophysical Journal Supplement, vol. 8, p. 31, 1963.
[12] G. de Vaucouleurs, A. de Vaucouleurs, H. G. Corwin Jr. et al., Third Reference Catalogue of Bright Galaxies, vol. 1-3, Springer, Berlin, Germany, 1991.

[13] S. van den Bergh, "What are S0 galaxies?" Astrophysical Journal, vol. 348, pp. 57-60, 1990.

[14] S. Van Den Bergh, "The nature of S0 galaxies," The Astronomical Journal, vol. 107, no. 1, pp. 153-159, 1994.

[15] S. Van Den Bergh, "What are S0(0) galaxies?" Astrophysical Journal, vol. 601, no. 1, pp. L37-L38, 2004.

[16] S. Van Den Bergh, "What are s0 galaxies?" Astrophysical Journal Letters, vol. 694, no. 2, pp. L120-L122, 2009.

[17] E. Emsellem, M. Cappellari, and D. Krajnovic, "The SAURON project-IX. A kinematic classification for early-type galaxies," Monthly Notices of the Royal Astronomical Society, vol. 379, no. 2, pp. 401-417, 2007.

[18] M. Cappellari, E. Emsellem, D. Krajnovic et al., "The ATLAS3D project-VII. A new look at the morphology of nearby galaxies: the kinematic morphology-density relation," Monthly Notices of the Royal Astronomical Society, vol. 416, no. 3, pp. 1680-1696, 2011.

[19] D. Krajnović, E. Emsellem, M. Cappellari et al., "The ATLAS3D project-II. Morphologies, kinemetric features and alignment between photometric and kinematic axes of early-type galaxies," Monthly Notices of the Royal Astronomical Society, vol. 414, no. 4, pp. 2923-2949, 2011.

[20] S. van den Bergh, "A new classification system for galaxies," Astrophysical Journal, vol. 206, no. 1, pp. 883-887, 1976.

[21] E. Laurikainen, H. Salo, R. Buta, J. H. Knapen, and S. Comeron, "Photometric scaling relations of lenticular and spiral galaxies," Monthly Notices of the Royal Astronomical Society, vol. 405, no. 2, pp. 1089-1118, 2010.

[22] J. Kormendy and R. Bender, "A revised parallel-sequence morphological classification of galaxies: structure and formation of S0 and spheroidal galaxies," The Astrophysical Journal Supplement, vol. 198, no. 1, article 2, 2012.

[23] M. Bernardi, F. Shankar, J. B. Hyde, S. Mei, F. Marulli, and R. K. Sheth, "Galaxy luminosities, stellar masses, sizes, velocity dispersions as a function of morphological type," Monthly Notices of the Royal Astronomical Society, vol. 404, no. 4, pp. 2087-2122, 2010.

[24] E. Laurikainen, H. Salo, R. Buta, and J. H. Knapen, "arinfrared atlas of S0-Sa galaxies (NIRS0S)," Monthly Notices of the Royal Astronomical Society, vol. 418, no. 3, pp. 1452-1490, 2011.

[25] K. Abazajian, J. K. Adelman-McCarthy, M. A. Agüeros et al., "The first data release of the sloan digital sky survey," The Astronomical Journal, vol. 126, no. 4, pp. 2081-2086, 2003.

[26] S. Folkes, S. Ronen, I. Price et al., "The $2 \mathrm{dF}$ galaxy redshift survey: spectral types and luminosity functions," Monthly Notices of the Royal Astronomical Society, vol. 308, no. 2, pp. 459-472, 1999.

[27] M. Fukugita, O. Nakamura, S. Okamura et al., "A catalog of morphologically classified galaxies from the sloan digital sky survey: north equatorial region," The Astronomical Journal, vol. 134, no. 2, pp. 579-593, 2007.

[28] P. B. Nair and R. G. Abraham, "A catalog of detailed visual morphological classifications for 14,034 galaxies in the sloan digital sky survey," Astrophysical Journal, Supplement Series, vol. 186, no. 2, pp. 427-456, 2010.

[29] C. J. Lintott, K. Schawinski, A. Slosar et al., "Galaxy Zoo: morphologies derived from visual inspection of galaxies from the Sloan Digital Sky Survey," Monthly Notices of the Royal Astronomical Society, vol. 389, no. 3, pp. 1179-1189, 2008. 
[30] C. Lintott, K. Schawinski, S. Bamford et al., "Galaxy Zoo 1: data release of morphological classifications for nearly 900000 galaxies," Monthly Notices of the Royal Astronomical Society, vol. 410, no. 1, pp. 166-178, 2011.

[31] G. de Vaucouleurs, "Integrated colors of bright galaxies in the u, b, V system," Astrophysical Journal Supplement, vol. 5, p. 233, 1961.

[32] I. Strateva, Ž. Ivezić, G. R. Knapp et al., "Color separation of galaxy types in the Sloan Digital Sky Survey imaging data," The Astronomical Journal, vol. 122, no. 4, pp. 1861-1874, 2001.

[33] M. L. Humason, "Apparent velocity-shifts in the spectra of faint nebulae," Astrophysical Journal, vol. 74, p. 35, 1931.

[34] W. W. Morgan and N. U. Mayall, "A spectral classification of galaxies," Publications of the Astronomical Society of the Pacific, vol. 69, no. 409, p. 291, 1957.

[35] J. A. Baldwin, M. M. Phillips, and R. Terlevich, "Classification parameters for the emission-line spectra of extragalactic objects," Publications of the Astronomical Society of the Pacific, vol. 93, pp. 5-19, 1981.

[36] D. E. Osterbrock and S. Veilleux, "Spectral classification of emission-line galaxies," Publications of the Astronomical Society of the Pacific, vol. 98, p. 1106, 1986.

[37] S. Veilleux and D. E. Osterbrock, "Spectral classification of emission-line galaxies," Astrophysical Journal Supplement, vol. 63, pp. 295-310, 1987.

[38] L. Sodré Jr. and H. Cuevas, "Spectral classification of galaxies," Vistas in Astronomy, vol. 38, no. 3, pp. 287-291, 1994.

[39] A. J. Connolly, A. S. Szalay, M. A. Bershady, A. L. Kinney, and D. Calzetti, "Spectral classification of galaxies: an orthogonal approach," The Astronomical Journal, vol. 110, no. 3, pp. 1071-1082, 1995.

[40] S. R. Folkes, O. Lahav, and S. J. Maddox, "An artificial neural network approach to the classification of galaxy spectra," Monthly Notices of the Royal Astronomical Society, vol. 283, no. 2, pp. 651-665, 1996.

[41] D. Zaritsky, A. I. Zabludoff, and J. A. Willick, "Spectral classification of galaxies along the hubble sequence," The Astronomical Journal, vol. 110, no. 4, pp. 1602-1613, 1995.

[42] B. C. Bromley, W. H. Press, H. Lin, and R. P. Kirshner, "Spectral classification and luminosity function of galaxies in the las campanas redshift survey," Astrophysical Journal Letters, vol. 505, no. 1, pp. 25-36, 1998.

[43] J. S. Almeida, J. A. L. Aguerri, C. Muñoz-Tuñón, and A. De Vicente, "Automatic unsupervised classification of all sloan digital sky survey data release 7 galaxy spectra," Astrophysical Journal Letters, vol. 714, no. 1, pp. 487-504, 2010.

[44] J. Sánchez Almeida, J. A. L. Aguerri, C. Muñoz-Tuñón, and M. Huertas-Company, "Relationship between Hubble type and spectroscopic class in local galaxies," Astrophysical Journal Letters, vol. 735, no. 2, article 125, 2011.

[45] W. W. Morgan, "A preliminary classification of the forms of galaxies according to their stellar population," Publications of the Astronomical Society of the Pacific, vol. 70, no. 415, p. 364, 1958.

[46] W. W. Morgan, "Preliminary classification of the forms of galaxies according to their stellar population. II," Publications of the Astronomical Society of the Pacific, vol. 71, no. 422, p. 394, 1959.

[47] C. W. Fraser, "Concentration indices of galaxies," The Observatory, vol. 92, pp. 51-54, 1972.

[48] G. de Vaucouleurs, "Contributions to the galaxy photometry. I-standard total magnitudes, luminosity curves, and photometric parameters of 115 bright galaxies in the B system from detailed surface photometry," Astrophysical Journal Supplement, vol. 33, pp. 211-218, 1977.

[49] S. Okamura, K. Kodaira, and M. Watanabe, "Digital surface photometry of galaxies toward a quantitative classification. III-a mean concentration index as a parameter representing the luminosity distribution," Astrophysical Journal, vol. 280, pp. 7-14, 1984.

[50] G. Gavazzi, B. Garilli, and A. Boselli, "A CCD survey of galaxies in the Coma supercluster," Astronomy and Astrophysics Supplement, vol. 83, no. 3, pp. 399-458, 1990.

[51] R. G. Abraham, F. Valdes, H. K. C. Yee, and S. Van Den Bergh, "The morphologies of distant galaxies. I. An automated classification system," Astrophysical Journal Letters, vol. 432, no. 1, pp. 75-90, 1994.

[52] R. G. Abraham, S. Van Den Bergh, K. Glazebrook et al., “The morphologies of distant galaxies. II. Classifications from the hubble space telescope medium deep survey," Astrophysical Journal, Supplement Series, vol. 107, no. 1, pp. 1-17, 1996.

[53] C. J. Conselice, M. A. Bershady, and A. Jangren, "The asymmetry of galaxies: physical morphology for nearby and high-redshift galaxies," Astrophysical Journal, vol. 529, no. 2, pp. 886-910, 2000.

[54] R. G. Abraham, S. Van den Bergh, and P. Nair, "A new approach to galaxy morphology. I. Analysis of the Sloan Digital Sky Survey early data release," Astrophysical Journal Letters, vol. 588, no. 1, pp. 218-229, 2003.

[55] J. M. Lotz, J. Primack, and P. Madau, "A new nonparametric approach to galaxy morphological classification," The Astronomical Journal, vol. 128, no. 1, pp. 163-182, 2004.

[56] C. Scarlata, C. M. Carollo, S. Lilly et al., "Cosmos morphological classification with the Zurich estimator of structural types (ZEST) and the evolution since $\mathrm{z}=1$ of the luminosity function of early, disk, and irregular galaxies," Astrophysical Journal, Supplement Series, vol. 172, no. 1, pp. 406-433, 2007.

[57] M. A. Bershady, A. Jangren, and C. J. Conselice, "Structural and photometric classification of galaxies. I. Calibration based on a nearby galaxy sample," The Astronomical Journal, vol. 119, no. 6, pp. 2645-2663, 2000.

[58] M. Huertas-Company, D. Rouan, L. Tasca, G. Soucail, and O. Le Fèvre, "A robust morphological classification of highredshift galaxies using support vector machines on seeing limited images: I. Method description," Astronomy and Astrophysics, vol. 478, no. 3, pp. 971-980, 2008.

[59] M. Huertas-Company, D. Rouan, G. Soucail, O. Le Fèvre, L. Tasca, and T. Contini, "Morphological evolution of $\mathrm{z} \sim$ 1 galaxies from deep $\mathrm{K}$-band $\mathrm{AO}$ imaging in the COSMOS deep field," Astronomy and Astrophysics, vol. 468, no. 3, pp. 937-950, 2007.

[60] M. Huertas-Company, L. Tasca, D. Rouan et al., "A robust morphological classification of high-redshift galaxies using support vector machines on seeing limited images," Astronomy and Astrophysics, vol. 497, no. 3, pp. 743-753, 2009.

[61] M. Huertas-Company, J. A. L. Aguerri, L. Tresse, M. Bolzonella, A. M. Koekemoer, and C. Maier, "Evolution of blue $\mathrm{E} / \mathrm{S} 0$ galaxies from $\mathrm{z} \sim 1$ : merger remnants or disk-rebuilding galaxies?" Astronomy and Astrophysics, vol. 515, no. 1, article A3, 2010 .

[62] M. Huertas-Company, J. A. L. Aguerri, M. Bernardi, S. Mei, and J. Sánchez Almeida, "Revisiting the Hubble sequence in the SDSS DR7 spectroscopic sample: a publicly available Bayesian automated classification," Astronomy and Astrophysics, vol. 525, no. 10, article A157, 2011.

[63] J. A. L. Aguerri, N. Elias-Rosa, E. M. Corsini, and C. MuñozTuñón, "Photometric properties and origin of bulges in SB0 
galaxies," Astronomy and Astrophysics, vol. 434, no. 1, pp. 109-122, 2005.

[64] M. Prieto, J. A. L. Aguerri, A. M. Varela, and C. MuñozTuñón, "Optical surface photometry of a sample of disk galaxies. II. Structural components," Astronomy and Astrophysics, vol. 367, no. 2, pp. 405-427, 2001.

[65] E. Laurikainen, H. Salo, R. Buta, and J. H. Knapen, "Properties of bars and bulges in the Hubble sequence," Monthly Notices of the Royal Astronomical Society, vol. 381, no. 1, pp. 401-417, 2007.

[66] E. Laurikainen, H. Salo, R. Buta, and J. H. Knapen, "Bars, ovals, and lenses in early-type disk galaxies: probes of galaxy evolution," Astrophysical Journal Letters, vol. 692, no. 1, pp. L34-L39, 2009.

[67] M. A. Shaw and G. Gilmore, "The luminosity distributions of edge-on spiral galaxies. I- a two-dimensional model and its application to NGC 891 and 4565," Monthly Notices of the Royal Astronomical Society, vol. 237, pp. 903-927, 1989.

[68] Y. I. Byun and K. C. Freeman, "Two-dimensional decomposition of bulge and disk," Astrophysical Journal Letters, vol. 448, no. 2, pp. 563-574, 1995.

[69] R. S. de Jong, "Near-infrared and optical broadband surface photometry of 86 face-on disk dominated galaxies. II. A two-dimensional method to determine bulge and disk parameters," Astronomy and Astrophysics Supplement, vol. 118, pp. 557-573, 1996.

[70] E. Laurikainen, H. Salo, and R. Buta, "Multicomponent decompositions for a sample of S0 galaxies," Monthly Notices of the Royal Astronomical Society, vol. 362, no. 4, pp. 13191347, 2005.

[71] C. Y. Peng, L. C. Ho, C. D. Impey, and H. W. Rix, "Detailed structural decomposition of galaxy images," The Astronomical Journal, vol. 124, no. 1, pp. 266-293, 2002.

[72] C. Y. Peng, L. C. Ho, C. D. Impey, and H. W. Rix, "Detailed decomposition of galaxy images. II. beyond axisymmetric models," The Astronomical Journal, vol. 139, no. 6, pp. 2097 2129, 2010.

[73] T. Weinzirl, S. Jogee, S. Khochfar, A. Burkert, and J. Kormendy, "Bulge $\mathrm{n}$ and $\mathrm{B} / \mathrm{T}$ in high-mass galaxies: constraints on the origin of bulges in hierarchical models," Astrophysical Journal Letters, vol. 696, no. 1, pp. 411-447, 2009.

[74] J. H. Reynolds, "The light curve of the Andromeda nebula (NGC 224)," Monthly Notices of the Royal Astronomical Society, vol. 74, pp. 132-136, 1913.

[75] E. P. Hubble, "Distribution of luminosity in elliptical nebulae," Astrophysical Journal, vol. 71, pp. 231-276, 1930.

[76] I. King, "The structure of star clusters. I. An empirical density law," The Astronomical Journal, vol. 67, p. 471, 1962.

[77] H. J. Rood, T. L. Page, E. C. Kintner, and I. R. King, "The structure of the coma cluster of galaxies," Astrophysical Journal, vol. 175, p. 627, 1972.

[78] A. Oemler Jr., "The structure of elliptical and cD galaxies," Astrophysical Journal, vol. 209, pp. 693-709, 1976.

[79] A. W. Graham, "A review of elliptical and disc galaxy structure, and modern scaling laws," in press, http://arxiv.org/ abs/1108.0997.

[80] G. de Vaucouleurs, "Recherches sur les Nebuleuses Extragalactiques," Annales d'Astrophysique, vol. 11, p. 247, 1948.

[81] G. de Vaucouleurs, "On the distribution of mass and luminosity in elliptical galaxies," Monthly Notices of the Royal Astronomical Society, vol. 113, p. 134, 1953.

[82] G. de Vaucouleurs, "Photoelectric photometry of the Andromeda nebula in the UBV system," Astrophysical Journal, vol. 128 , p. $465,1958$.
[83] J. Kormendy, "Brightness distributions in compact and normal galaxies. II-structure parameters of the spheroidal component," Astrophysical Journal, vol. 218, pp. 333-346, 1977.

[84] J. Kormendy, "Brightness distributions in compact and normal galaxies. III-decomposition of observed profiles into spheroid and disk components," Astrophysical Journal, vol. 217, pp. 406-419, 1977.

[85] S. M. Kent, "CCD surface photometry of field Galaxies. IIbulge/disk decompositions," Astrophysical Journal Supplement, vol. 59, pp. 115-159, 1985.

[86] F. Simien and G. de Vaucouleurs, "Systematics of bulgeto-disk ratios," Astrophysical Journal, vol. 302, pp. 564-578, 1986.

[87] J. Kormendy and A. G. Bruzual, "The minor-axis brightness profile of the spiral galaxy NGC 4565 and the problem of massive halos," Astrophysical Journal, vol. 223, pp. L63-L66, 1978.

[88] D. Burstein, "Structure and origin of S0 galaxies. II-diskto-bulge ratios," Astrophysical Journal, vol. 234, pp. 435-447, 1979.

[89] R. J. Wainscoat, K. C. Freeman, and A. R. Hyland, "The optical and near-infrared distribution of light in the edge-on galaxy IC 2531," Astrophysical Journal, vol. 337, pp. 163-190, 1989.

[90] S. M. Kent, T. M. Dame, and G. Fazio, "Galactic structure from the Spacelab infrared telescope. II. Luminosity models of the milky way," Astrophysical Journal Letters, vol. 378, no. 1, pp. 131-138, 1991.

[91] Y. C. Andredakis and R. H. Sanders, "Exponential bulges in late-type spirals: an improved description of the light distribution," Monthly Notices of the Royal Astronomical Society, vol. 267, no. 2, pp. 283-296, 1994.

[92] J. L. Sérsic, Atlas de Galaxias Australes, Observatorio Astronomico, Cordoba, Argentina, 1968.

[93] Y. C. Andredakis, R. F. Peletier, and M. Balcells, "The shape of the luminosity profiles of bulges of spiral Galaxies," Monthly Notices of the Royal Astronomical Society, vol. 275, no. 3, p. 874, 1995.

[94] R. S. de Jong, "Near-infrared and optical broadband surface photometry of 86 face-on disk dominated galaxies. III. The statistics of the disk and bulge parameters," Astronomy and Astrophysics, vol. 313, pp. 45-64, 1996.

[95] S. Courteau, R. S. de Jong, and A. H. Broeils, "Evidence for secular evolution in late-type spirals," Astrophysical Journal Letters, vol. 457, no. 2, pp. L73-L76, 1996.

[96] A. W. Graham, "An investigation into the prominence of spiral galaxy bulges," The Astronomical Journal, vol. 121, no. 2, pp. 820-840, 2001.

[97] C. Möllenhoff and J. Heidt, "Surface photometry of spiral galaxies in NIR: structural parameters of disks and bulges," Astronomy and Astrophysics, vol. 368, no. 1, pp. 16-37, 2001.

[98] I. Trujillo, J. A. L. Aguerri, C. M. Gutiérrez, and J. Cepa, "Quantitative morphology of the intermediate-redshift galaxy Cluster Abell 2443 from ground-based imaging: evidence for a galaxy concentration index correlation with cluster density," The Astronomical Journal, vol. 122, no. 1, pp. 38-54, 2001.

[99] N. Castro-Rodríguez and F. Garzón, "NIR surface photometry of a sample of nearby spiral galaxies," Astronomy and Astrophysics, vol. 411, pp. 55-62, 2003.

[100] C. M. Gutiérrez, I. Trujillo, J. A. L. Aguerri, A. W. Graham, and N. Caon, "Quantitative morphology of galaxies in the 
core of the Coma Cluster," Astrophysical Journal, vol. 602, no. 2, pp. 664-677, 2004.

[101] J. A. L. Aguerri, J. Iglesias-Paramo, J. M. Vilchez, and C. Muñoz-Tuñön, "Environmental effects in the structural parameters of galaxies in the Coma cluster," The Astronomical Journal, vol. 127, no. 3, pp. 1344-1359, 2004.

[102] J. A. L. Aguerri, J. Iglesias-PÁRAMO, J. M. Vílchez, C. Muñoz-Tuñón, and R. Sánchez-Janssen, "Structural parameters of dwarf galaxies in the Coma Cluster: on the origin of dS0 galaxies," The Astronomical Journal, vol. 130, no. 2, pp. 475-495, 2005.

[103] J. Méndez-Abreu, J. A. L. Aguerri, E. M. Corsini, and E. Simonneau, "Structural properties of disk galaxies I. The intrinsic equatorial ellipticity of bulges," Astronomy and Astrophysics, vol. 478, no. 2, pp. 353-369, 2008.

[104] J. Méndez-Abreu, E. Simonneau, J. A. L. Aguerri, and E. M. Corsini, "Structural properties of disk galaxies: II. Intrinsic shape of bulges," Astronomy and Astrophysics, vol. 521, no. 7, article A71, 2010.

[105] P. D. Allen, S. P. Driver, A. W. Graham et al., "The Millennium Galaxy Catalogue: bulge-disc decomposition of 10095 nearby galaxies," Monthly Notices of the Royal Astronomical Society, vol. 371, no. 1, pp. 2-18, 2006.

[106] M. Capaccioli, "Photometry of early-type galaxies and the $\mathrm{R}$ exp 1/4 law," in The World of Galaxies, H. G. Corwin and L. Bottinelli, Eds., Le Monde des Galaxies, pp. 208-227, Springer, Berlin, Germany, 1989.

[107] L. Ciotti, "Stellar systems following the $\mathrm{R}^{1 / m}$ luminosity law," Astronomy and Astrophysics, vol. 249, no. 1, pp. 99-106, 1991.

[108] L. Ciotti and G. Bertin, "Analytical properties of the $\mathrm{R}^{1 / m}$ law," Astronomy and Astrophysics, vol. 352, no. 2, pp. 447451, 1999.

[109] E. Simonneau and F. Prada, "Structural properties of spherical galaxies: a semi-analytical approach," Revista Mexicana de Astronomía y Astrofísica, vol. 40, pp. 69-79, 2004.

[110] A. W. Graham and S. P. Driver, "A concise reference to (projected) sérsic $\mathrm{R}^{1 / n}$ quantities, including concentration, profile slopes, petrosian indices, and kron magnitudes," Publications of the Astronomical Society of Australia, vol. 22, no. 2, pp. 118127, 2005.

[111] K. C. Freeman, "On the disks of spiral and so galaxies," Astrophysical Journal, vol. 160, p. 811, 1970.

[112] M. Pohlen and I. Trujillo, "The structure of galactic disks: studying late-type spiral galaxies using SDSS," Astronomy and Astrophysics, vol. 454, no. 3, pp. 759-772, 2006.

[113] B. G. Elmegreen and D. M. Elmegreen, "Properties of barred spiral galaxies," Astrophysical Journal, vol. 288, pp. 438-455, 1985.

[114] D. Alexei Gadotti, "Image decomposition of barred galaxies and AGN hosts," Monthly Notices of the Royal Astronomical Society, vol. 384, no. 1, pp. 420-439, 2008.

[115] J. A. L. Aguerri, V. P. Debattista, and E. M. Corsini, "Measurement of fast bars in a sample of early-type barred galaxies," Monthly Notices of the Royal Astronomical Society, vol. 338, no. 2, pp. 465-480, 2003.

[116] M. Prieto, S. T. Gottesman, J. A. L. Aguerri, and A. M. Varela, "Structural components of NGC 5850," The Astronomical Journal, vol. 114, no. 4, pp. 1413-1426, 1997.

[117] K. C. Freeman, "Structure and evolution of barred spiral galaxies, I," Monthly Notices of the Royal Astronomical Society, vol. 133, pp. 47-62, 1966.
[118] J. A. L. Aguerri, J. Méndez-Abreu, and E. M. Corsini, “The population of barred galaxies in the local universe: I. Detection and characterisation of bars," Astronomy and Astrophysics, vol. 495, no. 2, pp. 491-504, 2009.

[119] J. Kormendy, "A morphological survey of bar, lens, and ring components in galaxies Secular evolution in galaxy structure," Astrophysical Journal, vol. 227, pp. 714-728, 1979.

[120] M. F. Duval and E. Athanassoula, "Photometry, kinematics, and dynamics of the barred spiral galaxy NGC 5383," Astronomy and Astrophysics, vol. 121, no. 2, pp. 297-312, 1983.

[121] S. M. Kent, "Dark matter in spiral galaxies. I-galaxies with optical rotation curves," The Astronomical Journal, vol. 91, pp. 1301-1327, 1986.

[122] B. Binggeli and L. M. Cameron, "Dwarf galaxies in the Virgo cluster. I-the systematic photometric properties of earlytype dwarfs," Astronomy and Astrophysics, vol. 252, no. 1, pp. 27-52, 1991.

[123] N. Caon, M. Capaccioli, and M. D’Onofrio, "On the shape of the light profiles of early type galaxies," Monthly Notices of the Royal Astronomical Society, vol. 265, no. 4, p. 1013, 1993.

[124] A. W. Graham and R. Guzmán, "HST photometry of dwarf elliptical galaxies in coma, and an explanation for the alleged structural dichotomy between dwarf and bright elliptical galaxies," The Astronomical Journal, vol. 125, no. 6, pp. 29362950, 2003.

[125] H. G. Khosroshahi, Y. Wadadekar, and A. Kembhavi, "Correlations among global photometric properties of disk galaxies," Astrophysical Journal, vol. 533, no. 1, pp. 162-171, 2000.

[126] L. A. MacArthur, S. Corteau, and J. A. Holtzman, "Structure of disk-dominated galaxies. I. Bulge/disk parameters, simulations, and secular evolution," Astrophysical Journal Letters, vol. 582, no. 2, pp. 689-722, 2003.

[127] I. Trujillo and J. A. L. Aguerri, "Quantitative morphological analysis of the Hubble Deep Field North and Hubble Deep Field South-I. Early- and late-type luminosity-size relations of galaxies out to $\mathrm{z} \sim 1$," Monthly Notices of the Royal Astronomical Society, vol. 355, no. 1, pp. 82-96, 2004.

[128] C. Mollenhoff, "Disk-bulge decompositions of spiral galaxies in UBVRI," Astronomy and Astrophysics, vol. 415, no. 1, pp. 63-76, 2004.

[129] L. K. Hunt, D. Pierini, and C. Giovanardi, "Near-infrared observations of galaxies in pisces-perseus. V. On the origin of bulges," Astronomy and Astrophysics, vol. 414, no. 3, pp. 905-918, 2004.

[130] M. Balcells, A. W. Graham, and R. F. Peletier, "Galactic bulges from hubble space telescope NICMOS observations: global scaling relations," Astrophysical Journal Letters, vol. 665, no. 2, pp. 1104-1114, 2007.

[131] D. B. Fisher and N. Drory, "The structure of classical bulges and pseudobulges: the link between pseudobulges and SRIC index," The Astronomical Journal, vol. 136, no. 2, pp. 773-839, 2008.

[132] A. Dressler, D. Lynden-Bell, and D. Burstein, "Spectroscopy and photometry of elliptical galaxies. I-a new distance estimator," Astrophysical Journal, vol. 313, pp. 42-58, 1987.

[133] S. Djorgovski and M. Davis, "Fundamental properties of elliptical galaxies," Astrophysical Journal, vol. 313, pp. 59-68, 1987.

[134] J. Falcón-Barroso, R. F. Peletier, and M. Balcells, "Bulges on the Fundamental Plane of early-type galaxies," Monthly Notice of the Royal Astronomical Society, vol. 335, no. 3, pp. 741-752, 2002.

[135] C. D. Ravikumar, S. Barway, A. Kembhavi, B. Mobasher, and V. C. Kuriakose, "Photometric scaling relations for bulges 
of galaxies," Astronomy and Astrophysics, vol. 446, no. 3, pp. 827-838, 2006.

[136] S. Barway, Y. Wadadekar, A. K. Kembhavi, and Y. D. Mayya, "Near-infrared bulge-disc correlations of lenticular galaxies," Monthly Notices of the Royal Astronomical Society, vol. 394, no. 4, pp. 1991-2000, 2009.

[137] A. Nigoche-Netro, J. A. L. Aguerri, P. Lagos, A. RuelasMayorga, L. J. Sánchez, and A. MacHado, "The FaberJackson relation for early-type galaxies: dependence on the magnitude range," Astronomy and Astrophysics, vol. 516, no. 16, article A96, 2010.

[138] A. Matković and R. Guzmán, "Kinematic properties and stellar populations of faint early-type galaxies-I. Velocity dispersion measurements of central Coma galaxies," Monthly Notices of the Royal Astronomical Society, vol. 362, no. 1, pp. 289-300, 2005.

[139] E. Noordermeer and J. M. Van Der Hulst, "The stellar mass distribution in early-type disc galaxies: surface photometry and bulge-disc decompositions," Monthly Notices of the Royal Astronomical Society, vol. 376, no. 4, pp. 1480-1512, 2007.

[140] A. W. Graham and C. C. Worley, "Inclination- and dustcorrected galaxy parameters: bulge-to-disc ratios and sizeluminosity relations," Monthly Notices of the Royal Astronomical Society, vol. 388, no. 4, pp. 1708-1728, 2008.

[141] J. Kormendy and R. C. Kennicutt Jr., "Secular evolution and the formation of pseudobulges in disk galaxies," Annual Review of Astronomy and Astrophysics, vol. 42, pp. 603-683, 2004.

[142] D. A. Gadotti, "Structural properties of pseudo-bulges, classical bulges and elliptical galaxies: a Sloan Digital Sky Survey perspective," Monthly Notices of the Royal Astronomical Society, vol. 393, no. 4, pp. 1531-1552, 2009.

[143] D. B. Fisher and N. Drory, "Bulges of nearby galaxies with Spitzer: scaling relations in pseudobulges and classical bulges," Astrophysical Journal Letters, vol. 716, no. 2, pp. 942969, 2010.

[144] D. Zaritsky and K. Y. Lo, "Evidence for nonaxisymmetric nuclear bulges in spiral galaxies," Astrophysical Journal, vol. 303, pp. 66-75, 1986.

[145] F. Bertola, M. Vietri, and W. W. Zeilinger, "Triaxiality in disk galaxies," Astrophysical Journal Letters, vol. 374, no. 1, pp. L13-L16, 1991.

[146] J. E. Beckman, A. M. Varela, C. Munoz-Tunon, J. M. Vilchez, and J. Cepa, "Observational evidence for triaxiality, and for relatively recent star formation in the bulge of NGC 4736," Astronomy and Astrophysics, vol. 245, no. 2, pp. 436-448, 1991.

[147] A. M. Varela, C. Munoz-Tunon, and E. Simmoneau, "Spatial source distribution of bulges from surface photometry: application to NGC 2841," Astronomy and Astrophysics, vol. 306, no. 2, pp. 381-390, 1996.

[148] E. Simonneau, A. M. Varela, and C. Munoz-Tunon, "Astronomical observations and synthesis of ellipsoidal triaxial structures. I. Geometrical considerations," Nuovo Cimento B, vol. 113, no. 7, pp. 927-944, 1998.

[149] B. Lindblad, "On the evolution of stellar systems," Vistas in Astronomy, vol. 2, pp. 1711-1721, 1956.

[150] O. E. Gerhard and M. Vietri, "The peculiar shape of the inner galactic rotation curve," Monthly Notices of the Royal Astronomical Society, vol. 223, no. 2, p. 377, 1986.

[151] F. Bertola, W. W. Zeilinger, and V. C. Rubin, "Evidence for a triaxial bulge in the spiral galaxy NGC 4845," Astrophysical Journal, vol. 345, pp. L29-L32, 1989.
[152] O. E. Gerhard, M. Vietri, and S. M. Kent, "The triaxial bulge of the SA galaxy NGC 4845," Astrophysical Journal, vol. 345, pp. L33-L36, 1989.

[153] S. Berman, "Hydrodynamic simulations of the triaxial bulge of M 31," Astronomy and Astrophysics, vol. 371, no. 2, pp. 476-486, 2001.

[154] E. M. Corsini, A. Pizzella, L. Coccato, and F. Bertola, "Minoraxis velocity gradients in spirals and the case of inner polar disks," Astronomy and Astrophysics, vol. 408, no. 3, pp. 873885, 2003.

[155] L. Coccato, E. M. Corsini, A. Pizzella, L. Morelli, J. G. S J Funes, and F. Bertola, "Minor-axis velocity gradients in disk galaxies," Astronomy and Astrophysics, vol. 416, no. 2, pp. 507-514, 2004.

[156] L. Coccato, E. M. Corsini, A. Pizzella, and F. Bertola, "Spiral galaxies with a central plateau in the gas velocity curve along the major axis," Astronomy and Astrophysics, vol. 440, no. 1, pp. 107-109, 2005.

[157] K. Fathi and R. F. Peletier, "Do bulges of early- and late-type spirals have different morphology?" Astronomy and Astrophysics, vol. 407, pp. 61-74, 2003.

[158] M. Franx, G. Illingworth, and T. Heckman, "Major and minor axis kinematics of 22 ellipticals," Astrophysical Journal, vol. 344, pp. 613-636, 1989.

[159] R. B. Tully and J. R. Fisher, "A new method of determining distances to galaxies," Astronomy and Astrophysics, vol. 54, no. 3, pp. 661-673, 1977.

[160] V. C. Rubin, D. Burstein, W. K. Ford Jr., and N. Thonnard, "Rotation velocities of 16 SA galaxies and a comparison of $\mathrm{Sa}, \mathrm{Sb}$, and SC rotation properties," Astrophysical Journal, vol. 289, pp. 81-98, 1985.

[161] R. Giovanelli, M. P. Haynes, T. Herter et al., "The I band Tully-Fisher relation for cluster galaxies: a template relation. Its scatter and bias corrections," The Astronomical Journal, vol. 113, no. 1, pp. 53-79, 1997.

[162] J. L. Hinz, G. H. Rieke, and N. Caldwell, "The TullyFisher relation in Coma and Virgo Cluster s0 galaxies," The Astronomical Journal, vol. 126, no. 6, pp. 2622-2634, 2003.

[163] E. Neistein, D. Maoz, H. W. Rix, and J. L. Tonry, "A tullyfisher relation for S0 galaxies," The Astronomical Journal, vol. 117, no. 6, pp. 2666-2675, 1999.

[164] J. L. Hinz, R. Hans-Walter, and G. M. Bernstein, "A comparison of Coma Cluster S0 galaxies with the Tully-Fisher relation for late-type spirals," The Astronomical Journal, vol. 121, no. 2, pp. 683-691, 2001.

[165] A. Mathieu, M. R. Merrifield, and K. Kuijken, "The dynamics of S0 galaxies and their Tully-Fisher relation," Monthly Notices of the Royal Astronomical Society, vol. 330, no. 2, pp. 251258, 2002.

[166] A. G. Bedregal, A. Aragon-Salamanca, and M. R. Merrifield, "The Tully-Fisher relation for S0 galaxies," Monthly Notices of the Royal Astronomical Society, vol. 373, no. 3, pp. 1125-1140, 2006.

[167] M. J. Williams, M. Bureau, and M. Cappellari, "The TullyFisher relations of early-type spiral and S0 galaxies," Monthly Notices of the Royal Astronomical Society, vol. 409, no. 4, pp. 1330-1346, 2010.

[168] M. Prieto, A. C. Aguilar, and C. García, "V-R diagram for spiral galaxies. New insights into the hubble sequence," Astrophysics and Space Science, vol. 157, no. 1-2, pp. 321-329, 1989.

[169] A. Campos-Aguilar, M. Prieto, and C. Garcia, "The V-R diagram-a diagnostic tool for the dynamical classification of spiral galaxies," Astronomy and Astrophysics, vol. 276, no. 1, p. 16, 1993. 
[170] S. Barway, A. Kembhavi, Y. Wadadekar, C. D. Ravikumar, and Y. D. Mayya, "Lenticular galaxy formation: possible luminosity dependence," Astrophysical Journal, vol. 661, no. 1, pp. L37-L40, 2007.

[171] K. L. Masters, R. C. Nichol, B. Hoyle et al., "Galaxy Zoo: bars in disc galaxies," Monthly Notices of the Royal Astronomical Society, vol. 411, no. 3, pp. 2026-2034, 2011.

[172] J. Méndez-Abreu, R. Sánchez-Janssen, and J. A. L. Aguerri, "Which galaxies host bars and disks? A study of the Coma cluster," The Astrophysical Journal Letters, vol. 711, pp. L61L65, 2010.

[173] I. Marinova and S. Jogee, "Characterizing bars at $\mathrm{z} \sim 0$ in the optical and NIR: implications for the evolution of barred disks with redshift," Astrophysical Journal, vol. 659, no. 2, pp. 1176-1197, 2007.

[174] F. D. Barazza, S. Jogee, and I. Marinova, "Bars in diskdominated and bulge-dominated galaxies at $\mathrm{z} \times 0$ : new insights from $~ 3600$ SDSS galaxies," Astrophysical Journal Letters, vol. 675, no. 2, pp. 1194-1212, 2008.

[175] H. Wozniak, D. Friedli, L. Martinet, P. Martin, and P. Bratschi, "Disc galaxies with multiple triaxial structures. I. BVRI and $\mathrm{H} \alpha$ surface photometry," Astronomy and Astrophysics Supplement, vol. 111, p. 115, 1995.

[176] S. Laine, I. Shlosman, J. H. Knapen, and R. F. Peletier, "Nested and single bars in Seyfert and non-Seyfert galaxies," Astrophysical Journal Letters, vol. 567, no. 1, pp. 97-117, 2002.

[177] K. Ohta, M. Hamabe, and K. I. Wakamatsu, "Surface photometry of barred spiral galaxies," Astrophysical Journal Letters, vol. 357, no. 1, pp. 71-90, 1990.

[178] J. A. L. Aguerri, J. E. Beckman, and M. Prieto, "Bar strengths, bar lengths, and corotation radii, derived photometrically for 10 barred galaxies," The Astronomical Journal, vol. 116, no. 5, pp. 2136-2153, 1998.

[179] J. A. L. Aguerri, C. Muñoz-Tuñón, A. M. Varela, and M. Prieto, "Characterizing bar structures: application to NGC 1300, NGC 7479 and NGC 7723," Astronomy and Astrophysics, vol. 361, no. 3, pp. 841-849, 2000.

[180] D. A. Gadotti, "Secular evolution and structural properties of stellar bars in galaxies," Monthly Notices of the Royal Astronomical Society, vol. 415, no. 4, pp. 3308-3318, 2011.

[181] S. Barway, Y. Wadadekar, and A. K. Kembhavi, "Bar fraction in lenticular galaxies: dependence on luminosity and environment," Monthly Notices of the Royal Astronomical Society, vol. 410, no. 1, pp. L18-L22, 2011.

[182] L. Giordano, K.-V.H. Tran, B. Moore, and A. Saintonge, "Multi-wavelength properties of barred galaxies in the local universe: environment and evolution across the hubble sequence," in press, http://arxiv.org/abs/1111.1532.

[183] I. Marinova, S. Jogee, T. Weinzirl et al., "The HST/ACS Coma cluster survey. VIII. barred disk galaxies in the core of the Coma Cluster," The Astrophysical Journal, vol. 746, no. 2, article 136, 2012.

[184] P. Martin, "Quantitative morphology of bars in spiral galaxies," The Astronomical Journal, vol. 109, no. 6, pp. 2428 2443, 1995.

[185] K. Sheth, M. W. Regan, N. Z. Scoville, and L. E. Strubbe, "Barred galaxies at $\mathrm{z}>0.7$ : NICMOS Hubble Deep FieldNorth observations," Astrophysical Journal Letters, vol. 592, no. 1, pp. L13-L16, 2003.

[186] P. Erwin, "How large are the bars in barred galaxies?" Monthly Notices of the Royal Astronomical Society, vol. 364, no. 1, pp. 283-302, 2005.
[187] A. C. Ouillen, J. A. Frogel, and R. A. Gonzalez, "The gravitational potential of the bar in NGC 4314," Astrophysical Journal Letters, vol. 437, no. 1, pp. 162-172, 1994.

[188] B. Hoyle, K. L. Masters, R. C. Nichol et al., "Galaxy Zoo: bar lengths in local disc galaxies," Monthly Notices of the Royal Astronomical Society, vol. 415, pp. 3627-3640, 2011.

[189] K. Menéndez-Delmestre, K. Sheth, E. Schinnerer, T. H. Jarrett, and N. Z. Scoville, "A near-infrared study of 2MASS bars in local galaxies: an anchor for high-redshift studies," Astrophysical Journal, vol. 657, no. 2, pp. 790-804, 2007.

[190] R. Buta, E. Laurikainen, H. Salo, and J. H. Knapen, "Decreased frequency of strong bars in S0 galaxies: evidence for secular evolution," Astrophysical Journal Letters, vol. 721, no. 1, pp. 259-266, 2010.

[191] F. Combes and R. H. Sanders, "Formation and properties of persisting stellar bars," Astronomy and Astrophysics, vol. 96, no. 1-2, pp. 164-173, 1981.

[192] R. Buta and D. L. Block, "A dust-penetrated classification scheme for bars as inferred from their gravitational force fields," Astrophysical Journal Letters, vol. 550, no. 1, pp. 243252, 2001.

[193] H. Salo, P. Rautiainen, R. Buta et al., "The structure and dynamics of the early-type resonance ring galaxy IC 4214. II. Models," The Astronomical Journal, vol. 117, no. 2, pp. 792810, 1999.

[194] H. Salo, E. Laurikainen, R. Buta, and J. H. Knapen, "Bars do drive spiral density waves," Astrophysical Journal Letters, vol. 715, no. 1, pp. L56-L61, 2010.

[195] R. Buta, D. L. Block, and J. H. Knapen, "A technique for separating the gravitational torques of bars and spirals in disk galaxies," The Astronomical Journal, vol. 126, no. 3, pp. 11481158, 2003.

[196] D. L. Block, R. Buta, J. H. Knapen, D. M. Elmegreen, B. G. Elmegreen, and I. Puerari, "Gravitational bar and spiral arm torques from Ks-band observations and implications for the pattern speeds," The Astronomical Journal, vol. 128, no. 1, pp. 183-201, 2004.

[197] R. G. Abraham and M. R. Merrifield, "Explorations in Hubble space: a quantitative tuning fork," The Astronomical Journal, vol. 120, no. 6, pp. 2835-2842, 2000.

[198] E. Laurikainen, H. Salo, and P. Rautiainen, "Comparison of bar strengths in active and non-active galaxies," Monthly Notices of the Royal Astronomical Society, vol. 331, no. 4, pp. 880-892, 2002.

[199] G. Contopoulos, "How far do bars extend," Astronomy and Astrophysics, vol. 81, no. 1-2, pp. 198-209, 1980.

[200] E. M. Corsini, "Direct measurement of bar pattern speeds," Memorie della Societa Astronomica Italiana Supplementi, vol. 18, p. 23, 2011.

[201] J. H. Hunter Jr., M. N. England, S. T. Gottesman, R. Ball, and J. M. Huntley Jr., "Dynamical models of the barred spiral galaxy NGC 3992," Astrophysical Journal, vol. 324, pp. 721740, 1988.

[202] M. N. England, S. T. Gottesman, and J. H. Hunter, "Highresolution observations, kinematics, and dynamics of the barred spiral NGC 1073," Astrophysical Journal Letters, vol. 348, no. 2, pp. 456-466, 1990.

[203] P. A. B. Lindblad, P. O. Lindblad, and E. Athanassoula, "Hydrodynamical simulations of the barred spiral galaxy NGC1365: dynamical interpretation of observations," Astronomy and Astrophysics, vol. 313, no. 1, pp. 65-90, 1996.

[204] P. A. B. Lindblad and H. Kristen, "Hydrodynamical simulations of the barred spiral galaxy NGC 1300: dynamical 
interpretation of observations," Astronomy and Astrophysics, vol. 313, no. 3, pp. 733-749, 1996.

[205] S. Laine and C. H. Heller, "A minor-merger model for NGC 7479," Monthly Notices of the Royal Astronomical Society, vol. 308, no. 2, pp. 557-568, 1999.

[206] B. J. Weiner, J. A. Sellwood, and T. B. Williams, "The disk and dark halo mass of the barred galaxy NGC 4123. II. Fluiddynamical models," Astrophysical Journal Letters, vol. 546, no. 2, pp. 931-951, 2001.

[207] J. A. L. Aguerri, J. H. Hunter, M. Prieto, A. M. Varela, S. T. Gottesman, and C. Muñoz-Tuñón, "An hydrodynamical model of the barred galaxy NGC 5850," Astronomy and Astrophysics, vol. 373, no. 3, pp. 786-795, 2001.

[208] P. Rautiainen, H. Salo, and E. Laurikainen, "Model-based pattern speed estimates for 38 barred galaxies," Monthly Notices of the Royal Astronomical Society, vol. 388, no. 4, pp. $1803-$ 1818, 2008.

[209] T. S. van Albada and R. H. Sanders, "Periodic orbits and gas flow in barred spirals," Monthly Notices of the Royal Astronomical Society, vol. 201, pp. 303-316, 1982.

[210] E. Athanassoula, "The existence and shapes of dust lanes in galactic bars," Monthly Notices of the Royal Astronomical Society, vol. 259, no. 2, pp. 345-364, 1992.

[211] S. Tremaine and M. D. Weinberg, "A kinematic method for measuring the pattern speed of barred galaxies," Astrophysical Journal, vol. 282, pp. L5-L7, 1984.

[212] S. M. Kent, "The pattern speed in the SB0 galaxy NGC 936," The Astronomical Journal, vol. 93, pp. 1062-1069, 1987.

[213] M. R. Merrifield and K. Kuijken, "The pattern speed of the bar in NGC 936," Monthly Notices of the Royal Astronomical Society, vol. 274, no. 3, pp. 933-938, 1995.

[214] J. Gerssen, K. Kuijken, and M. R. Merrifield, "The pattern speed of the bar in NGC 4596," Monthly Notices of the Royal Astronomical Society, vol. 306, no. 4, pp. 926-930, 1999.

[215] V. P. Debattista, E. M. Corsini, and J. A. L. Aguerri, "A fast bar in the post-interaction galaxy NGC 1023," Monthly Notices of the Royal Astronomical Society, vol. 332, no. 1, pp. 65-77, 2002.

[216] E. M. Corsini, J. A. L. Aguerri, V. P. Debattista, A. Pizzella, F. D. Barazza, and H. Jerjen, "The bar pattern speed of dwarf galaxy NGC 4431,” Astrophysical Journal, vol. 659, no. 2, pp. L121-L124, 2007.

[217] V. P. Debattista and J. A. Sellwood, "Dynamical friction and the distribution of dark matter in barred galaxies," Astrophysical Journal Letters, vol. 493, no. 1, pp. L5-L8, 1998.

[218] V. P. Debattista and J. A. Sellwood, "Constraints from dynamical friction on the dark matter content of barred galaxies," Astrophysical Journal, vol. 543, no. 2, pp. 704-721, 2000.

[219] E. Athanassoula, "What determines the strength and the slowdown rate of bars?" Monthly Notices of the Royal Astronomical Society, vol. 341, no. 4, pp. 1179-1198, 2003.

[220] F. D. Barazza, B. Binggeli, and H. Jerjen, "VLT surface photometry and isophotal analysis of early-type dwarf galaxies in the Virgo cluster," Astronomy and Astrophysics, vol. 407, no. 1, pp. 121-135, 2003.

[221] A. Sandage and B. Binggeli, "Studies of the Virgo cluster. III-a classification system and an illustrated atlas of Virgo cluster dwarf galaxies," The Astronomical Journal, vol. 89, pp. 919-931, 1984.

[222] B. Binggeli and C. C. Popescu, "Dwarf galaxies in the Virgo cluster. III. Flattening distributions," Astronomy and Astrophysics, vol. 298, p. 63, 1995.
[223] H. Jerjen, A. Kalnajs, and B. Binggeli, "IC3328: a "dwarf elliptical galaxy" with spiral structure," Astronomy and Astrophysics, vol. 358, no. 3, pp. 845-849, 2000.

[224] F. D. Barazza, B. Binggeli, and H. Jerjen, "More evidence for hidden spiral and bar features in bright early-type dwarf galaxies," Astronomy and Astrophysics, vol. 391, no. 3, pp. 823831, 2002.

[225] T. Lisker, E. K. Grebel, and B. Binggeli, "Virgo cluster earlytype dwarf galaxies with the sloan digital sky survey. I. On the possible disk nature of bright early-type dwarfs," The Astronomical Journal, vol. 132, no. 2, pp. 497-513, 2006.

[226] T. Lisker, K. Glatt, P. Westera, and E. K. Grebel, "Virgo cluster early-type dwarf galaxies with the sloan digital sky survey. II. Early-type dwarfs with central star formation," The Astronomical Journal, vol. 132, no. 6, pp. 2432-2452, 2006.

[227] T. Lisker, E. K. Grebel, B. Binggeli, and K. Glatt, "Virgo Cluster early-type dwarf galaxies with the sloan digital sky survey. III. Subpopulations: distributions, shapes, origins," Astrophysical Journal Letters, vol. 660, no. 2, pp. 1186-1197, 2007.

[228] T. Lisker, J. Janz, G. Hensler et al., "The first generation of virgo cluster dwarf elliptical galaxies?” Astrophysical Journal Letters, vol. 706, no. 1, pp. L124-L128, 2009.

[229] J. Kormendy, D. B. Fisher, M. E. Cornell, and R. Bender, "Structure and formation of elliptical and spheroidal galaxies," Astrophysical Journal, Supplement Series, vol. 182, no. 1, pp. 216-309, 2009.

[230] R. F. G. Wyse, G. Gilmore, and M. Franx, "Galactic bulges," Annual Review of Astronomy and Astrophysics, vol. 35, no. 1, pp. 637-675, 1997.

[231] S. Richard, C. B. Brook, H. Martel, D. Kawata, B. K. Gibson, and P. Sanchez-Blazquez, "Structure, kinematics and chemical enrichment patterns after major gas-rich disc-disc mergers," Monthly Notices of the Royal Astronomical Society, vol. 402, no. 3, pp. 1489-1503, 2010.

[232] T. J. Cox, S. N. Dutta, T. Di Matteo et al., "The kinematic structure of merger remnants," Astrophysical Journal, vol. 650, no. 2, pp. 791-811, 2006.

[233] J. E. Barnes, "Transformations of galaxies. I. Mergers of equae-mass stellar disks," Astrophysical Journal Letters, vol. 393, no. 2, pp. 484-507, 1992.

[234] L. Hernquist, "Structure of merger remnants. I. Bulgeless progenitors," Astrophysical Journal Letters, vol. 400, no. 2, pp. 460-475, 1992.

[235] V. Springel, "Modelling star formation and feedback in simulations of interacting galaxies," Monthly Notices of the Royal Astronomical Society, vol. 312, no. 4, pp. 859-879, 2000.

[236] A. C. González-García and M. Balcells, "Elliptical galaxies from mergers of discs," Monthly Notices of the Royal Astronomical Society, vol. 357, no. 2, pp. 753-772, 2005.

[237] T. Naab and I. Trujillo, "Surface density profiles of collisionless disc merger remnants," Monthly Notices of the Royal Astronomical Society, vol. 369, no. 2, pp. 625-644, 2006.

[238] P. F. Hopkins, K. Bundy, D. Croton et al., "Mergers and bulge formation in $\lambda \mathrm{cDM}$ : which mergers matter?" Astrophysical Journal Letters, vol. 715, no. 1, pp. 202-229, 2010.

[239] B. Robertson, T. J. Cox, L. Hernquist et al., "The fundamental scaling relations of elliptical galaxies," The Astrophysical Journal, vol. 641, no. 1, pp. 21-40, 2006.

[240] S. P. Bamford, R. C. Nichol, I. K. Baldry et al., "Galaxy Zoo: the dependence of morphology and colour on environment," Monthly Notices of the Royal Astronomical Society, vol. 393, no. 4, pp. 1324-1352, 2009. 
[241] S. J. Kannappan, J. M. Guie, and A. J. Baker, "E/S0 galaxies on the blue color-stellar mass sequence at $\mathrm{z}=0$ : fading mergers or future spirals?" The Astronomical Journal, vol. 138, no. 2, pp. 579-597, 2009.

[242] K. Schawinski, C. Lintott, and D. Thomas, "Galaxy Zoo: a sample of blue early-type galaxies at low redshift," Monthly Notices of the Royal Astronomical Society, vol. 396, pp. 818829.

[243] J. A. L. Aguerri, M. Balcells, and R. F. Peletier, "Growth of galactic bulges by mergers. I. Dense satellites," Astronomy and Astrophysics, vol. 367, no. 2, pp. 428-442, 2001.

[244] G. J. Bendo and J. E. Barnes, "The line-of-sight velocity distributions of simulated merger remnants," Monthly Notices of the Royal Astronomical Society, vol. 316, no. 2, pp. 315-325, 2000.

[245] N. Cretton, T. Naab, H. W. Rix, and A. Burkert, "The kinematics of 3:1 merger remnants and the formation of lowluminosity elliptical galaxies," Astrophysical Journal Letters, vol. 554, no. 1, pp. 291-297, 2001.

[246] G. Toth and J. P. Ostriker, "Galactic disks, infall, and the global value of Omega," Astrophysical Journal, vol. 389, pp. 5-26, 1992.

[247] D. A. Quin, J. G. Doyle, C. J. Butler, P. B. Byrne, and J. H. Swank, "Rotational modulation and flares on Rs-Canum and by-draconis stars-part seventeen-ultraviolet spectroscopy and optical photometry of Au-microscopii in 1986," Astronomy and Astrophysics, vol. 272, no. 2, p. 477, 1993.

[248] I. R. Walker, J. Christopher Mihos, and L. Hernquist, "Quantifying the fragility of galactic disks in minor mergers," Astrophysical Journal Letters, vol. 460, no. 1, pp. 121-135, 1996.

[249] J. C. Mihos, "Morphology of galaxy mergers at intermediate redshift," Astrophysical Journal, vol. 438, pp. L75-L78, 1995.

[250] M. C. Eliche-Moral, M. Balcells, J. A. L. Aguerri, and A. C. González-García, "Growth of galactic bulges by mergers II. Low-density satellites," Astronomy and Astrophysics, vol. 457, no. 1, pp. 91-108, 2006.

[251] M. C. Eliche-Moral, A. C. Gonzalez-Garcia, M. Balcells et al., "A minor merger origin for stellar inner discs and rings in spiral galaxies," Astronomy and Astrophysics, vol. 533, article A104, 2011.

[252] K. Bekki, "Unequal-mass galaxy mergers and the creation of cluster S0 galaxies," Astrophysical Journal Letters, vol. 502, p. L133, 1998.

[253] F. Bournaud, C. J. Jog, and F. Combes, "Galaxy mergers with various mass ratios: properties of remnants," Astronomy and Astrophysics, vol. 437, no. 1, pp. 69-85, 2005.

[254] D. Christlein and A. I. Zabludoff, "Can early-type galaxies evolve from the fading of the disks of late-type galaxies?" The Astrophysical Journal, vol. 616, pp. 192-198, 2004.

[255] J. R. Hargis, K. L. Rhode, J. Strader, and J. P. Brodie, "The globular cluster population of NGC 7457: clues to the evolution of field S0 galaxies," The Astrophysical Journal, vol. 738, no. 1, p. 113, 2011.

[256] F. Prada, C. M. Gutierrez, R. F. Peletier, and C. D. McKeith, "A counterrotating bulge in the S(b) galaxy NGC 7331," Astrophysical Journal Letters, vol. 463, p. L9, 1996.

[257] S. Smith, "Some notes on the structure of elliptical nebulae," Astrophysical Journal, vol. 82, p. 192, 1935.

[258] F. Zwicky, "Clusters of nebulae," Publications of the Astronomical Society of the Pacific, vol. 54, no. 320, p. 185, 1942.

[259] A. R. Sandage, "Review of publications- (the hubble atlas of galaxies)," Journal of the Royal Astronomical Society of Canada, vol. 56, p. 29, 1962.
[260] B. Moore, N. Katz, G. Lake, A. Dressler, and A. Oemler, "Galaxy harassment and the evolution of clusters of galaxies," Nature, vol. 379, no. 6566, pp. 613-616, 1996.

[261] A. Oemler Jr., "The systematic properties of clusters of galaxies. Photometry of 15 clusters," Astrophysical Journal, vol. 194, pp. 1-20, 1974.

[262] A. Dressler, "Galaxy morphology in rich clusters-implications for the formation and evolution of galaxies," Astrophysical Journal, vol. 236, pp. 351-365, 1980.

[263] A. Dressler, A. Oemler Jr., W. J. Couch et al., "Evolution since $\mathrm{Z}=0.5$ of the morphology-density relation for clusters of galaxies," Astrophysical Journal, vol. 490, p. 577, 1997.

[264] G. Fasano, B. M. Poggianti, W. J. Couch, D. Bettoni, P. Kjærgaard, and M. Moles, "The evolution of the galactic morphological types in clusters," Astrophysical Journal, vol. 542, no. 2, pp. 673-683, 2000.

[265] J. E. Gunn and J. R. Gott III, "On the infall of matter into clusters of galaxies and some effects on their evolution," Astrophysical Journal, vol. 176, p. 1, 1972.

[266] V. Quilis, B. Moore, and R. Bower, "Gone with the wind: the origin of SO galaxies in clusters," Science, vol. 288, no. 5471, pp. 1617-1620, 2000.

[267] K. Bekki, "Ram-pressure stripping of halo gas in disc galaxies: implications for galactic star formation in different environments," Monthly Notices of the Royal Astronomical Society, vol. 399, no. 4, pp. 2221-2230, 2009.

[268] A. Toomre and J. Toomre, "Galactic bridges and tails," Astrophysical Journal, vol. 178, pp. 623-666, 1972.

[269] K. Bekki, "Galaxy mergers and the creation of cluster S0 galaxies," Astrophysics and Space Science, vol. 276, no. 2-4, pp. 847-850, 2001.

[270] K. Bekki, W. J. Couch, and Y. Shioya, "Passive spiral formation from halo gas starvation: gradual transformation into S0s," Astrophysical Journal Letters, vol. 577, no. 2, pp. 651657, 2002.

[271] I. G. McCarthy, C. S. Frenk, A. S. Font et al., "Ram pressure stripping the hot gaseous halos of galaxies in groups and clusters," Monthly Notices of the Royal Astronomical Society, vol. 383, no. 2, pp. 593-605, 2008.

[272] D. Kawata and J. S. Mulchaey, "Strangulation in galaxy groups," Astrophysical Journal Letters, vol. 672, no. 2, pp. L103-L106, 2008.

[273] J. D. P. Kenney, J. H. Van Gorkom, and B. Vollmer, "VLA H I observations of gas stripping in the Virgo Cluster spiral NGC 4522," The Astronomical Journal, vol. 127, no. 6, pp. 33613374, 2004.

[274] H. H. Crowl, J. D. P. Kenney, J. H. Van Gorkom, and B. Vollmer, "Dense cloud ablation and ram pressure stripping of the Virgo spiral NGC 4402," The Astronomical Journal, vol. 130, no. 1, pp. 65-72, 2005.

[275] B. Vollmer, M. Soida, K. Otmianowska-Mazur, J. D. P. Kenney, J. H. Van Gorkom, and R. Beck, "A dynamical model for the heavily ram pressure stripped Virgo spiral galaxy NGC 4522," Astronomy and Astrophysics, vol. 453, no. 3, pp. 883893, 2006.

[276] B. Vollmer, W. Huchtmeier, and W. Van Driel, "NGC 4254: a spiral galaxy entering the Virgo cluster," Astronomy and Astrophysics, vol. 439, no. 3, pp. 921-933, 2005.

[277] B. Vollmer, J. Braine, C. Pappalardo, and P. Hily-Blant, "Rampressure stripped molecular gas in the Virgo spiral galaxy NGC 4522," Astronomy and Astrophysics, vol. 491, no. 2, pp. 455-464, 2008.

[278] B. Vollmer, M. Soida, A. Chung et al., "Ram pressure stripping of the multiphase ISM in the Virgo cluster spiral galaxy 
NGC 4438," Astronomy and Astrophysics, vol. 496, no. 3, pp. 669-675, 2009.

[279] A. Abramson, J. D. P. Kenney, H. H. Crowl et al., "Caught in the act: strong, active ram pressure stripping in virgo cluster spiral NGC4330," The Astronomical Journal, vol. 141, no. 5, article 164, 2011.

[280] V. Cayatte, J. H. Van Gorkom, C. Balkowski, and C. Kotanyi, "VLA observations of neutral hydrogen in Virgo Cluster galaxies. I. The atlas," The Astronomical Journal, vol. 100, no. 3, pp. 604-634, 1990.

[281] M. P. Haynes, R. Giovanelli, and G. L. Chincarini, "The influence of envirionment on the H I content of galaxies," Annual Review of Astronomy and Astrophysics, vol. 22, pp. 445-470, 1984.

[282] J. M. Solanes, A. Manrique, C. García-Gómez et al., “The H I content of spirals. II. Gas deficiency in cluster galaxies," The Astrophysical Journal, vol. 548, no. 1, pp. 97-113, 2001.

[283] B. R. Kent, R. Giovanelli, M. P. Haynes et al., "The Arecibo legacy fast alfa survey. VI. Second HI source catalog of the Virgo cluster region," The Astronomical Journal, vol. 136, no. 2, pp. 713-724, 2008.

[284] A. Chung, J. H. van Gorkom, J. D. P. Kenney, H. Crowl, and B. Vollmer, "VLA imaging of virgo spirals in atomic gas (VIVA). I. The atlas and the H I properties," The Astronomical Journal, vol. 138, no. 6, p. 1741, 2009.

[285] J. M. Barr, A. G. Bedregal, A. Aragón-Salamanca, M. R. Merrifield, and S. P. Bamford, "The formation of S0 galaxies: evidence from globular clusters," Astronomy and Astrophysics, vol. 470, no. 1, pp. 173-178, 2007.

[286] L. Mayer, F. Governato, M. Colpi et al., "Tidal stirring and the origin of dwarf spheroidals in the local group," Astrophysical Journal Letters, vol. 547, no. 2, pp. L123-L127, 2001.

[287] L. Mayer, B. Moore, T. Quinn, F. Governato, and J. Stadel, "Tidal debris of dwarf spheroidals as a probe of structure formation models," Monthly Notices of the Royal Astronomical Society, vol. 336, no. 1, pp. 119-130, 2002.

[288] C. Mastropietro, B. Moore, L. Mayer, V. P. Debattista, R. Piffaretti, and J. Stadel, "Morphological evolution of discs in clusters," Monthly Notices of the Royal Astronomical Society, vol. 364, no. 2, pp. 607-619, 2005.

[289] J. A. L. Aguerri and A. C. González-García, "On the origin of dwarf elliptical galaxies: the fundamental plane," Astronomy and Astrophysics, vol. 494, no. 3, pp. 891-904, 2009.

[290] S. Kazantzidis, E. L. Lokas, S. Callegari, L. Mayer, and L. A. Moustakas, "On the efficiency of the tidal stirring mechanism for the origin of dwarf spheroidals: dependence on the orbital and structural parameters of the progenitor disky dwarfs," The Astrophysical Journal, vol. 726, no. 2, article 98, 2011.

[291] M. Arnaboldi, J. A. L. Aguerri, N. R. Napolitano et al., "Intracluster planetary nebulae in Virgo: photometric selection, spectroscopic validation, and cluster depth," The Astronomical Journal, vol. 123, no. 2, pp. 760-771, 2002.

[292] M. Arnaboldi, O. Gerhard, J. A. L. Aguerri et al., "The line-ofsight velocity distributions of intracluster planetary nebulae in the virgo cluster core," Astrophysical Journal, vol. 614, no. 1, pp. L33-L36, 2004.

[293] J. J. Feldmeier, R. Ciardullo, G. H. Jacoby, and P. R. Durrell, "Intracluster planetary nebulae in the Virgo Cluster. III. Luminosity of the intracluster light and tests of the spatial distribution," Astrophysical Journal, vol. 615, no. 1 I, pp. 196208, 2004.

[294] J. A. L. Aguerri, O. E. Gerhard, M. Arnaboldi, N. R. Napolitano, N. Castro-Rodriguez, and K. C. Freeman, "Intracluster stars in the Virgo Cluster core," The Astronomical Journal, vol. 129, no. 6, pp. 2585-2596, 2005.

[295] B. F. Williams, R. Ciardullo, P. R. Durrell et al., "Virgo's intracluster globular clusters as seen by the advanced camera for surveys," The Astrophysical Journal, vol. 654, no. 2, pp. 835-843, 2007.

[296] N. Castro-Rodriguéz, M. Arnaboldi, J. A. L. Aguerri et al., "Intracluster light in the Virgo cluster: large scale distribution," Astronomy and Astrophysics, vol. 507, no. 2, pp. 621634, 2009.

[297] O. Gerhard, M. Arnaboldi, K. C. Freeman, N. Kashikawa, S. Okamura, and N. Yasuda, "Detection of intracluster planetary nebulae in the Coma Cluster," Astrophysical Journal Letters, vol. 621, no. 2, pp. L93-L96, 2005.

[298] O. Gerhard, M. Arnaboldi, K. C. Freeman, S. Okamura, N. Kashikawa, and N. Yasuda, "The kinematics of intracluster planetary nebulae and the on-going subcluster merger in the Coma cluster core," Astronomy and Astrophysics, vol. 468, no. 3, pp. 815-822, 2007.

[299] T. Theuns and S. J. Warren, "Intergalactic stars in the Fornax cluster," Monthly Notices of the Royal Astronomical Society, vol. 284, no. 3, pp. L11-L14, 1997.

[300] R. Sánchez-Janssen, J. Méndez-Abreu, and J. A. L. Aguerri, "Thin discs, thick dwarfs and the effects of stellar feedback," Monthly Notices of the Royal Astronomical Society, vol. 406, no. 1, pp. L65-L69, 2010.

[301] C. A. Tremonti, T. M. Heckman, G. Kaumann et al., "The origin of the mass-metallicity relation: insights from 53,000 star-forming galaxies in the sloan digital sky survey," The Astrophysical Journal, vol. 613, no. 2, pp. 898-913, 2004.

[302] I. Chilingarian, V. Cayatte, Y. Revaz et al., "A population of compact elliptical galaxies detected with the virtual observatory," Science, vol. 326, no. 5958, pp. 1379-1382, 2009.

[303] G. Byrd and M. Valtonen, "Tidal generation of active spirals and S0 galaxies by rich clusters," Astrophysical Journal Letters, vol. 350, no. 1, pp. 89-94, 1990.

[304] M. Valluri, "Compressive tidal heating of a disk galaxy in a rich cluster," Astrophysical Journal, vol. 408, no. 1, pp. 57-70, 1993.

[305] D. Merritt, "Relaxation and tidal stripping in rich clusters of galaxies. I. Evolution of the mass distribution," Astrophysical Journal, vol. 264, pp. 24-48, 1983.

[306] C. S. Frenk, A. E. Evrard, S. D. M. White, and F. J. Summers, "Galaxy dynamics in clusters," The Astrophysical Journal, vol. 472, no. 2, pp. 460-484, 1996.

[307] O. Y. Gnedin, "Tidal effects in clusters of galaxies," Astrophysical Journal Letters, vol. 582, no. 1, pp. 141-161, 2003.

[308] B. M. Poggianti, T. J. Bridges, D. Carter et al., "Ages of S0 and elliptical galaxies in the Coma Cluster," The Astrophysical Journal, vol. 563, no. 1, pp. 118-123, 2001.

[309] B. M. Poggianti, G. Fasano, D. Bettoni et al., "The evolution of spiral, S0, and elliptical galaxies in clusters," Astrophysical Journal Letters, vol. 697, no. 2, pp. L137-L140, 2009.

[310] W. Forman, C. Jones, L. David, and W. Tucker, "Observations and evolution of hot coronae around early type galaxies," Advances in Space Research, vol. 10, no. 2, pp. 217-220, 1990.

[311] C. Jones and W. Forman, "Einstein observatory images of clusters of galaxies," The Astrophysical Journal, vol. 511, no. 1, pp. 65-83, 1999.

[312] R. De Propris, M. Colless, J. A. Peacock et al., "The 2dF Galaxy Redshift Survey: the blue galaxy fraction and implications for the Butcher-Oemler effect," Monthly Notices of the Royal Astronomical Society, vol. 351, no. 1, pp. 125-132, 2004. 
[313] Y.-S. Li and A. Helmi, "Infall of substructures on to a Milky Way-like dark halo," Monthly Notices of the Royal Astronomical Society, vol. 385, no. 3, pp. 1365-1373, 2008.

[314] J. C. Berrier, K. R. Stewart, J. S. Bullock, C. W. Purcell, E. J. Barton, and R. H. Wechsler, "The assembly of galaxy clusters," Astrophysical Journal Letters, vol. 690, no. 2, pp. 1292-1302, 2009.

[315] J. A. L. Aguerri and R. Sánchez-Janssen, "A study of catalogued nearby galaxy clusters in the SDSS-DR4. II. Cluster substructure," Astronomy and Astrophysics, vol. 521, article A28, 2010.

[316] K. Bekki, D. A. Forbes, M. A. Beasley, and W. J. Couch, "Globular cluster formation from gravitational tidal effects of merging and interacting galaxies," Monthly Notices of the Royal Astronomical Society, vol. 335, no. 4, pp. 1176-1192, 2002.

[317] N. Caldwell, J. A. Rose, R. M. Sharples, R. S. Ellis, and R. G. Bower, "Star formation in early-type galaxies in the coma cluster," The Astronomical Journal, vol. 106, no. 2, pp. 473492, 1993.

[318] N. Caldwell and J. A. Rose, "The Butcher-Oemler effect at low redshift: spectroscopy of five nearby clusters of galaxies," The Astronomical Journal, vol. 113, no. 2, pp. 492-520, 1997.

[319] K. Bekki, "Group-cluster merging and the formation of starburst galaxies," Astrophysical Journal Letters, vol. 510, no. 1, pp. L15-L19, 1999.

[320] D. Clowe, A. Gonzalez, and M. Markevitch, "Weak-lensing mass reconstruction of the interacting cluster 1E 0657-558: direct evidence for the existence of dark matter," Astrophysical Journal, vol. 604, no. 2, pp. 596-603, 2004.

[321] D. Clowe, P. Schneider, A. Aragon-Salamanca et al., "Weak lensing mass reconstructions of the ESO Distant Cluster Survey," Astronomy and Astrophysics, vol. 451, no. 2, pp. 395408, 2006

[322] A. Mahdavi, H. Hoekstra, A. Babul, D. D. Balam, and P. L. Capak, "A dark core in Abell 520," Astrophysical Journal Letters, vol. 668, no. 2, pp. 806-814, 2007.

[323] M. Bradač, S. W. Allen, T. Treu et al., "Revealing the properties of dark matter in the merging cluster MACS J0025.4-1222," The Astrophysical Journal, vol. 687, no. 2, pp. 959-967, 2008.

[324] C. J. Ma, H. Ebeling, P. Marshall, and T. Schrabback, "The impact of a major cluster merger on galaxy evolution in MACS J0025.4-1225," Monthly Notices of the Royal Astronomical Society, vol. 406, no. 1, pp. 121-136, 2010.

[325] K. Bundy, R. S. Ellis, and C. J. Conselice, "The mass assembly histories of galaxies of various morphologies in the goods fields," Astrophysical Journal, vol. 625, no. 2, pp. 621-632, 2005.

[326] A. Cimatti, E. Daddi, and A. Renzini, "Mass downsizing and "top-down" assembly of early-type galaxies," Astronomy and Astrophysics, vol. 453, no. 2, pp. L29-L33, 2006.

[327] A. Dekel and J. Silk, "The origin of dwarf galaxies, cold dark matter, and biased galaxy formation," Astrophysical Journal, vol. 303, pp. 39-55, 1986.

[328] J. Silk and M. J. Rees, "Quasars and galaxy formation," Astronomy and Astrophysics, vol. 331, pp. L1-L4, 1998.

[329] L. Ciotti and J. P. Ostriker, "Cooling flows and quasars: different aspects of the same phenomenon? I. Concepts," Astrophysical Journal Letters, vol. 487, no. 2, pp. L105-L108, 1997.

[330] V. Springel, T. Di Matteo, and L. Hernquist, "Modelling feedback from stars and black holes in galaxy mergers," Monthly Notices of the Royal Astronomical Society, vol. 361, no. 3, pp. 776-794, 2005.
[331] J. Silk, "Ultraluminous starbursts from supermassive black hole-induced outflows," Monthly Notices of the Royal Astronomical Society, vol. 364, no. 4, pp. 1337-1342, 2005.

[332] K. Schawinski, S. Khochfar, S. Kaviraj et al., "Suppression of star formation in early-type galaxies by feedback from supermassive black holes," Nature, vol. 442, no. 7105, pp. 888-891, 2006.

[333] K. Schawinski, D. Thomas, M. Sarzi et al., "Observational evidence for AGN feedback in early-type galaxies," Monthly Notices of the Royal Astronomical Society, vol. 382, no. 4, pp. 1415-1431, 2007.

[334] K. L. Masters, M. Mosleh, and A. K. Romer, "Galaxy Zoo: passive red spirals," Monthly Notices of the Royal Astronomical Society, vol. 405, pp. 783-799, 2010.

[335] C. Wolf, A. Aragon-Salamanca, M. Balogh et al., "The STAGES view of red spirals and dusty red galaxies: massdependent quenching of star formation in cluster infall," Monthly Notices of the Royal Astronomical Society, vol. 393, no. 4, pp. 1302-1323, 2009. 

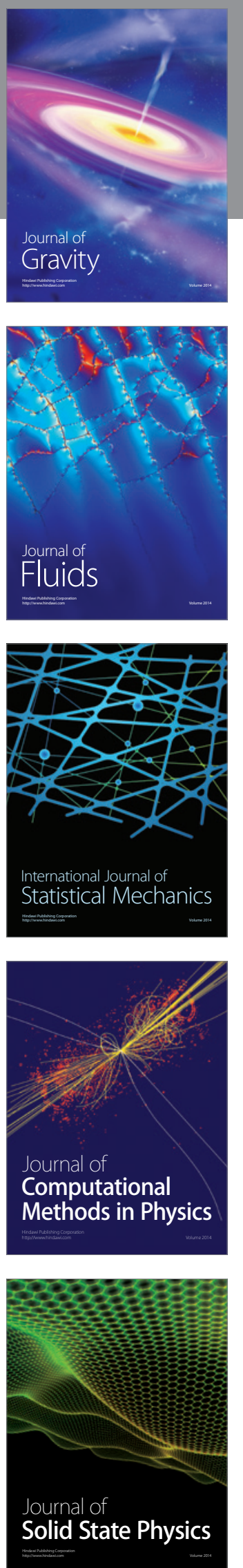

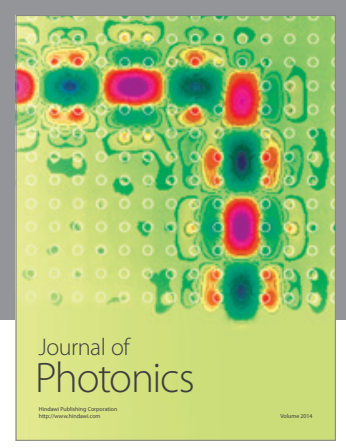

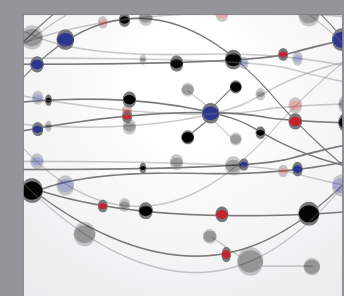

The Scientific World Journal
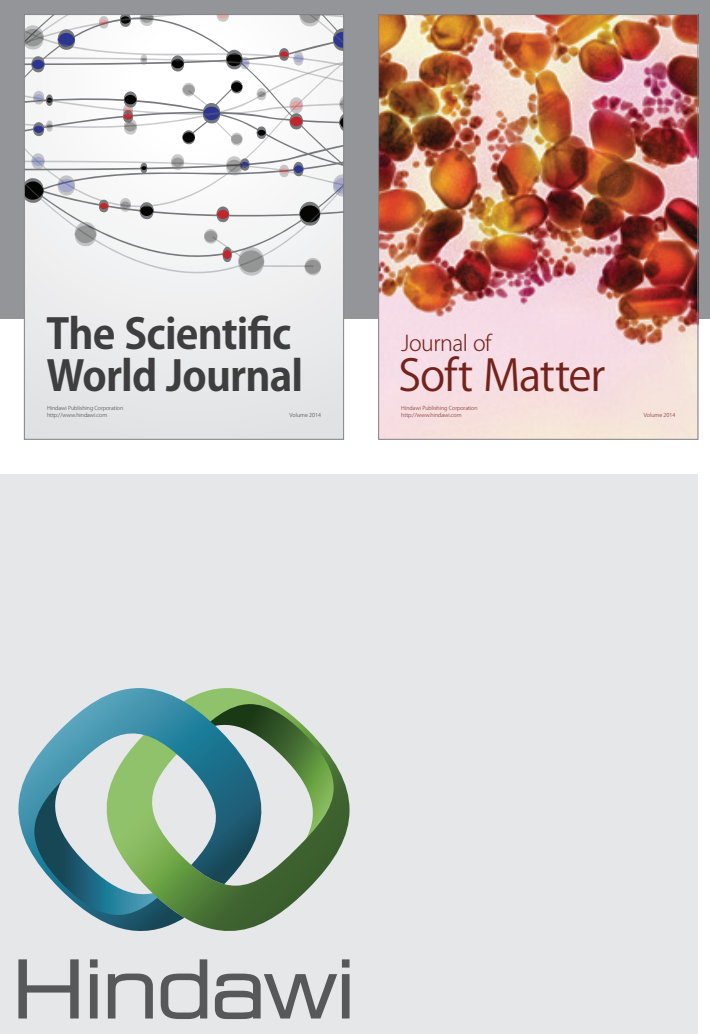

Submit your manuscripts at

http://www.hindawi.com
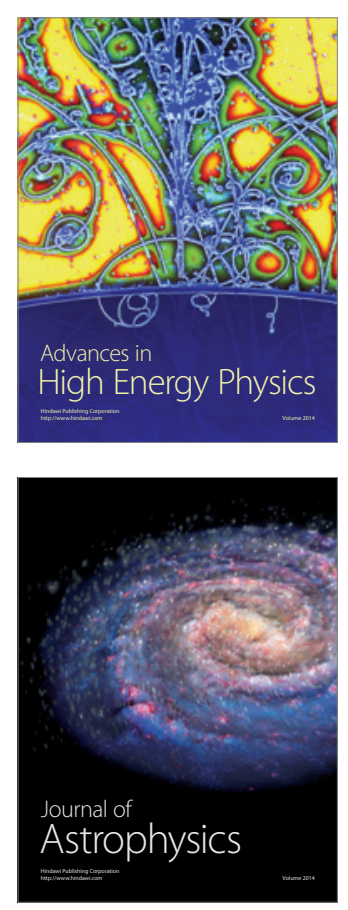
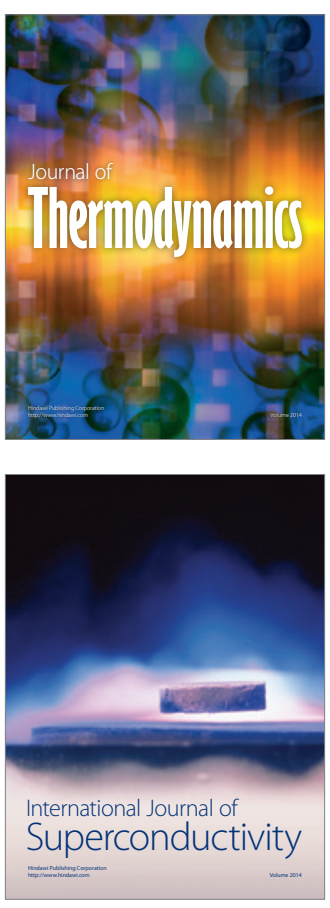
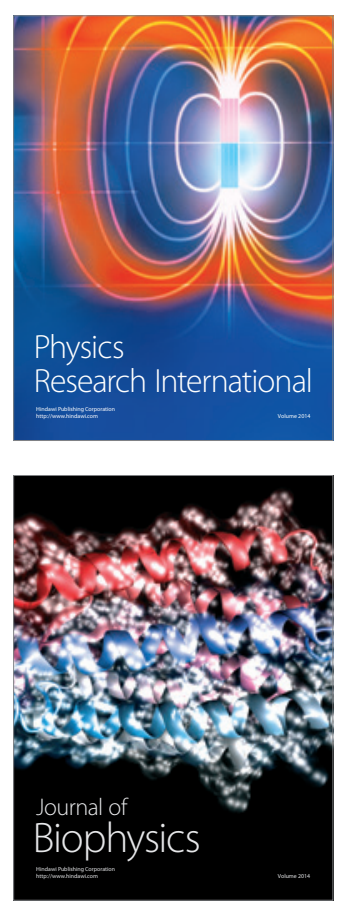
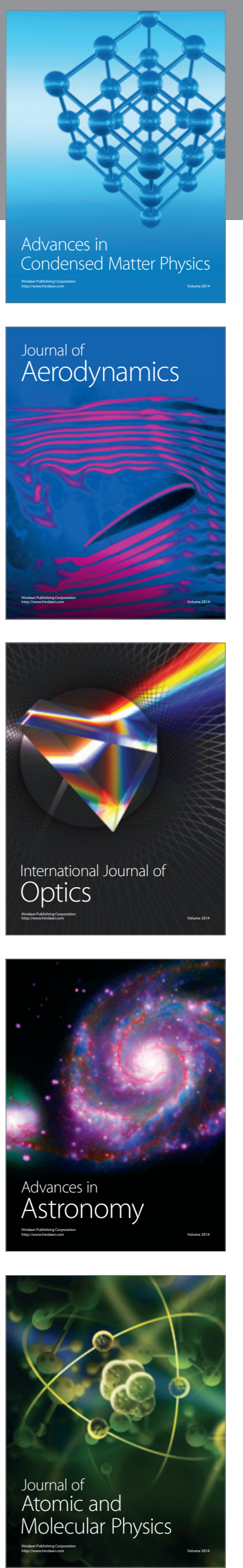